\title{
Energetic Deposition of Niobium Thin Film in Vacuum
}

\author{
Genfa Wu
}

\author{
Dissertation submitted to the Faculty of the \\ Virginia Polytechnic Institute and State University \\ in partial fulfillment of the requirements for the degree of \\ Doctor of Philosophy \\ in \\ Physics
}

H. Lawrence Phillips, Co-Chair

John Ficenec, Chair

James R. Heflin

David Jenkins

Ronald M. Sundelin

Blacksburg, Virginia, June 14, 2002

Keywords: Thin Film Deposition, Energetic Condensation, ECR plasma, Niobium, RF superconductivity, Particle Accelerator.

Copyright 2002, Genfa Wu 


\title{
Energetic Deposition of Niobium Thin Film in Vacuum
}

\author{
Genfa Wu
}

\section{(Abstract)}

Niobium thin films are expected to be free of solid inclusions commonly seen in solid niobium. For particle accelerators, niobium thin film has the potential to replace the solid niobium in the making of the accelerating structures. In order to understand and improve the superconducting performance of niobium thin films at cryogenic temperature, an energetic vacuum deposition system has been developed to study deposition energy effects on the properties of niobium thin films on various substrates. The system directly uses microwave power to create a pure niobium plasma, which can be used to extract niobium ion flux with controllable kinetic energy for direct deposition. The ultra high vacuum avoids the gaseous inclusions in thin films. A retarding field energy analyzer is developed and used to measure the kinetic energy of niobium at the substrate location. A systematic process for thin film characterization is developed and used to analyze the niobium thin films made by this energetic condensation. The properties of niobium thin films at several deposition energies are obtained, and the results show that there exists a preferred deposition energy around $115 \mathrm{eV}$. 
Acknowledgement

\section{Acknowledgement}

Prof. Ron Sundelin has supported me through out the dissertation work at Jefferson Lab. His guidance and help extended beyond the scientific scope, and probably will influence through out my lifetime. Dr. Larry Phillips, as my day-to-day supervisor, looked after many technical details during the system development. Many times, Larry helped to expedite the work requests and material acquisitions. Both Ron and Larry's extensive scientific knowledge made my student life enviable by others. Beside their mentorship, Ron and Larry are also the best friends I can have. They helped me in many ways to settle into the American cultures. Julie Oyer and Chris Thomas helped me in every administrative detail, in a way that I felt "spoiled". I am grateful for their help that I can focus on my dissertation.

I also got encouragement from other committee members like Prof. David Jenkins, Prof. John Ficenec, Prof. Luke Mo and Prof. Randy Heflin. Prof. John Ficenec provided many writing suggestions.

Many staff members at Jefferson Lab are supportive and helpful. Dr. Peter Kneisel and John Musson are first two staff I want to express lot of appreciations. Their support and encouragement were countless and critical for my dissertation work. Charlie Reece, Larry Doolittle and Ganapati Myneni are also supportive and shared their scientific knowledge. Viet Nguyen helped me with HFSS program. Sam Morgan, Brett Lewis and Peter Kushnick offered lot of help in machining, instrumentation and cryogenic testing. Ralph Afanador, Jennifer Williams and Tom Elliot helped me a lot about chemical cleaning and processes. Richard Bundy and John Brawley helped me with the e-beam welding. Help and valuable discussions also came from Andy Wu, Anne-Marie Valente, Tom Hiatt, John Mammosser, Joe Preble, Sherry Thomas, Audrey Nichols, Kim Laracuente, Trent Allison, Mark Wissman, Tom Goodman, Jody Brock, John Fischer, Jay Wall, Scott Williams, Ken Worland, Bob Bennett, Gary Slack, Bob Manos, Tim Rothgeb, Frank Folsom, Frank Humphry, Don Bullard and Michael Necaise.

Tong Wang helped me with the SEM and Field Emission Test. Paul Boccard offered many different kinds of help during my first year at Jefferson Lab. Christina Kraschle also helped during first year, and helped to proof read this whole report.

Material analysis support also came from Prof. Lou Hand, John Hunt, Maura Weathers at Cornell University, Brandt Robertson and Amy Wilkerson at Applied Research Center at Newport News. Hasan Padamsee at Cornell University helped Tc comparative measurement.

This work made use of the Cornell Center for Materials Research Shared Experimental Facilities, supported through the National Science Foundation Research Science and Engineering Centers program (DMR-9632275).

Both of my families here and oversea are very supportive. I thank them for their understanding and patience. 


\section{Content}

Chapter 1. Thin Film Cavities for Superconducting Radio Frequency Particle Accelerators ....................................

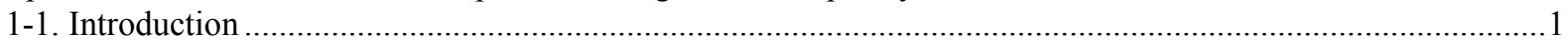

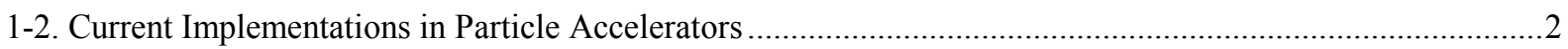

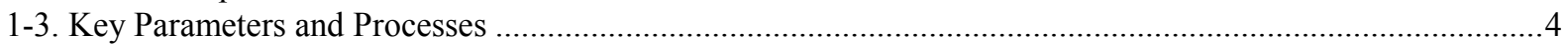

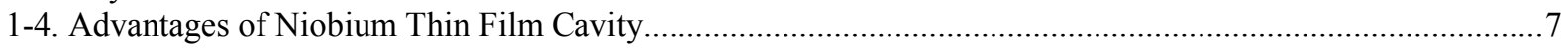

Chapter 2. The Comparison between the Existing Coating Techniques and the Energetic Condensation................10

2-1. Sputtering niobium thin film on copper...................................................................................... 10

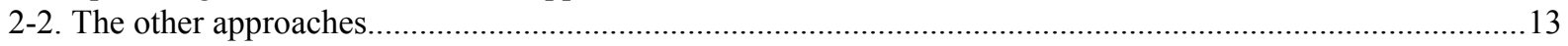

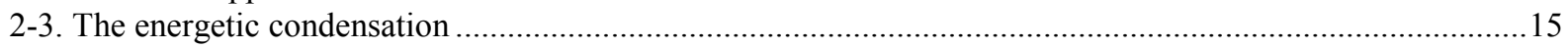

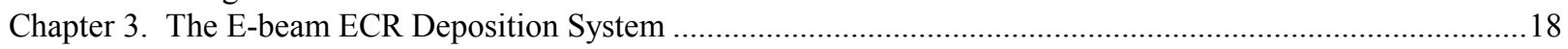

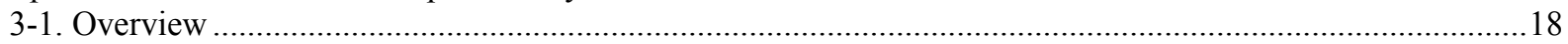

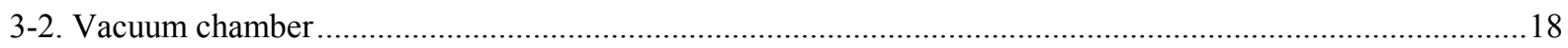

3-3. The principle of electron cyclotron resonance

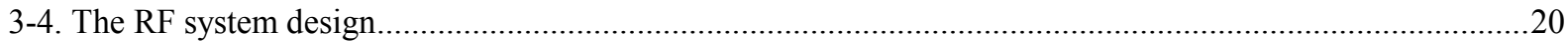

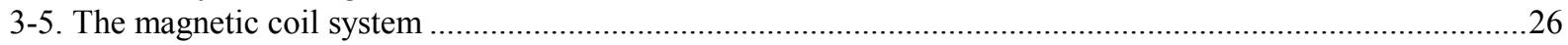

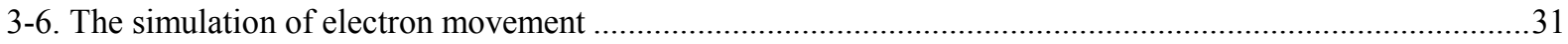

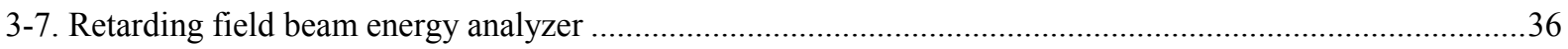

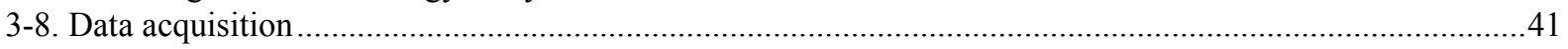

Chapter 4. Experiment with Argon, Niobium Vapor and Copper Vapor ............................................................42

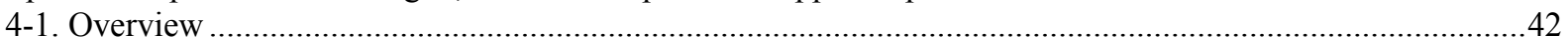

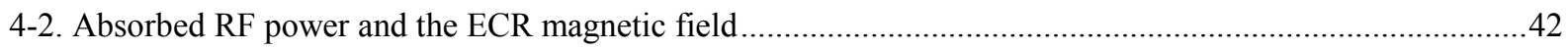

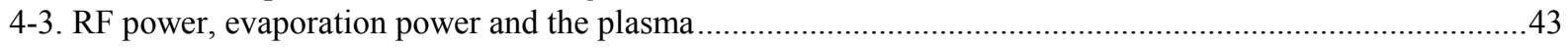

4-4. Measurement of niobium ion energy at the biased substrate …….............................................................

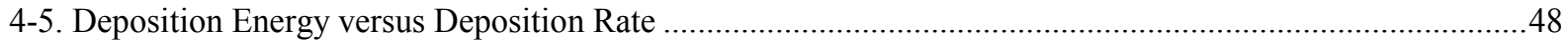

Chapter 5. The Characterization Procedures of Niobium Thin Film ...................................................................50

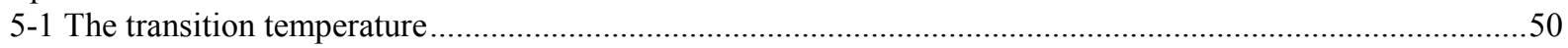

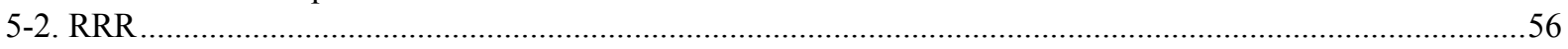

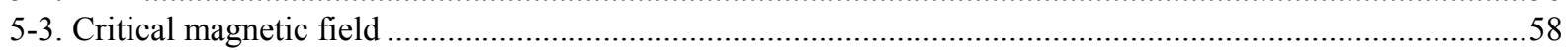

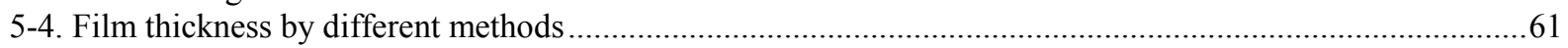

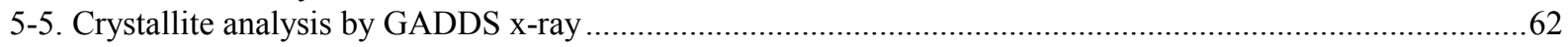

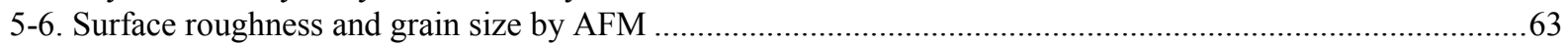

5-7. The surface quality check by Optical microscope and SEM ……..............................................................64

Chapter 6. Niobium Thin Film Deposition and the Properties at Different Deposition Energies ...........................65

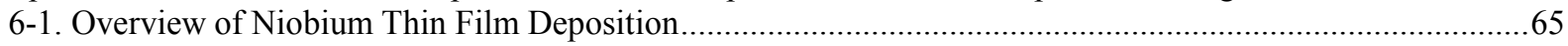

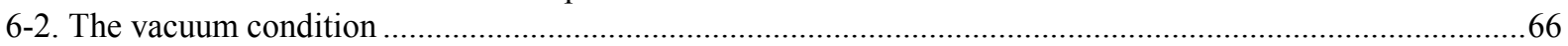

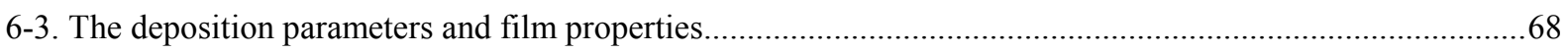

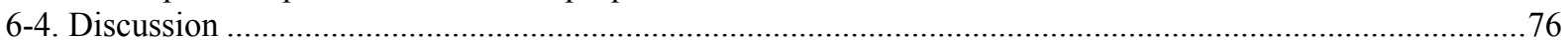

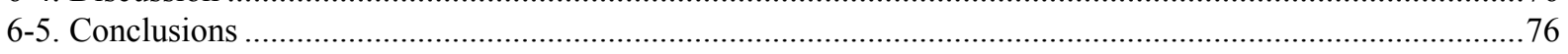

Appendix A. RGA Monitoring during One Deposition Run........................................................................... 77

Appendix B. The simulation code of electron movement inside the ECR RF field ........................................ 81

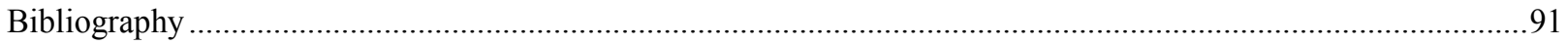




\section{List of Figures}

Fig. 1-1 A coarse model for temperature estimation around a $50 \mu \mathrm{m}$ size defect.............................................

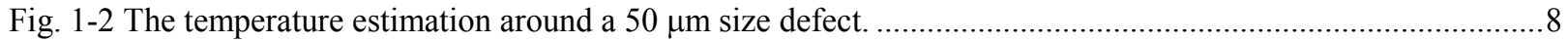

Fig. 2-1 The Q-degradation Seen on Sputtering-coated Nb Copper Cavity....................................................11

Fig. 2-2 Movchan-Demchishin Zone Model........................................................................................ 15

Fig. 2-3 The illustration of the surface atom nucleation process to show the columnar forming and atom mobility

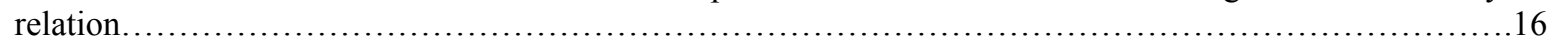

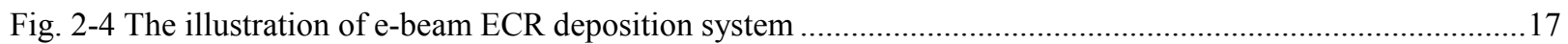

Fig. 3-1 Illustration of E-beam-ECR vacuum system ............................................................................ 19

Fig. 3-2 Electron being accelerated clock wisely by periodic electric field ................................................20

Fig. 3-3 Electron's spiral path in external magnetic field superimposed with perpendicular periodic electric field..20

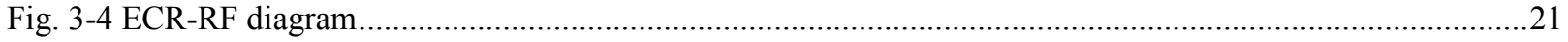

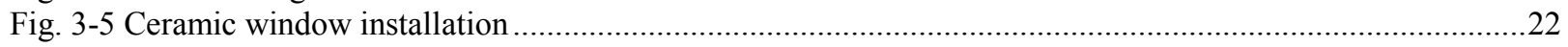

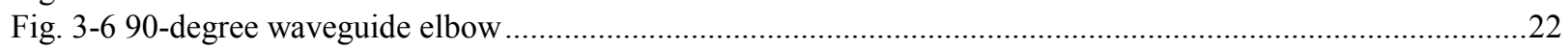

Fig. 3-7 Simulation models with two or three slots to reduce the RF radiation. ................................................24

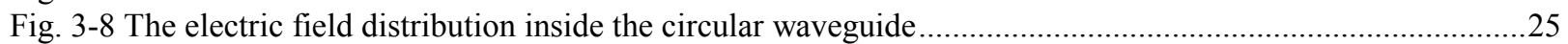

Fig. 3-9 The assembly views of ECR plasma chamber, the slots are not shown in the bottom open hole. ...............25

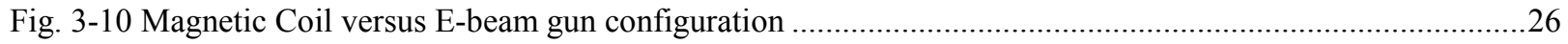

Fig. 3-11 Magnetic Coil field contour simulated by Pandira code, half of the coil cross section is shown. .............27

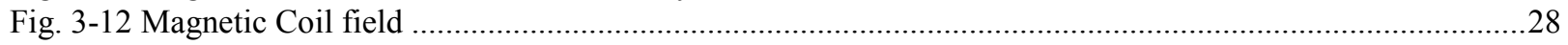

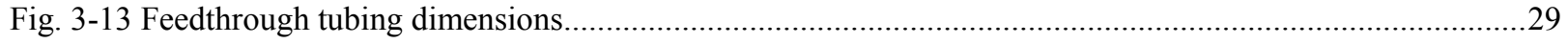

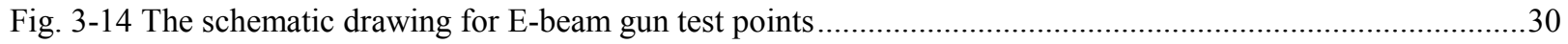

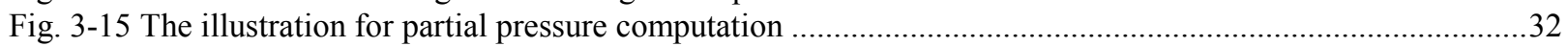

Fig. 3-16 Niobium total ionization cross section for the 5 outer shell electrons.......................................................

Fig. 3-17 A typical electron's movement inside the ECR-magnetic field coupled with the longitudinal electric field.

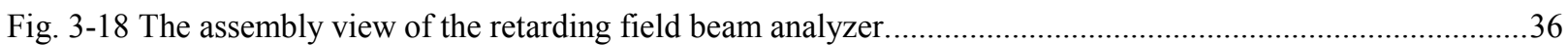

Fig. 3-19 The grid potential for retarding field ion energy analyzer ..........................................................38

Fig. 3-20 The circuit diagram for retarding field ion energy analyzer ........................................................3

Fig. 3-21 The labview panel screen shot shows an I-V curve during beam test...................................................39

Fig. 3-22 The ion beam energy distributions with different ion beam energies. .............................................40

Fig. 4-1 Absorbed RF power saturates as RF input power increases ...............................................................44

Fig. 4-2 Collector current is almost linear to the absorbed RF power ......................................................4

Fig. 4-3 Niobium ion kinetic energy distribution measured at substrate holder..................................................46

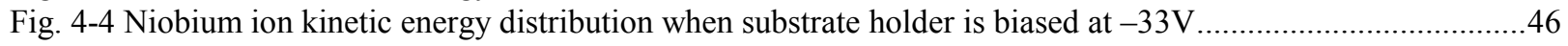

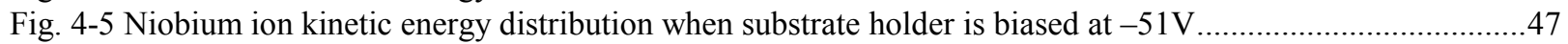

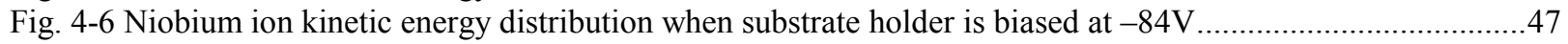

Fig. 4-7 Niobium deposition rate on sapphire versus substrate bias voltage ................................................48

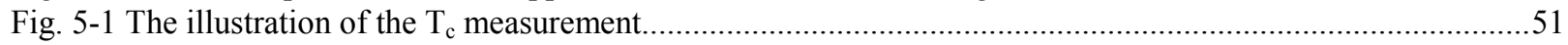

Fig. 5-2 The resistance of a typical copper coil (left chart) ............................................................................51

Fig. 5-3 The $\mathrm{T}_{\mathrm{c}}$ curve measured by a large coil set (upper chart) ................................................................53

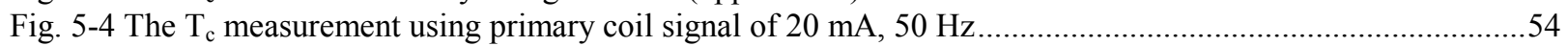

Fig. 5-5 The illustration of the $\mathrm{T}_{\mathrm{c}}$ measurement with phase information .........................................................55

Fig. 5-6 Transition temperature for Niobium thin film on Copper by AC induction measurement ........................55

Fig. 5-7 Niobium thin film RRR measurement by the four point resistive method.............................................57

Fig. 5-8 A typical RRR curve measured on $\mathrm{Nb}$ thin film on sapphire ....................................................5

Fig. 5-9 The making of superconducting coil for upper critical magnetic field measurement ..............................59

Fig. 5-10 The field contour of superconducting coil at center axis .........................................................60

Fig. 5-11 The magnetic field of superconducting coil for center 5 -inches.......................................................61

Fig. 5-12 Niobium thin film cross section on silicon. (SEM) .............................................................62

Fig. 5-13 The GADDS X-ray result shows a well-aligned crystal structure for $\mathrm{Nb}$ thin film on sapphire substrate..62

Fig. 5-14 The AFM picture shows a flat, densely packed, niobium thin film on a sapphire substrate ....................63 


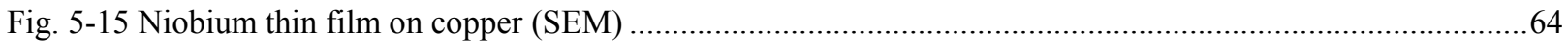

Fig. 6-1 Bias voltage effect on transition temperature measured by inductive method............................................69

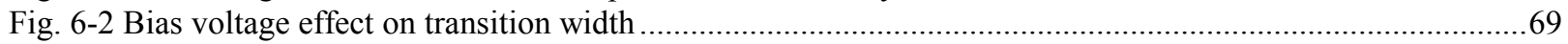

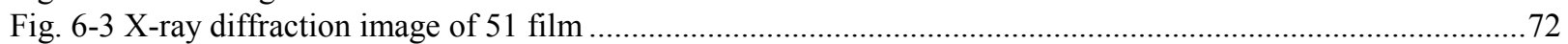

Fig. 6-4 From left to right, and top to down, the AFM images are for $02 \mathrm{~V}, 25 \mathrm{~V}, 51 \mathrm{~V}, 72 \mathrm{~V}, 100 \mathrm{~V}$ films, image sizes

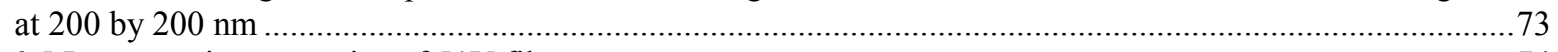

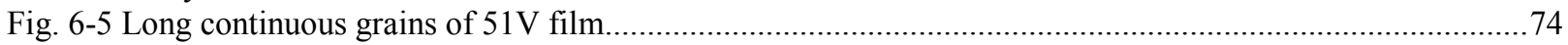

Fig. 6-6 Electropolished Copper surface under optical microscope ……..............................................................75

Fig. 6-7 One emitter at different magnifications (contains $\mathrm{Cu}, \mathrm{Fe}, \mathrm{Ni}, \mathrm{Cr}$ ) ........................................................75 


\section{List of Tables}

Table 3-1 Cut ratio c/d $d_{0}$ vs. S-parameter. (WR284 e-plane cut, 2.45GHz) .......................................................23

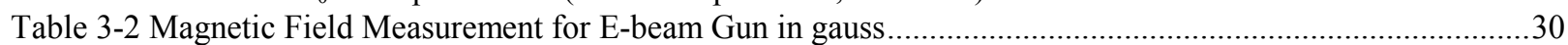

Table 4-1 The absorbed RF power and the current level of second current source .............................................43

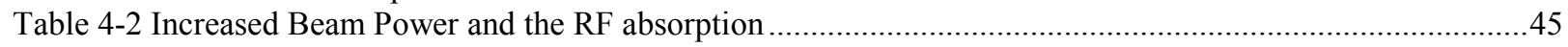

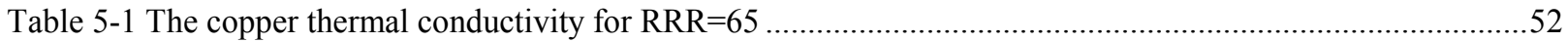

Table 5-2 The calibration data for magnetic coil with $100 \mathrm{~mA}$ current .......................................................60

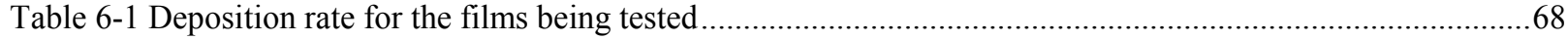

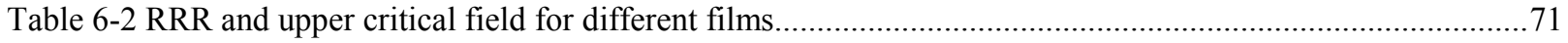

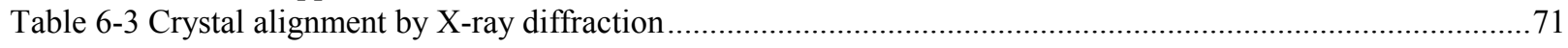




\section{Chapter 1. Thin Film Cavities for Superconducting Radio Frequency Particle Accelerators}

\section{1-1. Introduction}

Currently, DC magnetron sputtering is the working process to make niobium thin film cavities [1]. Due to the high field limitation on copper cavities coated by magnetron sputtering [2], many new thin film techniques are being explored in order to improve the high field limitation. For niobium thin films, there are DC post magnetron processes, biased DC magnetron deposition, and vacuum arc coating. The thin film materials other than niobium that have been investigated are $\mathrm{Nb}_{3} \mathrm{Sn}$, $\mathrm{NbTiN}$ and the recently discovered $\mathrm{MgB}_{2}$. While these different processes or materials all share their advantages and disadvantages as detailed in chapter Two, either the processes are quite limited or the thin film growth is not well understood. For niobium, the thermal energy for evaporated atoms is about $0.1 \mathrm{eV}$, while the sputtering process has an atom energy around $10 \mathrm{eV}$. Energetic condensation for niobium with controllable deposition energy is a totally new approach to investigate the correlation between the niobium thin film growth and its physical properties.

Energetic deposition or condensation is a coating process with the deposition energy well defined in a range above the thermal deposition process and below the ion implantation process. The energy involved in the energetic deposition is variable and depends on the material. Typically, the energy for energetic deposition ranges between 10 and $100 \mathrm{eV}$. In the energy range well above $200 \mathrm{eV}$, niobium atoms tend to induce back sputtering resulting in lower deposition rate, thus offering less opportunity to explore energetic deposition.

In this chapter, I summarize the Superconducting Radio Frequency based accelerators and their physics applications, briefly describe the basics of RF superconductivity and make arguments for niobium thin film technology. Most of the details can be found in ten workshop proceedings dedicated to SRF technology since 1981. 


\section{1-2. Current Implementations in Particle Accelerators}

It is absolutely not an exaggeration to say that radio frequency superconductivity is the fastest growing technology for particle accelerators. Numerous particle accelerators constructed around the world use or have used accelerating structures based on RF superconductivity. Those particle accelerators are used in a wide range of exciting new physics and industrial applications, including elementary particle physics, nuclear physics, synchrotron radiation light sources, and free electron lasers. They are described in 1997 textbook [3]; but since there has been much progress and there are future projects under consideration, it is necessary to provide a summary here to motivate the pursuit of author's thesis project.

RF superconductivity really shines in heavy ion linacs. It acts as the energy booster for tandem accelerators. Because of its low cost, high flexibility and exceptional beam quality, many SRF based heavy ion linacs found homes at universities and national labs. They are SUNY Stony Brook, Kansas State University, Florida State University, University of Washington, Australia National University, and at many other government laboratories around the world such as America's Argonne National Lab, Japan's Jaeri, Italy's INFN, etc.

For medium energy electron accelerators, SRF based linacs recirculate beams for many turns with highest stability and high average beam current in continuous mode. The beam quality from SRF linacs is far-superior to conventional accelerators in terms of beam emittance, beam energy stability and energy spread [3]. Due to these advantages, the Continuous Electron Beam Accelerator Facility (CEBAF) at Jefferson Lab is one of the two premier accelerator facilities for nuclear studies [4].

Also because of the excellent beam quality and the energy flexibility, the Free Electron Laser (FEL), the once big technology contender for the shelved 'Star War', is the natural fruit from SRF based recirculating linacs. America's Jefferson Lab and Japan's Jaeri provide 1-3kW average laser power for various industrial applications $[5,6]$. Building on the still evolving SRF technology, the Jefferson Lab FEL is aiming for $10 \mathrm{~kW}$ or even $100 \mathrm{~kW}$ laser power [7]. A prototype Test Facility for TeV Energy Superconducting Linear Accelerator (TESLA) is one of 
the main projects pushing SRF research and development in recent years. FEL based on TESLA Test Facility (TTF) is under construction to use the now considered much improved SRF technology [8].

FEL is not the only light source that scientists are building using SRF technology. Many synchrotron radiation light sources including Taiwan light source [9], Canadian light source, DIAMOND in England, SOLEIL (350 MHz) in France, have all started to use SRF based accelerator technology.

High-energy physics storage rings have been using SRF extensively and fruitfully. The storage rings at Cornell (CESR upgrade), KEK (TRISTAN to B-factory), CERN (LEP to LHC), and DESY HERA, all benefited from SRF technology in their respective energy regimes.

Due to the increasing excitement about the use of neutrons for material science studies, the Spallation Neutron Source is being constructed at Oak Ridge, Tennessee, USA. The machine uses SRF technology for its medium beta and high beta proton linac. As its planning committee pointed out, the SRF has advantages of excellent beam quality, machine flexibility, and upgrade capability and overall cost reduction [10].

The TESLA mentioned earlier shows the capability of SRF to be overwhelmingly superior. The TESLA's overall efficiency from electric power to beam power reaches $25 \%$ compared to 3 $10 \%$ in normal conducting design [3], which is crucial in modern days as environmental concerns are increasingly important both politically and economically. Another issue for high-energy machines is the peak RF power requirement. To power such high-energy beams, the klystron number can be reduced to about 600 from 2,000 for a conventional machine, a real cost reduction. On the physics side, the advantages include high luminosity with high beam quality, more flexible detector design and very low dark current from the accelerator itself. As the biggest accelerator project since SSC, TESLA is conducting further cost reduction studies [11].

There are a few future SRF accelerators that are in the concept phase [12]. Rare Isotope Accelerator is being explored at Argonne National Lab [13]. For the Muon collider/Nutrino factory [12], a feasibility study of accelerating structures is underway at Cornell [14]. 
The successful running of CERN LEP2 and the future muon collider are two of the greatest examples to show the benefits of the thin film technology used for RF superconductivity. LEP2 uses 288 Niobium coated cavities out of 336 total cavities [15]. Mainly due to $\mathrm{Nb} / \mathrm{Cu}$ 's low cost, and the requirement of lower accelerating frequency, the superconducting cavities for the Muon Collider/Neutrino Factory Project will use a sputtering technique to produce the low frequency resonant cavities [14].

\section{1-3. Key Parameters and Processes}

SRF for accelerator applications include the research areas of materials, science and engineering. From surface resistance to field limitations, from low temperature physics to RF microwave transmission, and from vacuum science to beam dynamics, an integrated effort is required for a successful particle accelerator to be based on RF Superconductivity. In this section, I will focus the discussion on basic SRF in terms of low temperature superconductivity and electromagnetism, which is closely related to what this thesis research is trying to improve. Several textbooks are good sources for general superconductor physics $[16,17,18]$.

\section{Accelerating field}

Most modern accelerators use microwave resonant cavities to accelerate the charged particles. One of the parameters to characterize the accelerating structures is the accelerating gradient, $\mathrm{E}_{\text {acc. }}$. Of course, there is $\mathrm{E}_{\mathrm{pk}}$ and $\mathrm{H}_{\mathrm{pk}}$, which denote the highest electric and magnetic field inside resonant cavity. $E_{\text {acc }}$ reflects the energy gain per unit length for a singly charged particle traveling through the resonant cavity. In an ideal situation, one can keep feeding the power to the resonant cavity until the peak magnetic field reaches the critical RF magnetic field, a little higher than the thermodynamic critical magnetic field for niobium. (A meta-stable superconducting state under superheated critical magnetic field.) For a typical resonant cavity, the theoretical maximum accelerating gradient is about $55 \mathrm{MV} / \mathrm{m}$ [3]. The average $\mathrm{E}_{\text {acc }}$ is about $25-30 \mathrm{MV} / \mathrm{m}$ for working accelerating cavities based on solid niobium material.

\section{Surface Resistance}

While the DC resistance for a superconductor is zero, the RF resistance, more accurately the 
RF surface resistance, will not be zero. The reason it is called surface impedance is that the surface superconducting current doesn't completely stop the external field from getting into the surface layer. There will always be penetration as long as the electron has mass or inertia. Following the two fluid model, it is the 'leaking' electromagnetic field that accelerates the normal conduction electrons back and forth. Thus there is power loss, which can be described as surface resistance.

Using Maxwell equations at the surface boundary, the penetrated electrical field is about:

$$
E \sim \frac{d H}{d t}=\omega H
$$

And, the current is about

$$
j=n_{n} \cdot \sigma \cdot E \sim n_{n} \sigma \omega H
$$

where $\sigma$ is the normal conducting electrical conductivity. The $n_{n}$ denotes the number density of normal conduction electrons, which should be proportional to (Boltzman factor)

$$
e^{-\frac{\Delta}{k_{b} T}}
$$

where $k_{b}$ is the Boltzman constant, $T$ is the temperature and $\Delta$ is the energy gap.

So, the power loss is

$$
\text { Power }_{\text {loss }} \sim \sigma \omega^{2} H^{2} e^{-\frac{\Delta}{k_{b} T}}
$$

Using the form:

$$
\text { Power }_{\text {loss }} \sim \frac{1}{2} R_{s} H^{2}
$$

yields for surface resistance $R_{\mathrm{s}}$ :

$$
R_{s}=A \omega^{2} e^{-\frac{\Delta}{k_{b} T}}
$$

If there are defects, then even if the temperature is close to zero, the $R_{\mathrm{S}}$ doesn't change too much, which results in residual resistance which is unrelated to rf frequency and temperature.

$$
R_{s}=A \omega^{2} e^{-\frac{\Delta}{k_{b} T}}+R_{\text {res }}
$$

The more accurate surface resistance comes from numerical computations based on BCS microscopic theory. It nevertheless is close to the formula above after data fitting. 
Since the material specie determines the BCS surface resistance intrinsically, the rest of the improvements are focused on reducing the residual resistance and other factors like trapped magnetic field induced surface loss.

\section{Thermal RF heating}

While one can make a perfect resonant cavity by extensive effort in the laboratory, there will be defects in real manufacturing, especially microscopic defects. The defects will heat up the local area by high surface current induced by the electromagnetic field. The thermal conductivity of the resonant cavity limits the amount of heat that can be dissipated to the cooling agent. Eventually, before the magnetic field exceeds the critical value, the temperature will exceed the transition temperature $T_{c}$, if one keeps increasing the stored energy. The local area loses the superconducting state, and then it becomes a run away process (quench). (The increased temperature certainly reduces the critical magnetic field, which in turn will cause the quench.) To efficiently cool the resonant cavities, one can try to reduce the wall thickness while maintaining sufficient mechanical strength, improve the thermal conductivity of the cavity material, use thin film technology, or use a different material with higher $\mathrm{T}_{\mathrm{c}}$ and higher critical magnetic fields. I will list these new efforts later in this chapter and in chapter two, in order to identify the significance of the thesis project.

\section{Multipacting}

In RF resonant cavities, stray electrons can hit the cavity surface, creating secondary electrons. The secondary electrons, which in turn, can be accelerated by RF fields, absorb the RF energy and hit the wall surface to create more secondary electrons. When these secondary electrons happen to hit the same area, the absorbed RF energy heats the surface, thus creating a local hot spot. That not only limits the accelerating gradient, but also creates more RF surface loss

eventually leading to a thermal quench. By adjusting the resonant cavity shape, the multipacting can be reduced. It can't be totally eliminated, and whenever it happens, the thermal efficiency surely would help. Also, some resonant cavities such as quarter wave resonators can't change their geometry shape to fully overcome the multipacting. 


\section{Field Emission}

Another important phenomenon limiting the $E_{a c c}$ from reaching its maximum is field emission. When the surface electrical field is strong enough for that small area, typically $20 \mathrm{MV} / \mathrm{m}$, the electrons will start to tunnel out of the metal surface to form a steady current. The field emission current increases rapidly as the field is increased. Like the multipacting phenomenon, it absorbs a great amount of RF power, depositing the heat wherever the electrons hit. Some of the heating is strong enough to cause the resonant cavity to lose the superconducting (SC) state.

\section{1-4. Advantages of Niobium Thin Film Cavity}

Niobium coated Copper cavity has the following features:

\section{Thermal stability}

Bulk niobium has a typical heat conductance of about $75 \mathrm{~W} / \mathrm{m} \cdot \mathrm{K}$ if the purity is high, while the copper is as high as $300-2000 \mathrm{~W} / \mathrm{m} \cdot \mathrm{K}$. For a typical hot spot inside a SC cavity, say, 50-micron size defect, the amount of heat is about $10 \mathrm{~mW}$ if the magnetic field at the defect area is 400 Oe. It is very easy for the copper substrate cavity to conduct the excessive heat to the helium bath. A more accurate simulation using thermal breakdown model for defects inside the resonant cavity [19] sufficiently illustrates the effect of the defect's size and the niobium purity level (represented by RRR, residual resistivity ratio). The following is another simple illustration to showcase the difference between solid niobium and copper.

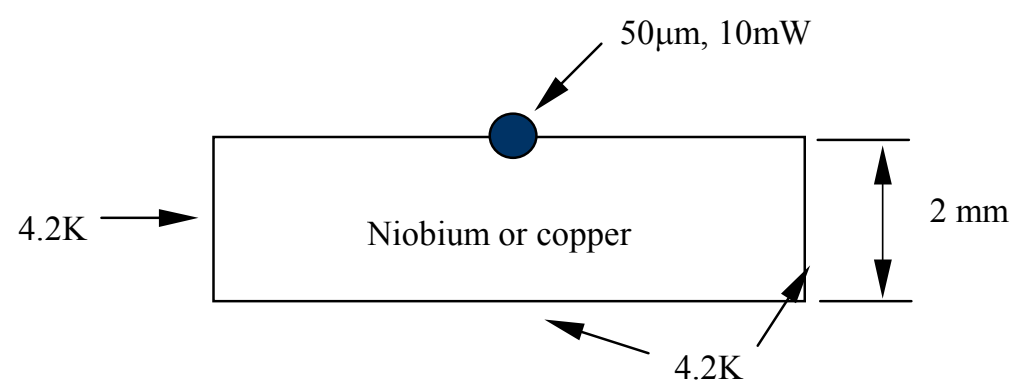

Fig. 1-1 A coarse model for temperature estimation around a $50 \mu \mathrm{m}$ size defect. 
Consider a $50 \mu \mathrm{m}$ defect is buried inside the niobium or copper block, which has the thickness of $2 \mathrm{~mm}$ as we see in a typical niobium resonant cavity. There is 400 Oe rf magnetic field. Assume the block is perfectly cooled in a $4.2 \mathrm{~K}$ helium bath, and the radiation and the RF heating of the top surface are ignored. A rough estimation shows that the temperature gradient is quite large for niobium compared with that for copper.

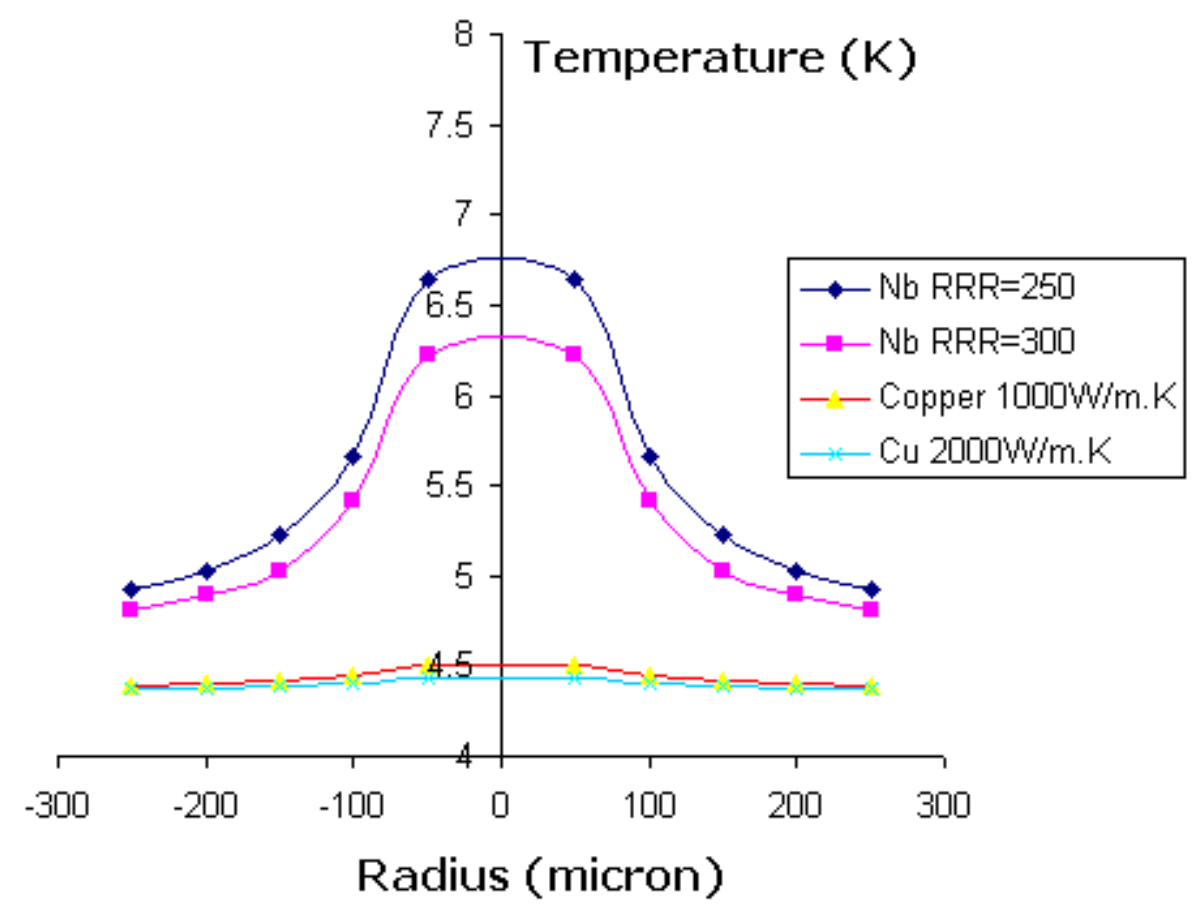

Fig. 1-2 The temperature estimation around a $50 \mu \mathrm{m}$ size defect.

As we have seen in the previous section, multipacting and field emission could cause problems to resonant cavities. The large thermal conductivity of copper helps the thin film cavity to be more resilient in those situations.

\section{Low cost}

The copper is a lot cheaper than niobium, about $1 / 10$ the cost of the niobium. Since the dimension of the RF cavity is inversely proportional to its resonant frequency, a typical $500 \mathrm{MHz}$ cavity needs more than 9 times the material required for a $1,500 \mathrm{MHz}$ cavity with a same cavity thickness. If the thickness is made proportional to diameter, the material per cell would be 27 
times. And the material itself is not the only cost advantage. It is widely believed that the manufacturing cost associated with copper can be substantially lower than that of niobium.

\section{Insensitivity to earth magnetic field trapping}

Residual resistance of the niobium surface is caused by many different sources. For an ideally cleaned cavity, the residual resistance can be caused by trapped magnetic flux, hydrogen dissolved in the surface layer, surface oxides and even some surface roughness. The residual resistance of thin film caused by trapped magnetic field can be up to 10 times less than that of a bulk niobium cavity. (Theoretical calculation shows the loss is inversely proportional to niobium's upper critical magnetic field $\mathrm{H}_{\mathrm{c} 2}$ [3]. Thin film niobium can push $\mathrm{H}_{\mathrm{c} 2}$ as high as 35,000 Oe [1]. While bulk niobium has $\mathrm{H}_{\mathrm{c} 2}$ around 2400 Oe. )

\section{Thin film is potentially free of undissolved inclusions mostly seen in bulk material}

This is mostly a metallurgical issue. During the pressing, rolling and melting, etc., the niobium sheet or the copper sheet will have some kind of micro-inclusions. Typical inclusions can be elements such as steel, nickel, and some compound oxides. They don't necessarily decrease the RRR of the metal sheet, but they show up from time to time, even with vigorous etching. Although they can be controlled well, they do increase the cost for better raw material and also the cost for extra processing during the cavity manufacturing. One simple example is that the inclusions are very easily introduced to the niobium sample from machine tools [20]. Although that kind of inclusions can be etched away, the inclusions caused by precipitation during cooling process can be deep inside the niobium material. That can happen when the amount of some compound dissolved while the $\mathrm{Nb}$ is molten exceeds the solubility during cooling.

Vacuum deposition of thin film is known to produce excellent surface quality except the possible degradation of performance due to the increased BCS surface resistance. 


\section{Chapter 2. The Comparison between the Existing Coating Techniques and the Energetic Condensation}

The coating of Niobium on copper by sputtering has been extensively explored at CERN. More comparisons will be made with that particular technique due to the large amount of data. Some research results of sputtering coating show how the energetic deposition can be implemented to overcome the limitations of sputtering coating.

\section{2-1. Sputtering niobium thin film on copper}

\section{2-1-1. Current status of $\mathrm{NbCu}$ thin film cavities}

The first two kinds of defects in $\mathrm{NbCu}$ cavities that are created in production [21] are the defects at the interface inherently caused during $\mathrm{Cu}$ surface preparation and the foreign particles on the $\mathrm{Nb}$ surface. When a cavity is carefully prepared, i.e. the $\mathrm{NbCu}$ cavity is free from the above two defects (macro defects), it still shows a great Q-value degradation which may be attributed to many loss mechanisms summarized as Non-Quadratic RF losses [22]. A recent experiment showed the copper substrate plays a very important role in resulting cavity performance. When the copper substrate cavity is prepared by the spinning method, the Niobium thin film cavity shows no significant Q-degradation within $15 \mathrm{MV} / \mathrm{m} \mathrm{E}_{\mathrm{acc}}$ [2]. It is fair to say, the Niobium thin film cavity is reaching its goal to replace the bulk Niobium cavity. However, the physics of Niobium thin film still needs to be explored further. Specifically, the Q-degradation mechanisms still remains a question.

\section{2-1-2. Non-Quadratic RF losses}

The Q-degradation actually exists in both bulk niobium and in thin film cavities. As named by the Durand and Weingarton in their pioneer work, the anomalous RF loss is known as NonQuadratic Loss (NQL), which is caused by the increased external RF field, and contributes in addition to the BCS and residual surface resistance [22]. 


$$
\begin{aligned}
& \mathrm{P}=\int \mathrm{R}_{\mathrm{S}}\left(\mathrm{B} / \mu_{\mathrm{O}}\right)^{2} / 2 \cdot \mathrm{ds}+\text { others } \\
& \mathrm{R}_{\mathrm{s}}=\mathrm{R}_{\mathrm{bcs}}+\mathrm{R}_{\mathrm{res}}+\mathrm{R}_{\mathrm{NQL}} \cdot \mathrm{B}
\end{aligned}
$$

The "others" represent the non-RF losses. The surface resistance $\mathrm{R}_{\mathrm{s}}$ represents three kinds of RF losses: BCS, residual and NQL.

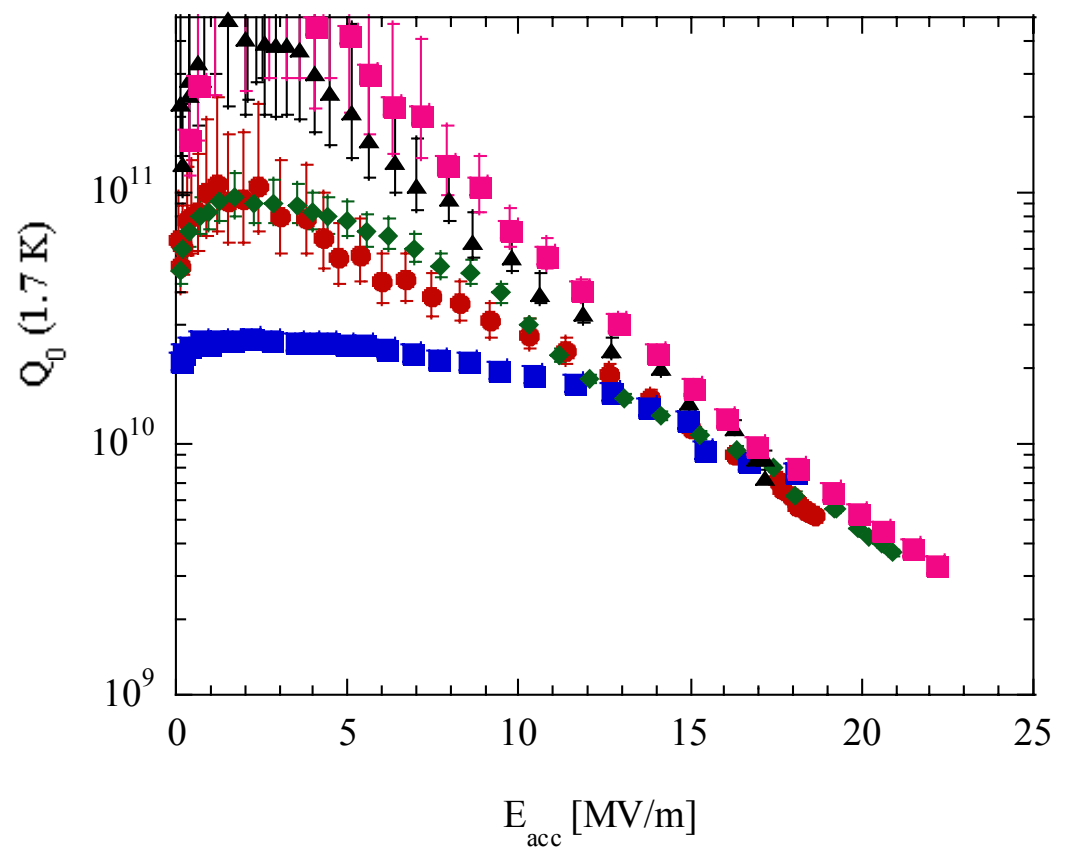

Fig. 2-1 The Q-degradation Seen on Sputtering-coated Nb Copper Cavity (Courtesy of CERN) [2] The different symbols denote different cavities.

\section{2-1-3. Cause of NQL}

There is no clear view so far to fully explain the NQL of Niobium cavities. One possible explanation is hypothesized vortices inside the superconductor [23].

After the two macro defects are removed, the rich density of grain boundary and the intrinsic defects dominate the thin film. This is clearly revealed by TEM analyses at CERN [22].

Malev and Weisser analyzed the data available from CERN's $\mathrm{Nb}$ thin film and revealed that the stimulated desorption mainly contributes to the oxygen and carbon oxide partial pressure to 
$10^{-7}$ mbar during the argon discharge sputtering [24]. However, several analysis methods show no concentrated oxygen migrated to the grain boundary and the interface between the thin film and substrate [22]. Whether there is oxygen or not is still in dispute [25].

There is no strong proof showing whether the intrinsic defects are filled with oxygen or not. The intrinsic defects are believed to be conducting nanometer size junctions, and they decrease the critical magnetic field of the superconducting thin film [22]. It resembles the intragranular weak links in the superconductor. The intrinsic defects are also believed to trap the RF magnetic flux, which contributes significantly to the NQL.

Weingarten introduced hysteretic magnetic flux penetration into the top surface layer to explain the NQL loss [26].

One interesting proposal by Knobloch states that the impurity between grain boundaries and local magnetic field enhancement contributes to the Q-slope after Buffer Chemical Polishing (BCP) process of bulk $\mathrm{Nb}$ cavity, while an electropolishing process greatly improved the Q-slope [27]. The proposed mechanism could help explain the Q-degradation for $\mathrm{Nb} / \mathrm{Cu}$ cavity.

Possible explanations for the abnormal loss in thin film cavities are:

1. The local nc heating up? NO

2. The high density of grain boundary? YES

3. The intrinsic defects? YES

The intragranular weak links? Possible

4. Oxygen? No conclusion.

5. The thermal impedance between copper substrate and $\mathrm{Nb}$ film? No conclusion. Mismatch between those two $\rightarrow$ higher temperature than He bath $\rightarrow$ more BCS loss with the increased RF power.

\section{2-1-4. The coating temperature effect in sputtering}

The substrate temperature is one of the most interesting parameters during the argon discharge sputtering process. There is a focused description of temperature effects on full cavity sputtering 
at CERN, Geneva [28]. An optimum range for the sputtering temperature is at $150^{\circ} \mathrm{C}-175^{\circ} \mathrm{C}$ for better $R_{s}$. There is no other information like the film morphology available for further discussion. In [29], the Orlandi group has reported that sputtering on substrate temperature of $550^{\circ} \mathrm{C}$ leads to a quite large grain size, $0.2 \mu \mathrm{m}$, and a RRR of 35 . From the report, we also know the deposition rate was higher than the lower temperature case [29].

From early morphology studies, the film structure is highly correlated with the deposition rate and the adatom surface mobility $[30,31]$. By increasing the incident atom energy and incident flux, we can expect increased substrate temperature right at the surface, and higher adatom surface mobility, which leads to the larger grain size. Based on this and the findings in [29], Schucan, et., al., have tried to use Ar/He mixture as working gas to increase the adatom surface mobility during niobium deposition [32]. Unfortunately rare gas trapping reduces the grain size and affects the grain growth. The investigation of the rare gas trapping of $\mathrm{Ar}, \mathrm{Ke}$ and $\mathrm{Xe}$ is reported in [33].

Following the discussions from 2-1-1 through 2-1-4, we believe that sputtering film technique has the following drawbacks:

1. The working gas is trapped in the film; it may cause intrinsic defects inside of the grain.

2. The impurities of the working gas are not good for the thin film.

3. The deposition energy is low, which does not help to avoid columnar grains.

The RRR of magnetron sputtered film ranged from 5-10, later improved to 10-20 after using krypton as working gas instead of argon. The transition temperature usually occurs at $9.6 \mathrm{~K}$ while many films had unusually wide transition width.

\section{2-2. The other approaches}

\section{$\underline{\mathrm{Nb}}_{3} \underline{\mathrm{Sn} \text { thin film (Cavity) }}$}

In a project at Wupertal University, the initial results of $\mathrm{Nb}_{3} \mathrm{Sn}$ thin films are quite encouraging. From the report of Kneisel and Lewis, the single cell thin film cavity achieved a 
very low residual surface resistance of $2.2 \mathrm{n} \Omega$ at $2 \mathrm{~K}$ with nearly $10^{11} \mathrm{Q}$ value [34]. People still have concerns that the niobium substrate has a relatively limited RRR value, which could limit the maximum accelerating gradients due to thermal quench [1]. This can be true especially in the multi-cell cavity. Recently, the University of Wuppertal decided to quit the SRF field entirely. $\mathrm{The} \mathrm{Nb}_{3} \mathrm{Sn}$ research is now stopped.

\section{$\underline{\mathrm{NbTiN}}$}

Since the NbTiN has relatively high $\mathrm{T}_{\mathrm{c}}$ of $18 \mathrm{~K}$, sputter coated $\mathrm{NbTiN}$ has been tried at Saclay in small samples [1,35]. Some of the samples have a value of $\Delta R_{s} / \Delta B$ as low as $2 n \Omega / m T$. This implies the NQL loss will be very low if this technique is proved further. The NbTiN coating on copper cavities was carried out at CERN [36]. The result showed that the residual resistance could be very high. The cavities also had serious Q-drop at $4.2 \mathrm{~K}$.

\section{CERN laser annealing}

To increase the accelerating field inside the $\mathrm{NbCu}$ cavity, a laser annealing system has been built at CERN [37]. The idea is to increase the crystal grain size of the niobium film using a 308$\mathrm{nm}$ wavelength laser with energy up to $6 \mathrm{~J} / \mathrm{cm}^{2}$. The small sample results show the grain size is enlarged 4 times from $100 \mathrm{~nm}$. The cavity result is still not available.

\section{Italy INFN DC-post magnetron}

It is quite true that increasing the deposition rate during the sputtering process can reduce the impurities in the growing film. The DC-post magnetron method adopts a simple target Niobium pole together with a tunable magnetic field to achieve a uniform film deposition. The experiment has not obtained a uniform quality film yet, while the increased incident flux of Niobium does not improve the RRR very much either. The sputtered single cell cavity has a large residual resistance resulting in a low quality factor. $\left(2 \times 10^{8}\right.$ at $4.2 \mathrm{~K}$. $)$ The result did show the plasma influenced the film growth greatly [38].

\section{$\underline{\text { Vacuum arc coating }}$}

INFN Roma and Soltan Institute formed a joint coating project to investigate the feasibility of a vacuum arc technique. The problem of micro-droplet remains the major concern of this 
technique [39]. In addition, the depositing energy varies from 10-100 eV. The transition temperature ranged randomly from $8.7 \mathrm{~K}$ to $9.26 \mathrm{~K}$ and the niobium thin film with the best result cannot be reproduced for larger thickness.

\section{$\underline{\mathrm{MgB}}_{2}$ coating}

The newly discovered $\mathrm{MgB}_{2}$ [40] has created interest in making thin films or solid cavities [41]. Some $\mathrm{MgB}_{2}$ thin films had surface resistance of $2 \mathrm{~m} \Omega$ for $19.8 \mathrm{GHz}$ at $4 \mathrm{~K}$ [42]. The evaporation process had difficulty to maintain the $\mathrm{MgB}_{2}$ film quality [42]. It is still too early to predict the usability of the new material for particle accelerators.

\section{Biased magnetron sputtering coating}

SRF group at Peking University has tried to bias the substrate during DC magnetron sputter coating. The process is widely believed to be a neutral atom deposition mixed with ions having a broad range of energies. The ions of the sputtering working gas also come to the substrate. The result shows that the film morphology is improved for films with biased substrates, while the superconducting transition extends down to $6 \mathrm{~K}$ and RRR ranged from 7 to 15 . [43].

\section{2-3. The energetic condensation}

Before describing the energetic condensation process, consider the film structure and the possible deposition method.

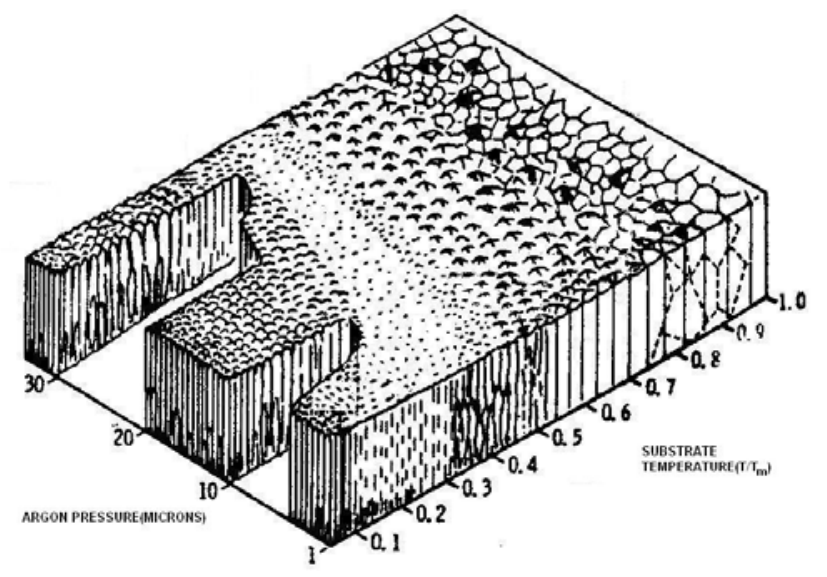

Fig. 2-2 Movchan-Demchishin Zone Model 
Movchan-Demchishin Zone Model shown in Fig. 2-2 is well verified by J.A. Thornton's intensive work [30].

From Thornton's work, we notice that the low argon pressure, high substrate temperature helps to enlarge the column size of thin film. The ion bombardment also assisted the improvement of film structure. All of this leads us to believe if the depositing atom gains more mobility, it will diffuse quickly in the surface, thus is less prone to form the columnar structure.

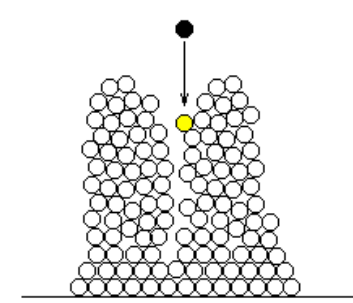

a

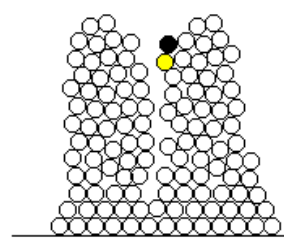

b

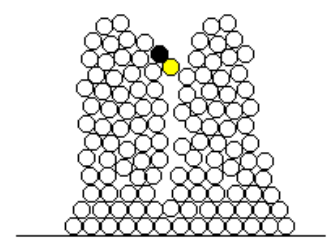

C

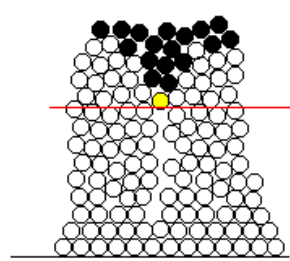

d

Fig. 2-3 The illustration of the surface atom nucleation process to show the columnar forming and atom mobility relation

a), b) the low kinetic energy atom travels and sticks to the surface atoms.

c), d) energetic atom impacts on existing surface, diffuses around.

The following processes can be explored to achieve greater atom surface mobility.

1. High substrate temperature (High $T_{s} / T_{m}, T_{s}$ is the substrate temperature, $T_{m}$ is the melting point of depositing material.)

2. Ion assisted.

3. High-energy deposition.

Metal Vapor plasma deposition

Ionized magnetron sputter deposition

Vacuum arc deposition

Energized metal ion deposition

4. Others: mechanical disturbance, change incident angle, etc.

It is also important to note that any working gas would be trapped in the metal film being formed, thus eliminating working gas such as argon would be a great plus.

Energetic niobium thin film deposition is derived from one of the metal vapor plasma deposition methods and is the most versatile technique among these methods. As noted in the first chapter, it is very unique in terms of controllable deposition energy. There are different ways 
to create energetic niobium flux. The electron cyclotron resonance (ECR) plasma process is believed to be the most suitable process for energetic niobium creation.

The proposed process is similar to the work carried out by Holber [44] and his colleagues from IBM. The basic idea is to use electron cyclotron resonance to create metal ions to do direct film deposition, a process usually called ECR plasma deposition. In Holber's system, copper was the only material studied, and it worked very well. The same process using niobium is the best option for a successful thin film compared with the other techniques.

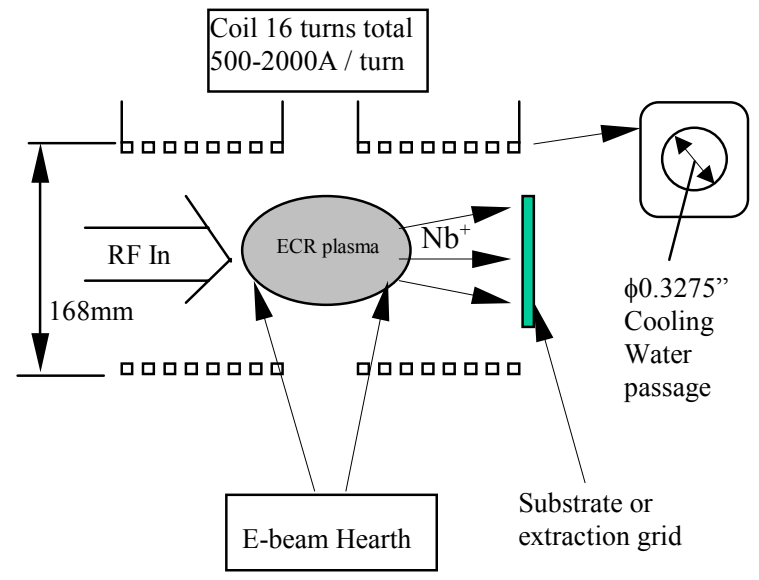

Fig. 2-4 The illustration of e-beam ECR deposition system

The main advantages are:

1. No working gas like argon

2. High vacuum means reduced impurities

3. Controllable deposition energy, and 90-degree deposition flux (Possible to help control the crystal structure)

4. Excellent bonding

5. No macro particles

Electron-Cyclotron-Resonance (ECR) would produce the niobium ions inside a waveguide resonator. Neutral Niobium flux is provided by E-beam evaporation. The system mechanism is illustrated in fig. 2-4 and will be described in more detail in the next chapter. 


\section{Chapter 3. The E-beam ECR Deposition System}

\section{3-1. Overview}

An E-beam ECR vacuum deposition system is developed to study the energetic deposition in vacuum. As shown in figure 3-1, the overall setup consists of several component systems, includes the vacuum system, evaporation system, RF system, and magnetic coil system. The system also incorporates a water-cooling structure. Three 40-psig room temperature water lines are used to cool the magnetic coil, RF magnetron with microwave circulator and the compressor unit for the cryopump. A 80-psig chilled water line is used to cool the E-beam gun hearth.

A thermionic type E-beam gun is used to create niobium evaporation. The E-beam gun has a power level up to $10 \mathrm{~kW}$; and the evaporation rate for niobium can be as high as $150 \AA / \mathrm{s}$ at 12 inch distance.

\section{3-2. Vacuum chamber}

The whole ECR plasma reactor is inside a vacuum chamber as illustrated in figure 3-1. The vacuum chamber is about 21 inches tall with a diameter of 25 inches. Chamber top is supported by a hoist. It is sealed using a Viton O-ring. The chamber is evacuated through two stages. First stage pumping reaches 400 mili-torr using a rotary roughing pump. The roughing line is filtered by a reusable Foreline-trap. The second stage pumping reaches UHV level using a cryopump that has 9,000 liter per second pumping speed. A typical mid $10^{-7}$ torr is achieved after twentyfour hour pumping. In about four weeks, the chamber can reach the base pressure at $8 \times 10^{-9}$ Torr. Water vapor and hydrogen dominate the residual gas content.

The vacuum is monitored using the GP Convectron Gauge, ion gauge and standard Residual Gas Analyzer (RGA). Two glass view ports were installed to allow observation of the E-beam gun. Filtered nitrogen line is connected to the chamber to facilitate chamber purge. During sample exchange, all efforts are taken to minimize the chamber opening time, thus reducing the water vapor absorption on chamber surfaces. 


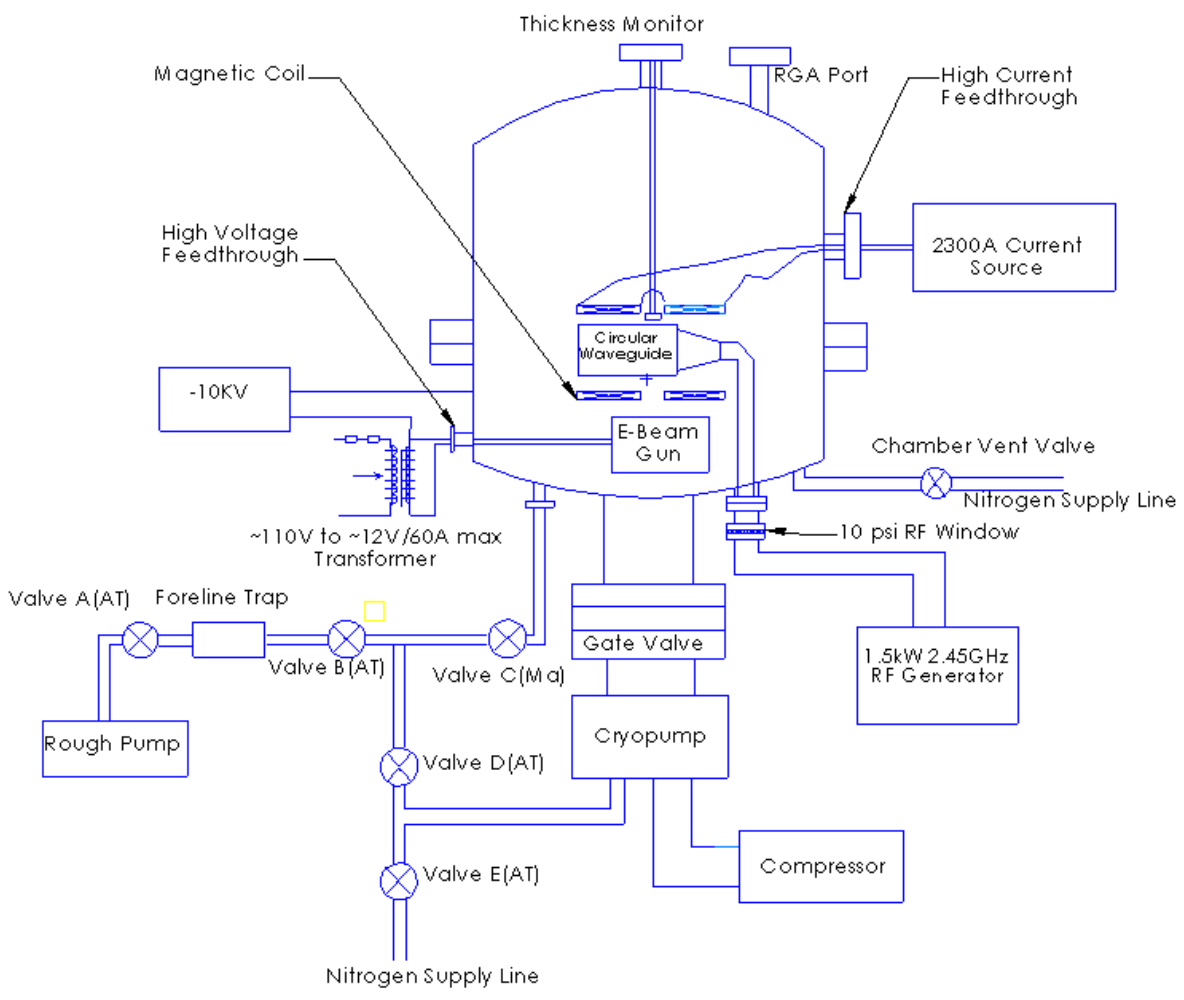

Fig. 3-1 Illustration of E-beam-ECR vacuum system

\section{3-3. The principle of electron cyclotron resonance}

Electrons placed in the external magnetic field will cycle around the magnetic field line if they have velocity component perpendicular to the magnetic field. The electron cycle frequency $\omega$ in external field B is:

$$
\omega=\frac{e B}{m},
$$

where e and $\mathrm{m}$ represent the electron's charge and mass, respectively. If there is periodic electrical field perpendicular to the external magnetic field and at the frequency equal to the electron cycle frequency $\omega$, then the electrons will be constantly accelerated like the electron moving clockwise in figure 3-2. Its path eventually forms an open helix like that in figure 3-2 and $3-3$, and electrons quickly gain energy. 

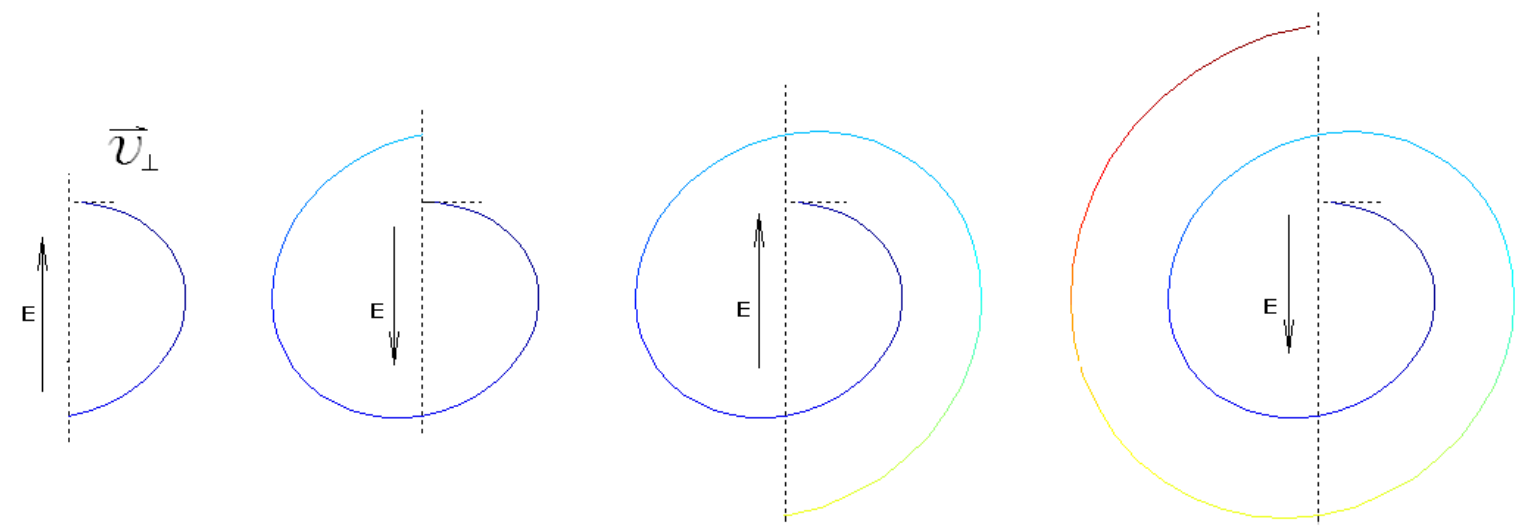

Fig. 3-2 Electron being accelerated clock wisely by periodic electric field
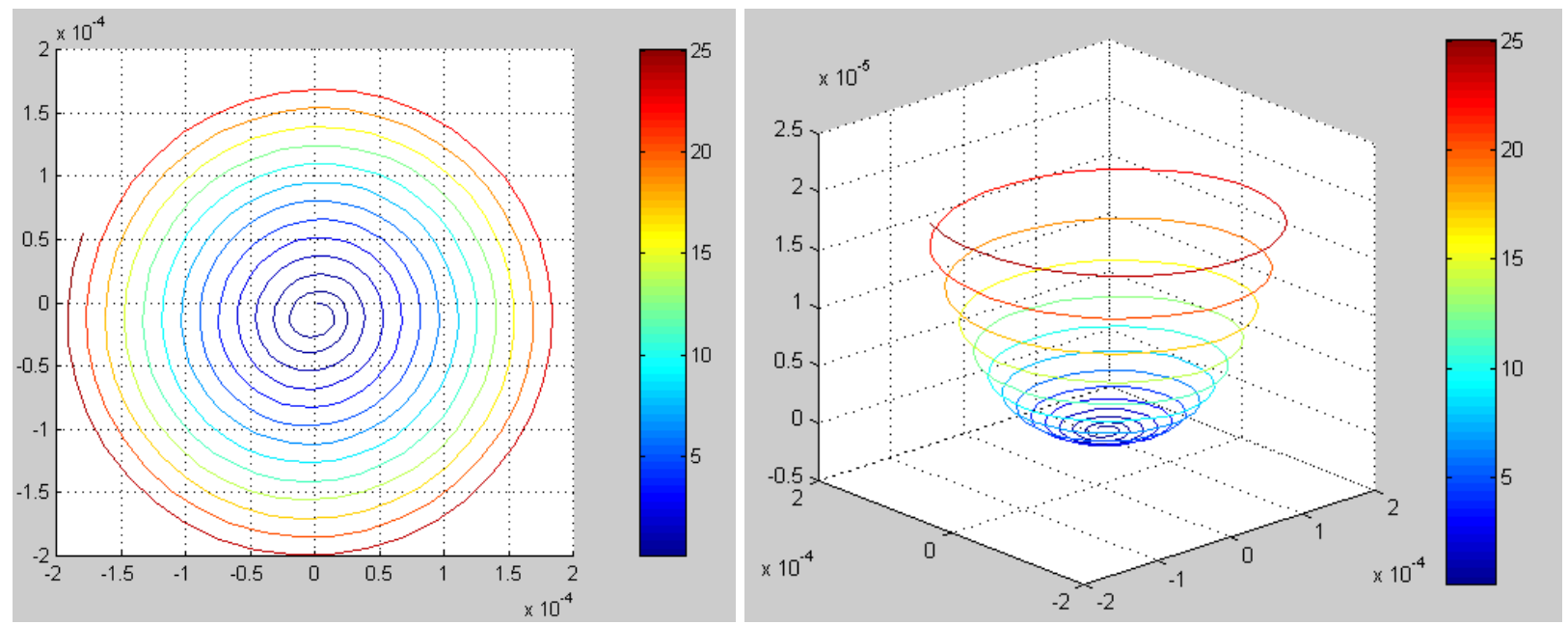

Fig. 3-3 Electron's spiral path in external magnetic field superimposed with perpendicular periodic electric field

When the electrons are accelerated to a large enough energy, they will ionize nearby neutral atoms to create plasma. In practical applications, it is not required to have a uniform magnetic field to create ECR plasma. Most magnetic fields actually have only a limited shell-like ECR region. The RF power absorption mostly happens around the ECR region and keeps the plasma stabilized. Certainly, the more uniform the magnetic field, the greater is the efficiency to make ECR plasma.

\section{3-4. The RF system design}

Since $2.45 \mathrm{GHz}$ is the frequency of consumer product microwave ovens, general availability and low cost are the major reasons to choose this frequency. The RF generator is capable of 
providing $1.5 \mathrm{~kW}$ total microwave power. Figure 3-4 is a diagram showing the simple circuit of this ECR-RF system.

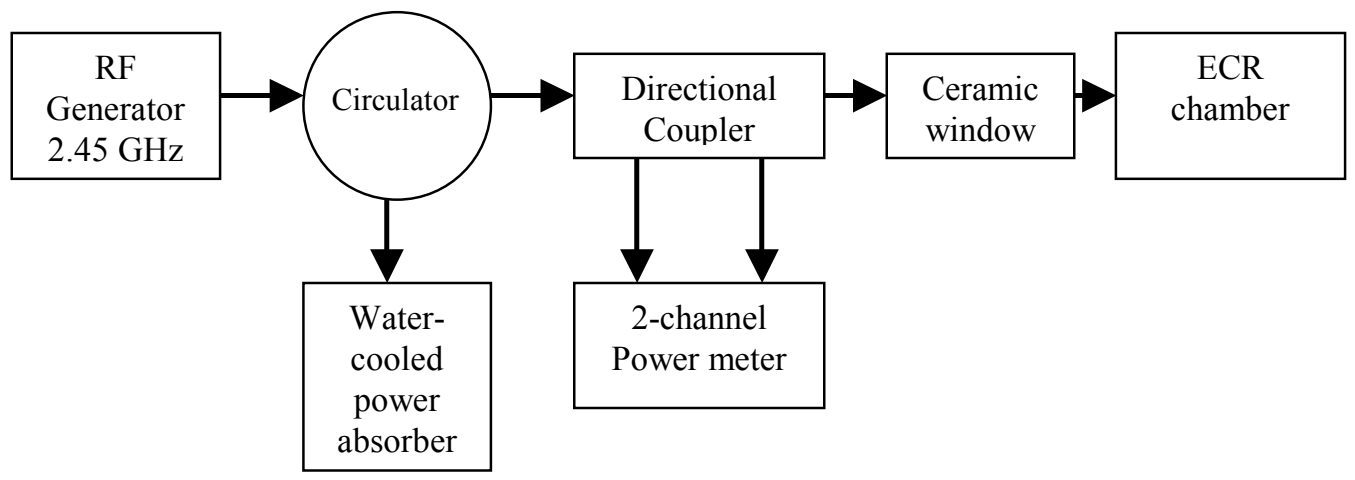

Fig. 3-4 ECR-RF diagram

A simple window is installed to transfer $1.5 \mathrm{~kW}$ RF power to the ECR chamber. The window is designed so that it can be easily replaced as shown in figure 3-5. Two symmetric fins match the transmission line capacitance created by the ceramic block. The ceramic sheet is high purity $\mathrm{Al}_{2} \mathrm{O}_{3}$ with low loss factor of Loss-tangent of 0.0002 and permittivity of 9.2. Two indium wires are pressed at the edge of the ceramic. When the seal is bolt tightened, the indium wire squeezes around to provide both vacuum and RF seal. The window position is not optimized to be the standing wave zero position. Since the vacuum chamber was not designed with RF consideration beforehand, there are limited choices to move the window position. Nevertheless, the window was not a problem within the $1.5 \mathrm{~kW}$ power range, except for one time when it was 'contaminated' by copper scratches left during system modification. The overheating cracked the ceramic, creating a vacuum accident.

Inside the bell jar, there is a 90-degree waveguide bend before the ECR-chamber. The bend is in the E-plane with a 45-degree corner cut as shown in figure 3-6.

The term voltage standing wave ratio (VSWR) is defined as the ratio of the maximum and minimum voltage on a microwave transmission line. A VSWR of unit one means no power 
reflection from the microwave component in a transmission line. S-parameters represent the reflection and transmission coefficients for a multi-port microwave component.

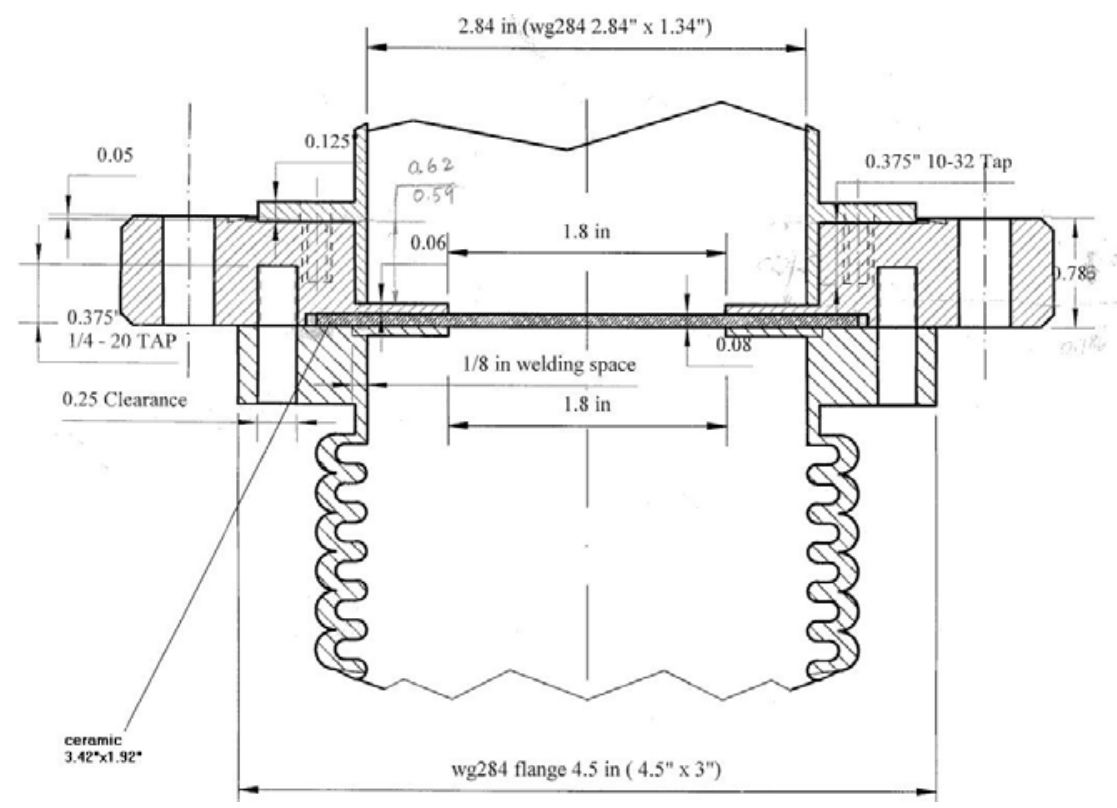

Fig. 3-5 Ceramic window installation

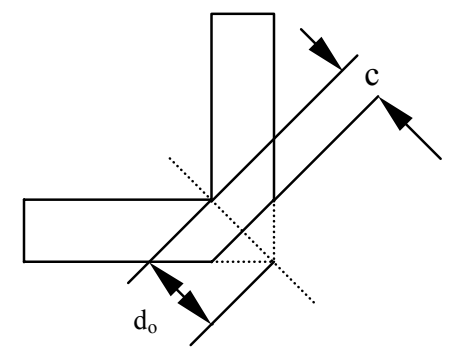

Fig. 3-6 90-degree waveguide elbow 
VSWR can be obtained through formula:

$$
V S W R=\frac{1+S_{11}}{1-S_{11}}
$$

Simulation shows $\mathrm{c} / \mathrm{d}_{0}=0.59$ is the cut ratio for $\mathrm{VSWR}=1$ as shown in table $3-1$.

Table 3-1 Cut ratio c/d $\mathbf{d}_{0}$ vs. S-parameter. (WR284 e-plane cut, 2.45GHz)

\begin{tabular}{|c|c|c|c|}
\hline $\mathrm{c} / \mathrm{d}_{0}$ & $\mathrm{~S}_{11}$ & $\mathrm{~S}_{12}$ & $\Delta \mathrm{S}^{*}$ \\
\hline 0.57 & 0.0258 & 0.9997 & 0.00045 \\
\hline 0.58 & 0.0150 & 0.9999 & 0.00076 \\
\hline 0.59 & 0.0024 & 1.0000 & 0.00070 \\
\hline 0.60 & 0.0121 & 0.9999 & 0.00053 \\
\hline \multirow{2yyy}{*}{0.61} & 0.0247 & 0.9997 & 0.00035 \\
\cline { 2 - 5 } th is & \multicolumn{3}{|c|}{ computation error for S parameters. }
\end{tabular}

The ECR plasma reactor is the heart of the deposition system. One has to note that the basic condition to have a successful ECR is to have the external magnetic field perpendicular to the RF field. External magnetic field for this system is parallel to the RF propagation direction. The RF mode would have to be TE modes inside the plasma reactor. For rectangular waveguide, the mode is $\mathrm{TE}_{01}$. When the simple rectangular waveguide WR284 with the grid opening is used as the ECR coupling resonator, the ionization rate is not high enough to make the plasma selfsustaining. After about six months of trying, it was decided to use a larger circular waveguide as $\mathrm{RF}$ resonator and to open a bigger hole in the circular waveguide to increase the neutral niobium flux. The $\mathrm{TE}_{11} \mathrm{RF}$ mode of a circular waveguide has the electric field perpendicular to the waveguide axis, similar to the $\mathrm{TE}_{01}$ mode in rectangular waveguide.

The switch from rectangular to circular waveguide raises two immediate concerns. The first concern is the taper transition from WR284 to circular waveguide. The second concern is the RF radiation from the big open hole of the circular waveguide. Usually the taper transition isn't very 
difficult if there is enough space. Incorporating the transition inside the chamber requires a very compact design. Considering the manufacturing time and cost, a decision was made to trade off transition efficiency for design simplification and space for the transition.

The taper is showing in figure 3-7. It is to couple the $\mathrm{TE}_{10}$ mode in rectangular waveguide to the $\mathrm{TE}_{11}$ circular mode. The HFSS (Unless otherwise noted, all RF simulations are done with the ANSOFT HFSS program [45].) simulation assumes a two-port structure, port one for WR284 and port two for a circular waveguide. The result shows the $S_{11}$ is 0.1602 and $S_{12}$ is 0.98708 . The field pattern is similar to the one in Fig. 3-8. The $\mathrm{S}_{12}$ of 0.98708 equates to an insertion loss of taper transition of $0.011 \mathrm{~dB}$, which is acceptable.

In radiation simulation, the structure changes to one WR284 input port and one radiation port at the bottom of circular waveguide.

With the open hole on the circular waveguide, an $\mathrm{S}_{11}$ of 0.919 indicates that about $16 \%$ of RF power is radiated out through the open hole.
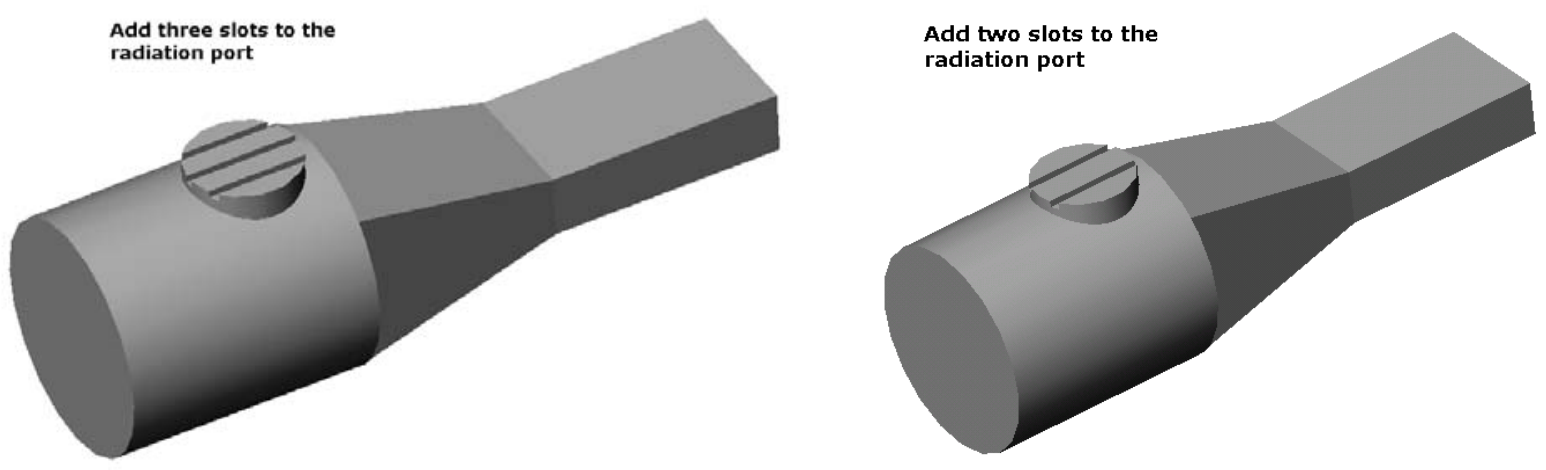

Fig. 3-7 Simulation models with two or three slots to reduce the RF radiation.

If we add three metal slots to the open hole, the radiation simulation shows that the radiation is significantly reduced to only $0.26 \%$. (For $\mathrm{S}_{11}=0.99869$ )

Two slot radiation ports are also modeled indicating that the radiation is as small as $1.9 \%$ (For $\mathrm{S}_{11}=0.99065$ ). The final design uses two slots, which are 0.15 " deep, 0.20 " wide and 1.3 " apart, 
as in figure 3-7.
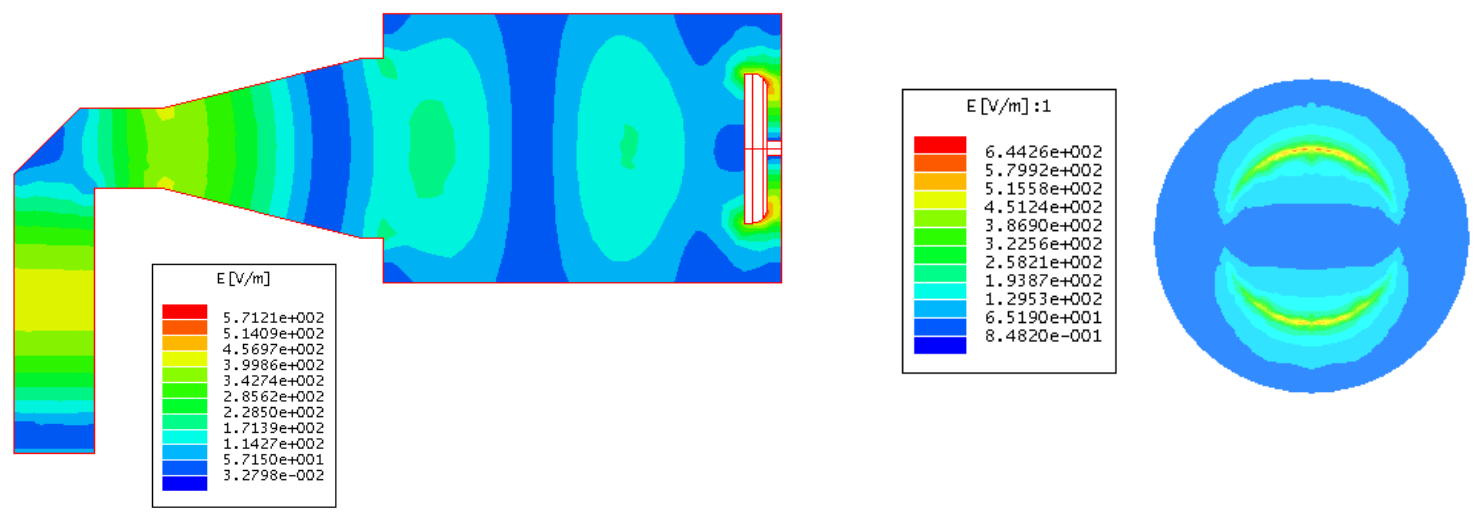

Fig. 3-8 The electric field distribution inside the circular waveguide

Left figure is the field distribution along the center plane; the right figure is the substrate holder surface plane. The field is for the standing wave. The input power is one watt.
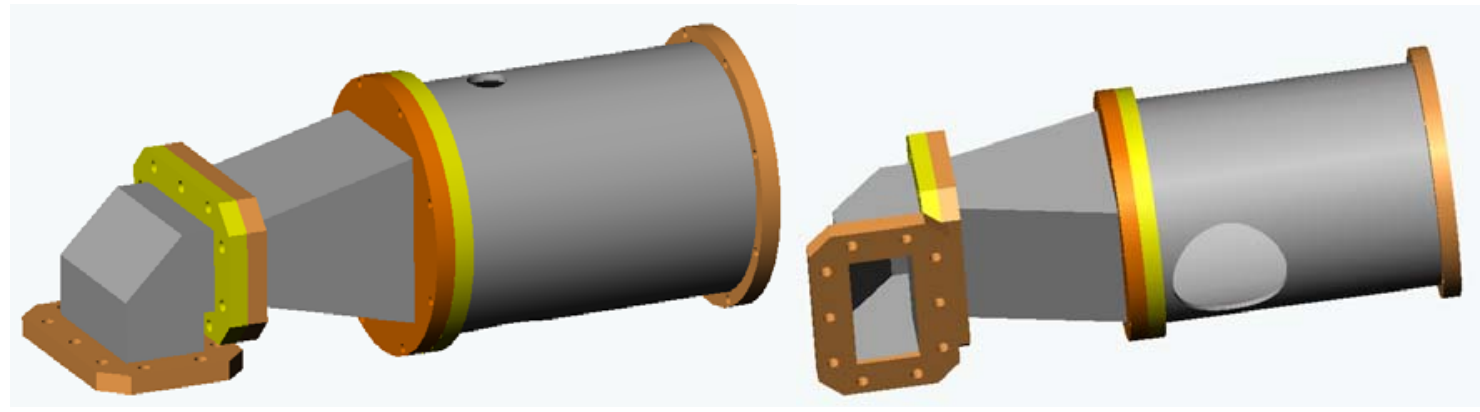

Fig. 3-9 The assembly views of ECR plasma chamber, the slots are not shown in the bottom open hole.

The taper transition and the substrate holder are all integral parts of the whole plasma generator. The size of circular waveguide is carefully adjusted so that the large area of RF electrical field is right in the path of the neutral niobium flux. The substrate holder is out of the line of sight of niobium flux, so that the substrate can only collect ionized niobium. The field pattern on the substrate surface allows some mechanical assembly without disturbing the field too much, as it is shown in figure 3-8. 
Figure 3-9 are two views of the final assembly for the circular waveguide RF system. The top hole is for thickness monitoring of the neutral niobium flux rate.

\section{3-5. The magnetic coil system}

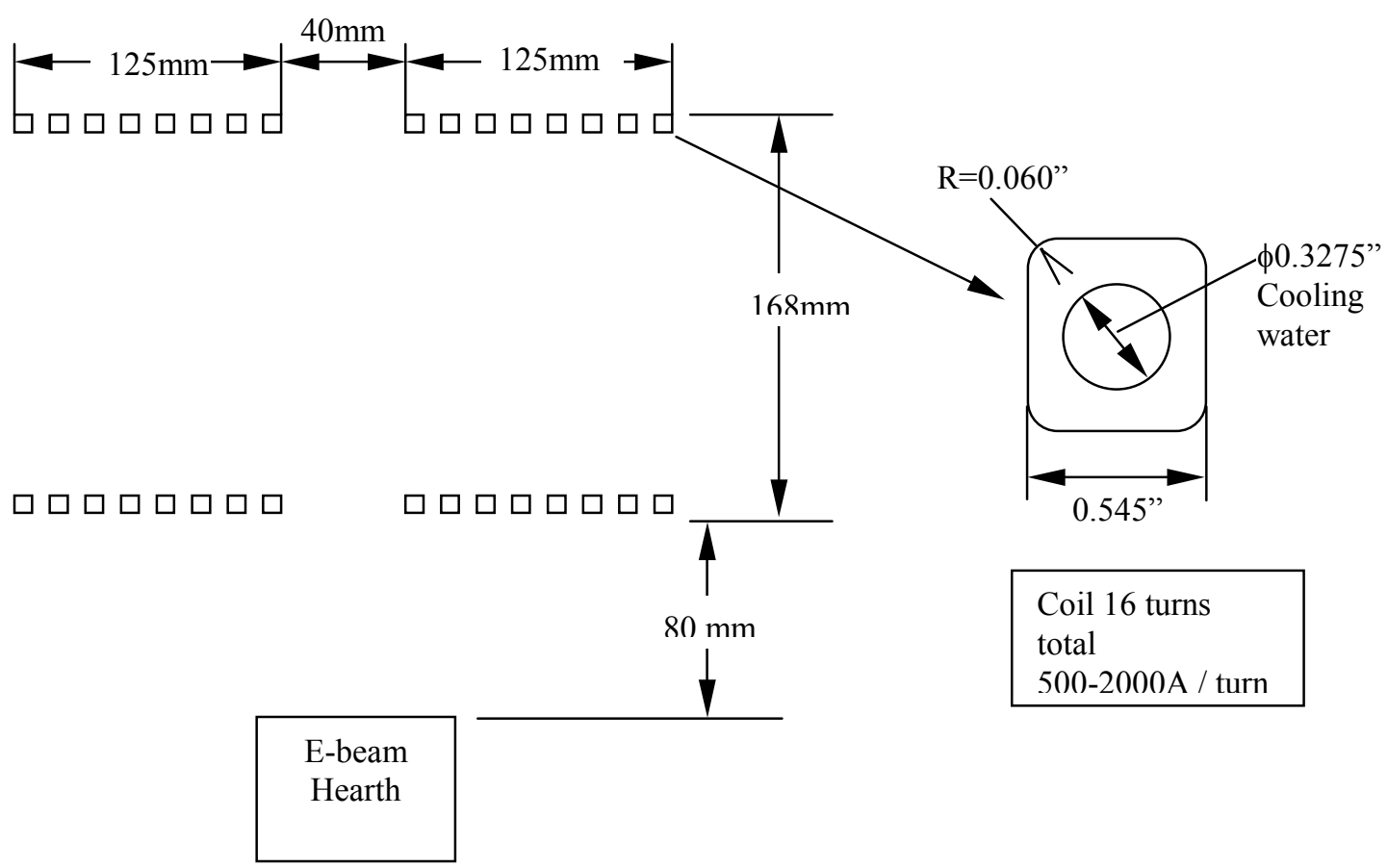

Fig. 3-10 Magnetic Coil versus E-beam gun configuration

The magnetic coils shown in fig. 3-10 achieve the magnetic field required for ECR conditions at 875 gauss. The cooling water is estimated to be adequate for coil power consumption. The cooling water is around 30-psi differential pressure in 3/8-inch tubing.

\section{The magnetic field}

The Poisson/Superfish [46] code is used to design the coil magnetic field. The ideal ECR field configuration is the one designed at Oak Ridge National Lab [47], which maximizes the uniform ECR field and creates axial and longitudinal magnetic field confinement for electrons. It consists of at least three separate coils set to adjust the magnetic field. Unfortunately, the complexity and 
the size of the coil system make it difficult for the UHV deposition chamber. Nevertheless, it provides some ideas for any future improvement of the magnetic field configuration.

Since most of the cheap permanent magnets are not UHV compatible and need a spacious yoke, then they also need strong mechanical support structure. The other disadvantage is that permanent magnets may lose their magnetic strength over time, especially under high heat and irradiation by the electron beam. A high current, water tube cooled electromagnet is the choice for the apparatus.

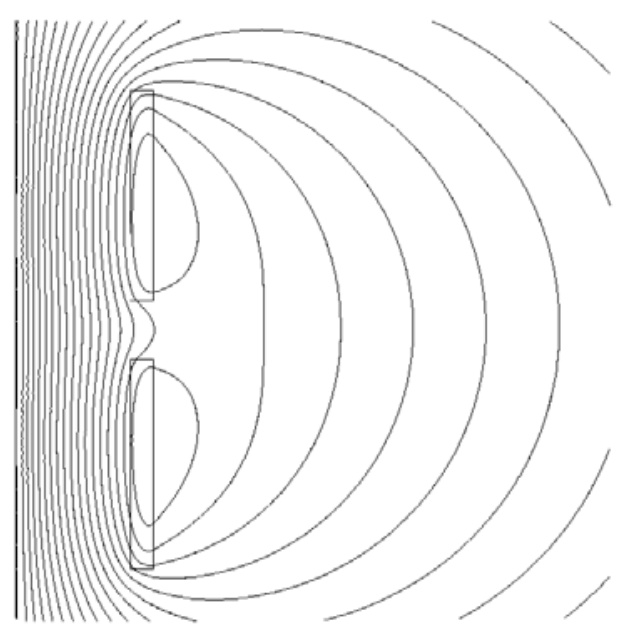

Fig. 3-11 Magnetic Coil field contour simulated by Pandira code, half of the coil cross section is shown.

Figure 3-11 shows the magnetic field contours by the coils. The magnetic field is computed by Pandira code from Poisson/Superfish codes maintained by Los Almos National Laboratory [46].

Figure 3-12 shows the magnetic field value along the different lines parallel to the axis. It is verified by a Hall probe magnetometer.

A coil current of 1,500 ampere through a 0.545 -inch copper tubing as shown in figure 3-10 is selected. The current is provided by two Sorensen power supplies connected in parallel. The maximum current available is 2,300 amperes, hence a current of 1,500 amperes is comfortably in a range to avoid overheating. 


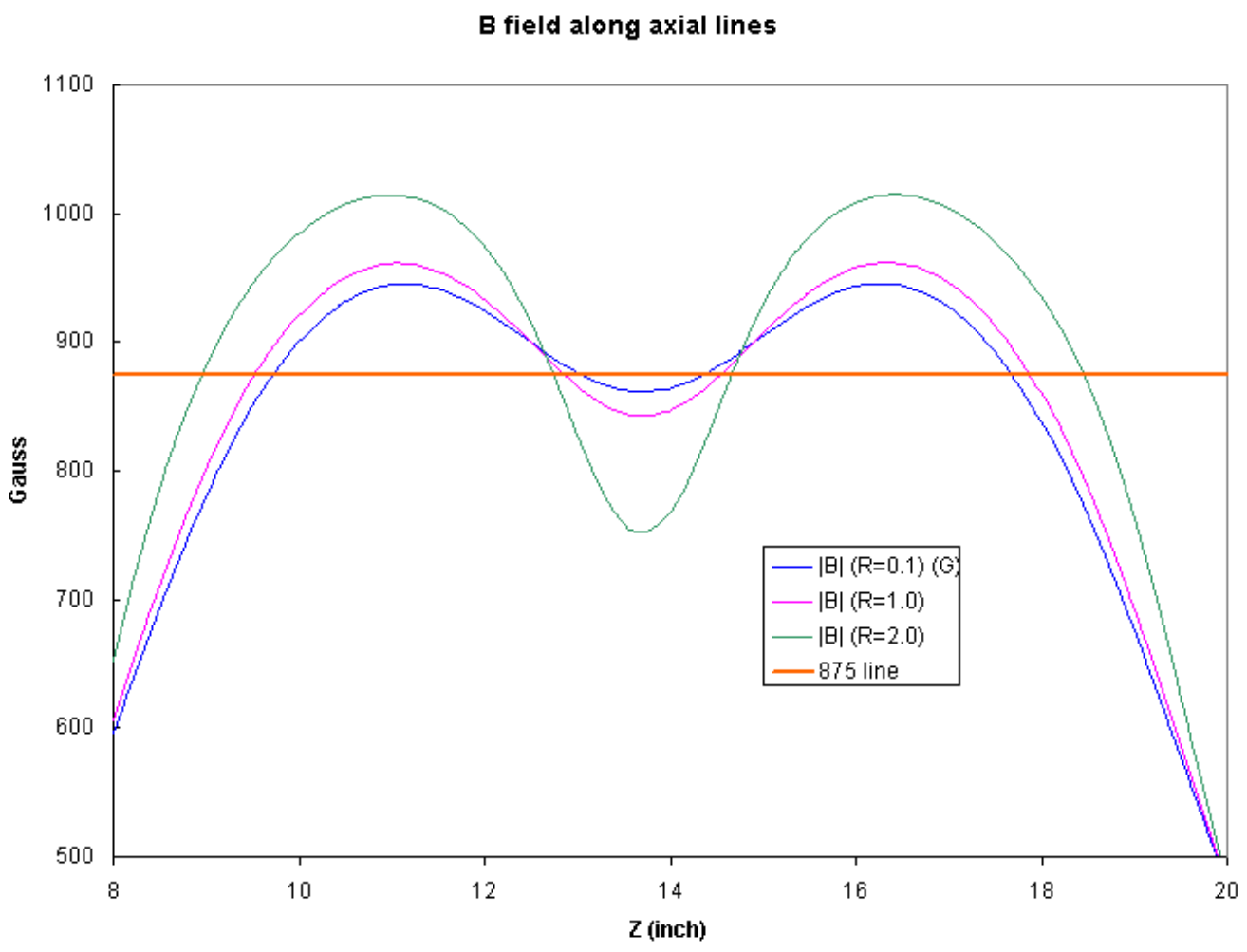

Fig. 3-12 Magnetic Coil field

\section{Coil cooling}

According to the dimensions shown in the figure 3-10. The cross section area is

$$
0.545^{2}-\pi\left(\frac{0.3275}{2}\right)^{2}-\left[(2 \times 0.06)^{2}\right]+\pi \cdot 0.06^{2}=0.210-i n c h^{2}=1.3529-\mathrm{cm}^{2}
$$

There are 17 turns of copper tubing. The inner diameter of coil is 5.5". So the total length of the tubing is 320 inch, which is $814 \mathrm{~cm}$.

The resistivity for copper is $1.913 \times 10^{-6} \Omega \cdot \mathrm{cm}$. The resistance of the coil is $\mathrm{R}=0.0012 \Omega$, and for a 1500 ampere current, there is about a 2 volt drop. The measured number for the coil voltage drop is 0.75 volts for 500 amperes. 
For every centimeter of tubing, the resistance is $1.47 \times 10^{-6} \Omega$, which generates 3.3 watts of heat for a 1,500 amperes current. It has inner surface area of $1.0 \mathrm{~cm} \times \pi \times 0.3275 " \times 2.54 \mathrm{~cm} / "=0.832 \mathrm{~cm}^{2}$. The turbulent water takes away $1.0 \mathrm{~W} /\left(\mathrm{cm}^{2} \cdot{ }^{\circ} \mathrm{C}\right)$.

If the water flow has a velocity of $v(\mathrm{~cm} / \mathrm{s})$, for a $d l$ length of water in tubing, we have total heat exchange after it travels through the whole tubing:

$$
d l \cdot(\pi \cdot D i) \cdot \frac{3.3}{0.832} \cdot \frac{814}{v}=\Delta T \cdot 4.2 \frac{\mathrm{J}}{\mathrm{gram} \cdot{ }^{\circ} \mathrm{C}} \cdot d l \cdot \pi \cdot\left(\frac{\mathrm{Di}}{2}\right)^{2} \cdot 1.0 \mathrm{gram} / \mathrm{cm}^{3}
$$

where $D i$ is inner diameter of tubing, $0.3275^{\prime}$.

So the water temperature rise is

$$
\Delta T=\frac{3696.4}{v}
$$

Water flow inside quarter inch tubing from 50 psig to air has a flow rate of $55 \mathrm{ml} / \mathrm{s}$, which translates to a water velocity of $150 \mathrm{~cm} / \mathrm{s}$ for a $25 \mathrm{psig}$ differential pressure water flow.

This translates into a $25^{\circ} \mathrm{C}$ increase of the water temperature.

\section{Water-cooling of feedthrough}

For a 1/4-inch copper tubing, the cross section is about $0.14 \mathrm{~cm}^{2}$.

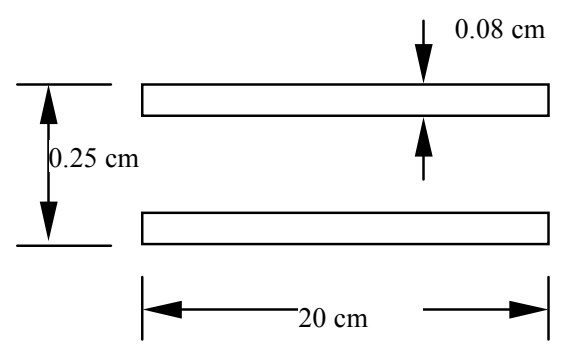

Fig. 3-13 Feedthrough tubing dimensions

The inside surface area for $20 \mathrm{~cm}$ long tubing is $30 \mathrm{~cm}^{2}$. The total resistance is 


$$
R=\frac{1.55 \cdot 10^{-6} \Omega \cdot \mathrm{cm} \cdot 20.0 \mathrm{~cm}}{0.14 \mathrm{~cm}^{2}}=0.222 \cdot 10^{-3} \Omega
$$

A 1,500 Ampere current generates about 500 Watts of heat. Unit area heating is

$$
\frac{500 \mathrm{~W}}{30 \mathrm{~cm}^{2}}=17 \frac{\mathrm{W}}{\mathrm{cm}^{2}}
$$

We know the turbulent water brings away $1 \mathrm{~W} / \mathrm{cm}^{2} \cdot{ }^{\circ} \mathrm{C}$

Similar to the analysis in coil cooling, we get

$$
\Delta T=\frac{681.7}{v}, \text { where } v \text { is the water velocity }(\mathrm{cm} / \mathrm{s})
$$

For water velocity of $150 \mathrm{~cm} / \mathrm{s}$, there is a $4.55^{\circ} \mathrm{C}$ temperature increase for each feedthrough tube.

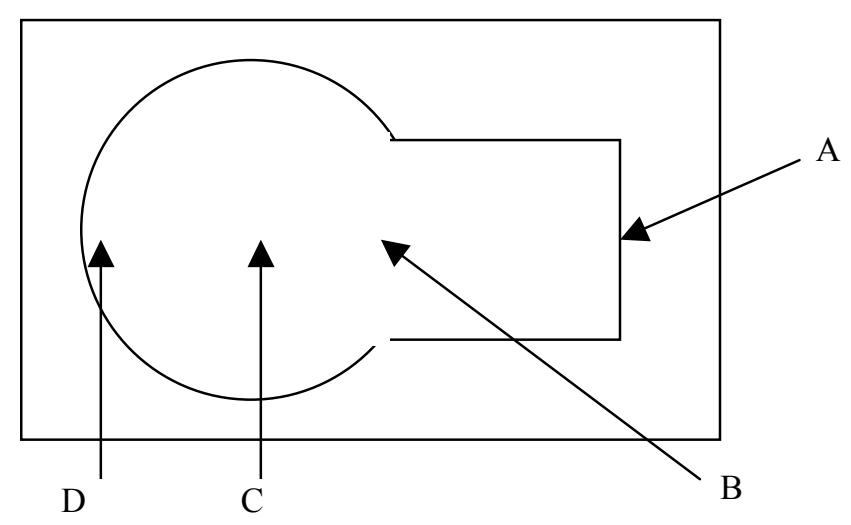

Fig. 3-14 The schematic drawing for E-beam gun test points

Table 3-2 Magnetic Field Measurement for E-beam Gun in gauss

\begin{tabular}{|c|c|c|c|c|}
\hline Test series & $\mathrm{D}$ & $\mathrm{C}$ & $\mathrm{B}$ & $\mathrm{A}$ \\
\hline 1 & 193.2 & 161.6 & 111.0 & 58.0 \\
\hline 2 & 186.8 & 160.2 & 108.4 & 57.2 \\
\hline 3 & 177.6 & 162.0 & 106.7 & 48.7 \\
\hline
\end{tabular}

1: The field from E-beam Gun

2. Iron bars added to the coil

3. Iron bars added to the coil, plus 1,500 ampere current

A, B, C, D denotes measuring points as shown in figure 3-14. 


\section{Stray field interference for E-beam gun}

For a typical operating current (1,500 ampere), the stray field of coil can be as high as 100 gauss at the E-beam gun position, which creates quite a big interference for the E-beam gun. The E-beam gun self-magnetic field is about 200 gauss. After many tries, it was determined that a yoke-like iron bar distributed outside of coils can significantly reduce the stray magnetic field seen by the underneath E-beam hearth. The eight iron bars are about $1 / 2 \times 2 " \times 12 "$ size. They are nickel-plated, and heat-treated in a vacuum furnace. The main reason of plating and heat treatment is to reduce outgassing of the iron bars inside the vacuum chamber. Table 3-2 displays the results of the measurement of the magnetic field at various locations for the E-beam gun.

\section{3-6. The simulation of electron movement}

A small electron tracking code is written to understand the electron's movement inside the electromagnetic field. In addition to the electron's movement, the code also checks the electron's lifetime within the mean free path, and the change of its kinetic energy. The RF field computation is very straightforward. The theoretical result for an ideal circular waveguide with the traveling wave is used. The assumption for the traveling wave is based on full RF power absorption of the plasma operation. The code uses the fourth runge-kutta algorithm [48] to solve the electron's partial differential equation using $\mathrm{C}$ language. The actual $\mathrm{C}$ code and the electromagnetic field computations are shown in appendix B.

\section{The collision between electron and neutral atoms}

First, we estimate the equivalent pressure and the niobium atom density according to the rate of niobium vapor flux.

Considering a small area $\Delta S$ where the deposition rate $\mathrm{R}$ is recorded during time $d t$, there is $d h$ thickness increase.

We have

$$
R=\frac{d h}{d t}=\frac{\Delta S \cdot \Delta h}{\Delta S \cdot d t}=\frac{\Delta V}{\Delta S \cdot d t}=\frac{\Delta M}{\rho_{N b} \cdot \Delta S \cdot d t}=\frac{\Delta N}{\Delta S \cdot d t} \cdot \frac{m}{\rho_{N b}}
$$


$m$ is the $\mathrm{Nb}$ atom's mass, $\rho_{\mathrm{Nb}}$ is the $\mathrm{Nb}$ mass density, $\Delta N$ is the number of $\mathrm{Nb}$ atoms.

$\Delta N=v \cdot d t \cdot \Delta S \cdot n=n v \Delta S d t$, where $v$ is the atom velocity, $n$ is the atom number density.

$$
R=n v \cdot \frac{m}{\rho_{N b}}
$$

so we have atom number density, $n=\frac{R \cdot \rho_{N b}}{m \cdot v}$

Next, we estimate the equivalent partial pressure, which is compared with Argon plasma in the next chapter.

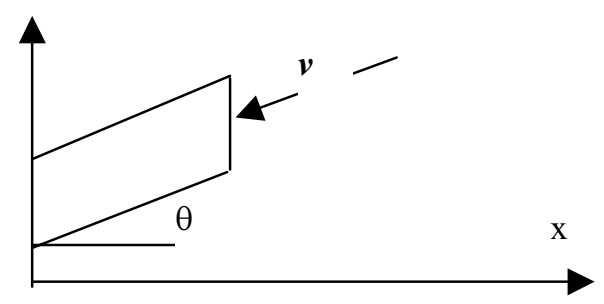

Fig. 3-15 The illustration for partial pressure computation

Again, consider a small $\Delta \mathrm{S}$, we have impact $d I_{\mathrm{i}}$ of $n_{i}$ atoms per unit volume with speed $v_{i}$ is

$$
2 \cdot\left(m v_{x i}\right) \cdot\left[\Delta S \cdot\left(v_{x i} d t\right)\right] \cdot n_{i}
$$

Considering that half of those $n_{i}$ atoms will not impact the imaginary wall, the total impact is

$$
d I=\frac{1}{2} \sum_{i} 2 n_{i} m v_{i x}^{2} \Delta S d t
$$

and the pressure is

$$
P=\frac{F}{\Delta S}=\frac{d I}{d t} \frac{1}{\Delta S}=\sum_{i} n_{i} m v_{i x}^{2}=m n \overline{v_{x}^{2}}
$$

Niobium's melting point temperature is $2468{ }^{\circ} \mathrm{C}$. We can assume the niobium atom flies out with $0.2 \mathrm{eV}$ thermal kinetic energy, with a corresponding speed of about $630 \mathrm{~m} / \mathrm{s}$. If the deposition rate is $75 \AA / s$, then the niobium atom number density is 


$$
n=\frac{R \cdot \rho_{N b}}{m \cdot v}=6 \times 10^{17} / m^{3}
$$

Assuming the $\mathrm{Nb}$ atoms are uniformly distributed, the distance between each atom is about $1.3 \times 10^{-6} \mathrm{~m}$.

If electron velocity is $10^{6} \mathrm{~m} / \mathrm{s}$, which is considered quite modest, the cycling radius in a 875 Gauss magnetic field is $R=6.5 \times 10^{-5} \mathrm{~m}$. It is about 50 times larger than the niobium atom separation distance. That means even if the high magnetic field makes the electron cycle, the electron is essentially traveling straight in the $\mathrm{Nb}$ atom matrix. This simplifies the calculation of the electron neutral collision rate, which is simply

$$
v=n_{0} v_{e} \sigma_{0}
$$

$\sigma_{0}=\pi \mathrm{a}_{\mathrm{o}}^{2}, a_{0}=\frac{h^{2}}{m_{e} e^{2}}, h$ is Planck's constant. $a_{o}$ is picked using this formula, for it is closest to experimental data for electron neutral collisions [49].

$$
\sigma_{0} \sim 3 \times 10^{-15} \mathrm{~cm}^{2}, v=1.335 \times 10^{5} \mathrm{~Hz}
$$

which means the average time before an electron hits the niobium atom is $7.5 \times 10^{-6}$ second. Since elastic collisions result in little electron energy loss, the more interesting cross section is the ionization cross section.

\section{Ionization cross section for $\mathrm{Nb}$}

The ionization cross section of niobium can be approximately computed using the BinaryEncounter-Bethe model. [50]

$$
\sigma_{B E B}=\frac{S}{t+u+1}\left[\frac{Q \ln t}{2}\left(1-\frac{1}{t^{2}}\right)+(2-Q)\left(1-\frac{1}{t}-\frac{\ln t}{1+t}\right)\right]
$$

where $\mathrm{t}=\mathrm{T} / \mathrm{B}, \mathrm{u}=\mathrm{U} / \mathrm{B}, \mathrm{S}=4 \pi \mathrm{a}_{0}{ }^{2} \mathrm{~N}(\mathrm{R} / \mathrm{B})^{2}, \mathrm{~T}$ is incident electron energy, $\mathrm{B}$ is $\mathrm{Nb}$ electron binding energy for expected ionization electron, $U$ is the electron orbital kinetic energy, $a_{0}$ is Bohr radius $0.52918 \AA$, $\mathrm{R}$ is Ryderburg constant $13.6057 \mathrm{eV}$, and $\mathrm{Q}$ is the dipole constant. 


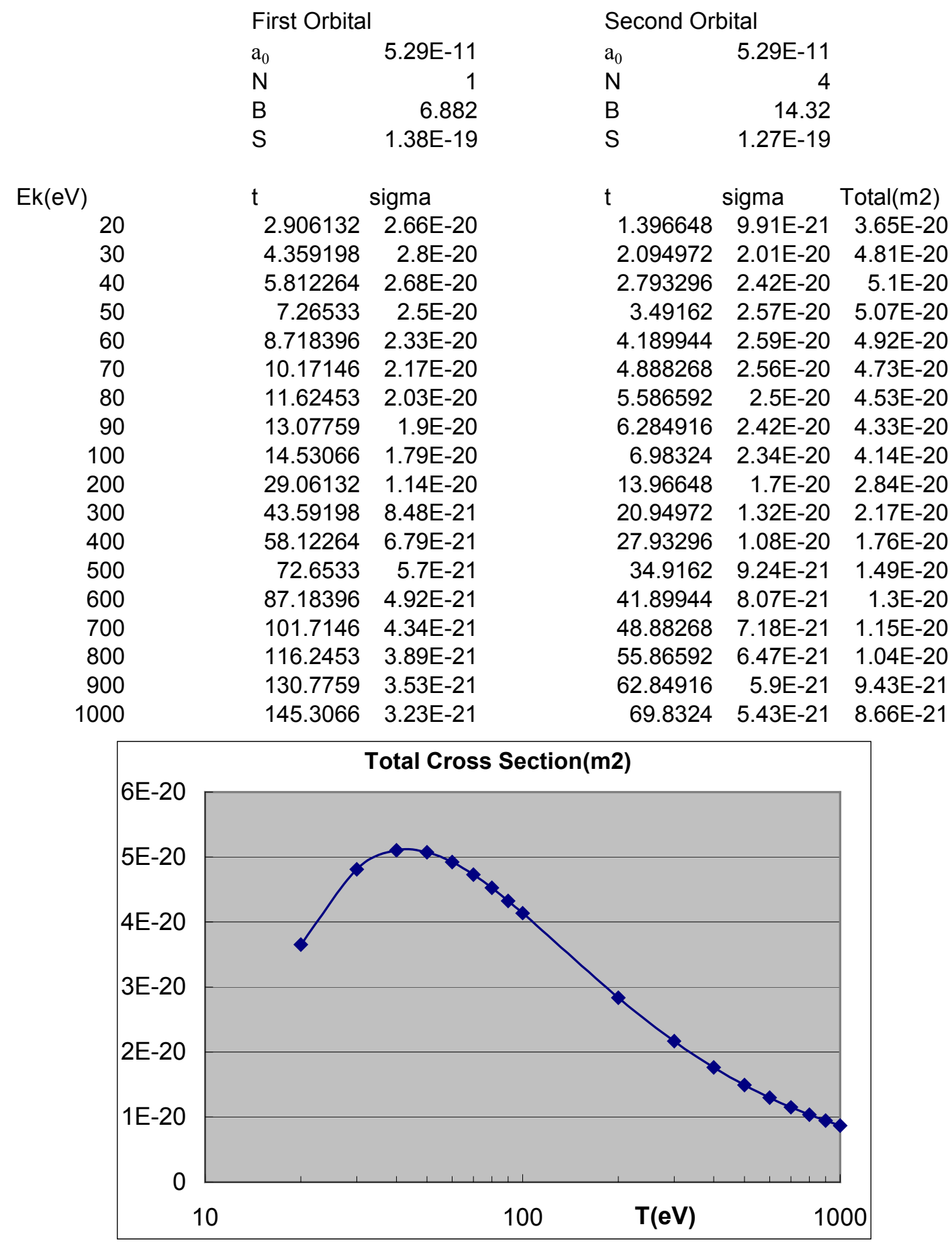

Fig. 3-16 Niobium total ionization cross section for the 5 outer shell electrons.

This spread sheet shows the estimation from BEB model. 
In a rough approximation, $\mathrm{Q}$ can be replaced by 1 , and $\mathrm{u}$ by 0.5 from the simple example in the hydrogen atom. The total ionization cross section is the sum of all the niobium electrons. As a further approximation, consider only the one $5 \mathrm{~s}$ and four $4 \mathrm{~d}$ electrons. The spreadsheet in figure 3-16 shows the computed data without experimental comparison.

The above ionization cross section may underestimate the cross section happening inside the plasma. The ionization process is more complicated inside the low temperature plasma. The Penning process (if it is significant) and ionization of the metastable $\mathrm{Nb}$ atoms also contribute to the effective ionization rate of the plasma. So far, there is no such published data available for niobium.

For comparison purpose, $\mathrm{H}$ atoms have higher maximum ionization cross-section of $7 \times 10^{-17}$ $\mathrm{cm}^{2}$ around $52 \mathrm{eV}$ electron energy.

\section{Off-resonant ECR for better ionization}

According to the spreadsheet estimation in Fig. 3-16, the ionization will likely happen with 30-100 eV electrons. Based on the collision rate analysis, we are able to simulate the electrons movement inside the ECR magnetic field.

The simulation shows that a little off-resonant ECR field accelerates the electrons to the exact energy range to achieve the larger ionization cross section. As shown in figure 3-17, the 2.45$\mathrm{GHz}$ RF power coupled with magnetic field of 800 Gauss accelerates and de-accelerates electrons to have their kinetic energy oscillate between $40-100 \mathrm{eV}$. That way, we also maximize the electron's life time by not accelerating the electron too fast thereby causing it to fly out of the ECR region within the mean free path (or within the average life time). As we shall see in chapter 4 , there are two magnetic field values at which we can excite the plasma. They are both about 70 gauss away from the exact ECR resonant field, 875 Gauss.

One has to note that if the electron mean free path decreases following increasing atom density, off-resonant ECR may not be able to accelerate electrons to appropriate energy range. 


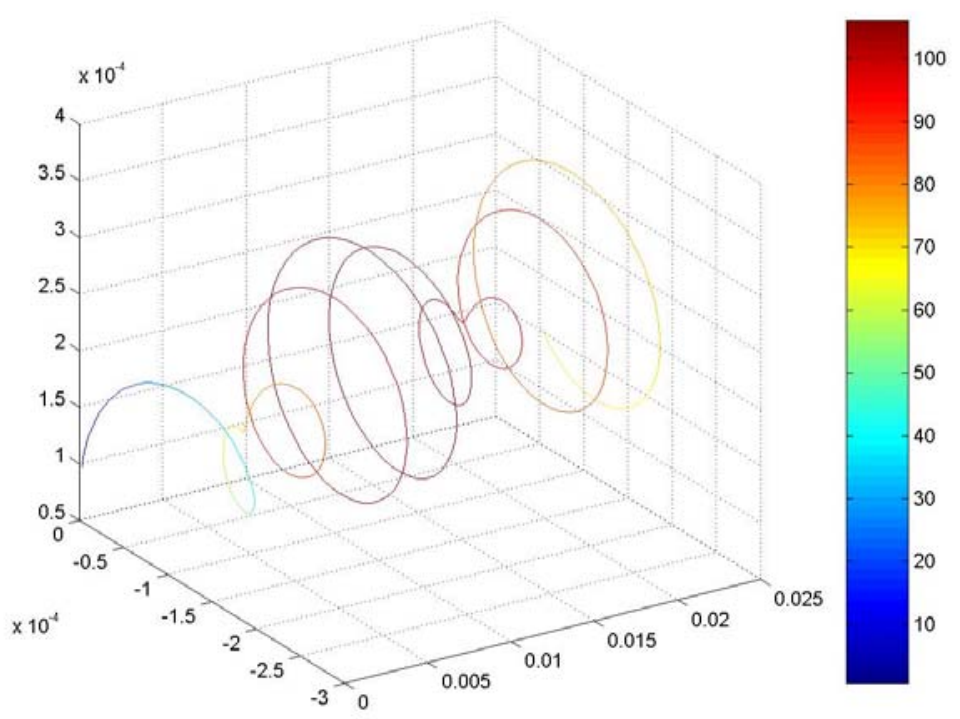

Fig. 3-17 A typical electron's movement inside the ECR-magnetic field coupled with the longitudinal electric field. Color information denotes the electron kinetic energy in $\mathrm{eV}$, axis unit is meter.

\section{3-7. Retarding field beam energy analyzer}

To characterize the energy distribution of the ion beam to the substrate, a retarding field beam analyzer is developed. The idea is close to what Bohm and Perrin have done [51], redesigned to make it easy to handle and cheap to make, and tailored to best suit the energetic niobium ion beam.

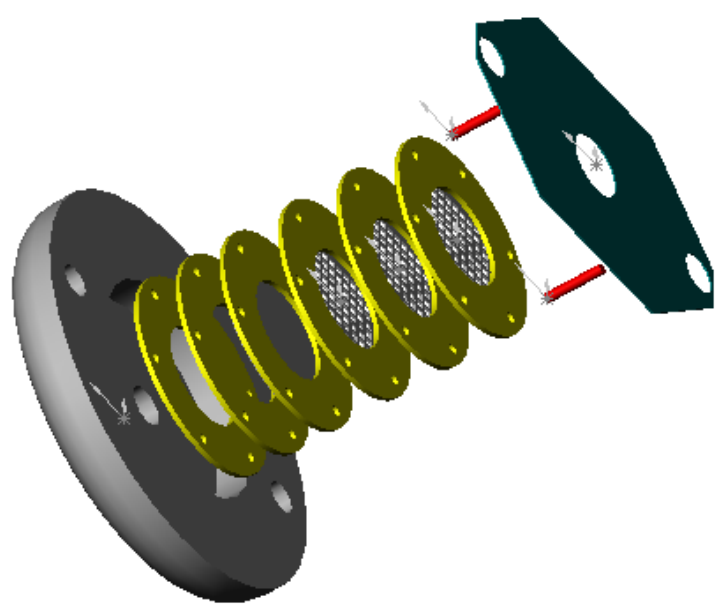

Fig. 3-18 The assembly view of the retarding field beam analyzer. 
The beam analyzer incorporates three grids for retarding field control, one entrance grid and one collector. All of these are enclosed inside the substrate holder as illustrated in the assembly view in figure $3-18$.

\section{The Ion Energy Analyzer}

The original design by Bohm and Perrin requires the analyzer to be as short as $0.82 \mathrm{~mm}$ and is designed to measure the plasma ion energy with the least disturbance to the plasma itself. The authors tried to simplify the original design to make it really low cost and easy to assemble and easy to operate. Although there is plasma disturbance due to the enlarged size of the analyzer, it is accurate enough to measure the ion beam energy as is demonstrated later.

By making the size larger, the parts could be handled easily, thus reducing the manufacturing cost, and reducing the assembly time. The analyzer uses macor ceramic as insulators. They are made by drilling holes through ceramic rod, then cutting the rod into thin pieces with a diamond saw. Wire mesh is used as the electrostatic potential grid. The wire mesh is made of 0.0065 -inch diameter Stainless Steel wire with 620 micron opening square holes and has $60 \%$ transparency. The assembly view is shown in figure 3-18. The top cover can also accommodate on additional grid instead of plain holes to reduce the plasma disturbance. Two ceramic poles act as alignment aids to keep grid holes in sync and keep ceramic insulators in place during assembly. The remaining ceramic holes guide the conducting wires to the holder feedthrough. The top cover is screw tightened into the holder to keep everything tight. The holder can be any shape. The chosen shape shown in figure 3-18, allows it to be installed inside the ECR plasma reactor.

\section{The Retarding Field Mechanism}

The grid potential is shown in figure 3-19. The first grid is negative potential relative to the ground. It serves the purpose of stopping the plasma electrons from getting into the beam analyzer. The third grid potential is lower than the collector. It is expected to suppress the secondary electrons from the collector. The second grid is the retarding field grid. It varies from zero to some higher voltage depending on the ion energy to be measured. When the voltage is positive at some level, the ions with lower energy will not travel to the collector, thus the current 
can be monitored to get the I-V curve, which in turn can be used to derive the energy spread of the ion beam.

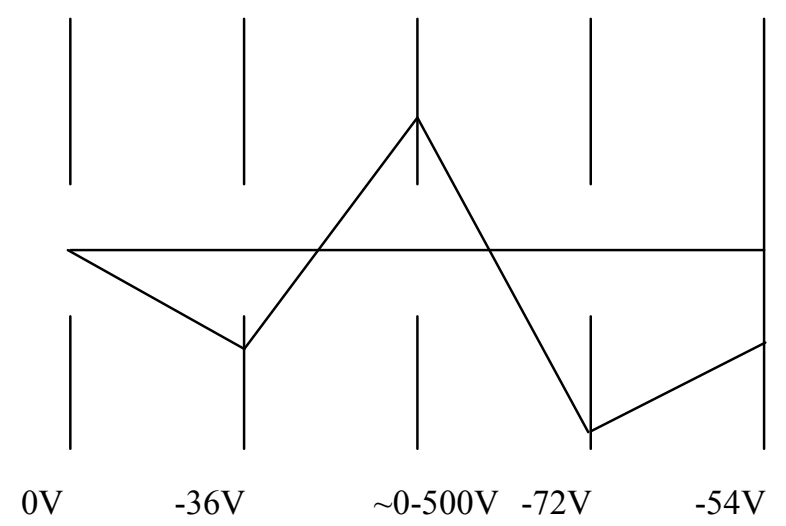

Relative Voltage to the 0V-300V Bias power supply

Fig. 3-19 The grid potential for retarding field ion energy analyzer

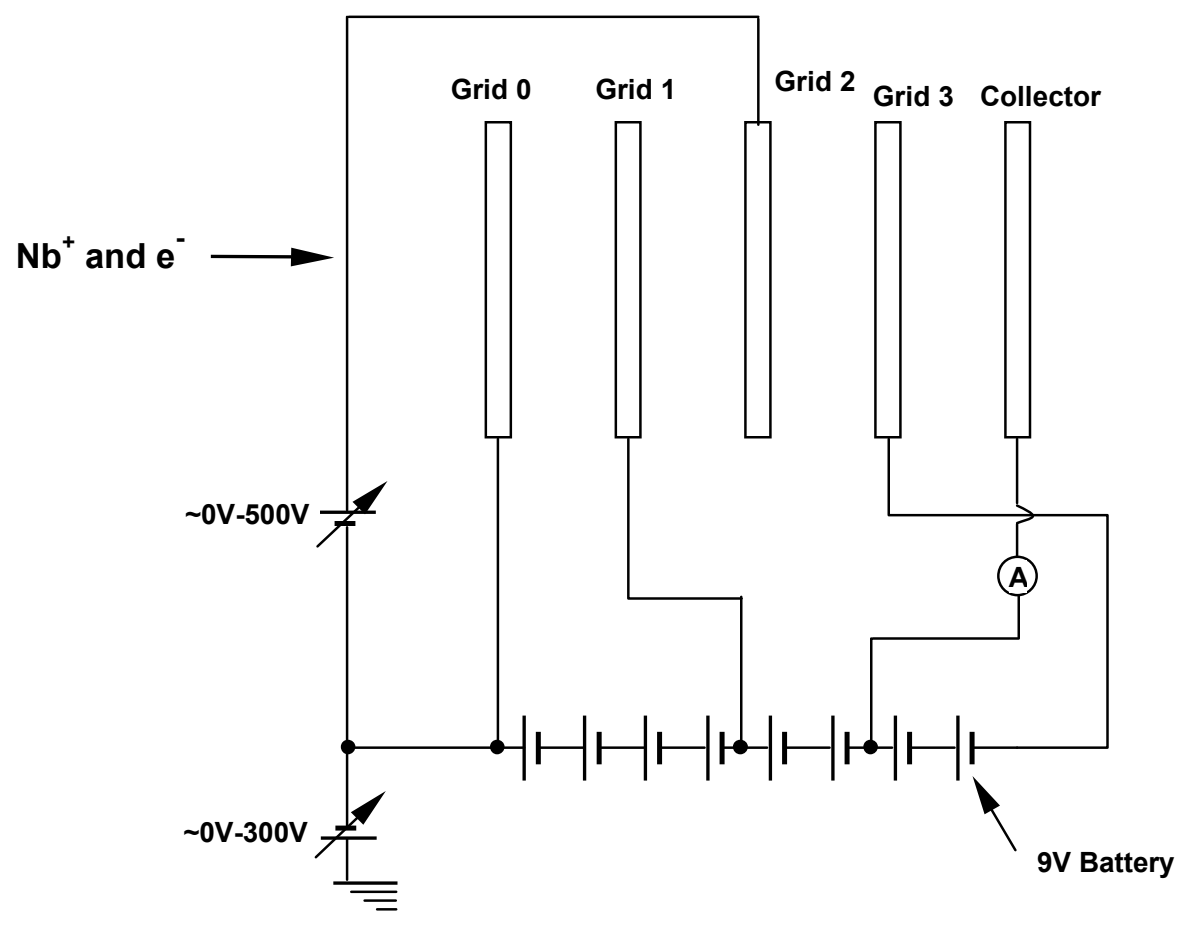

Fig. 3-20 The circuit diagram for retarding field ion energy analyzer 


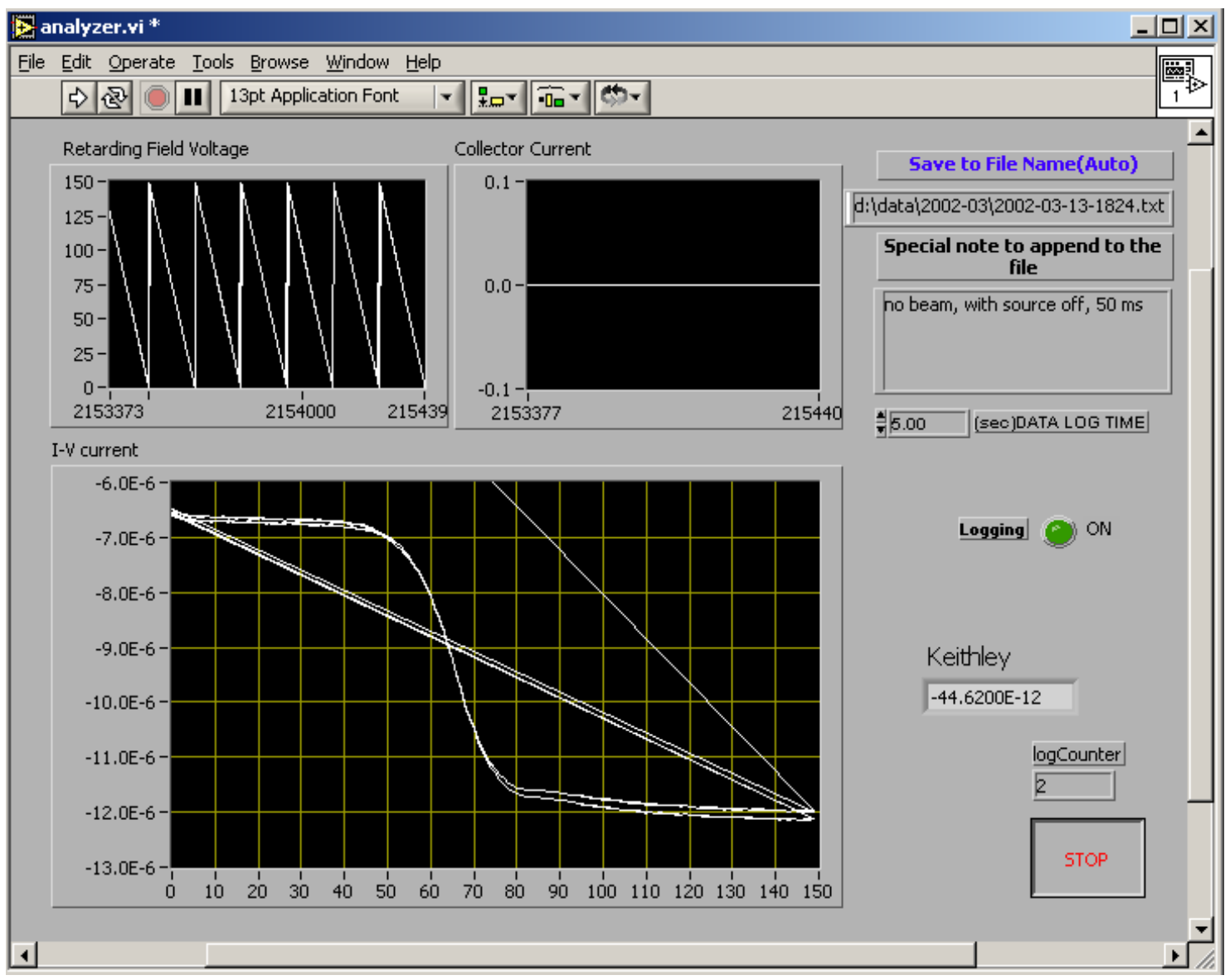

Fig. 3-21 The labview panel screen shot shows an I-V curve during beam test. 
Grid one, three and the collector potentials are provided by eight serial connected nine-volt batteries as displayed in figure 3-20. The retarding field grid potential is provided by Keithley pico-ammeter built in voltage power supply providing $-500 \mathrm{~V}$ to $500 \mathrm{~V}$. The pico-ammeter monitors the collector current and is controlled by a labview program to log the I-V curve. The voltage is incremented at one-volt intervals every $50-\mathrm{ms}$. The Labview panel is shown in figure 3-21. The analyzer itself can be floated below $-300 \mathrm{~V}$.
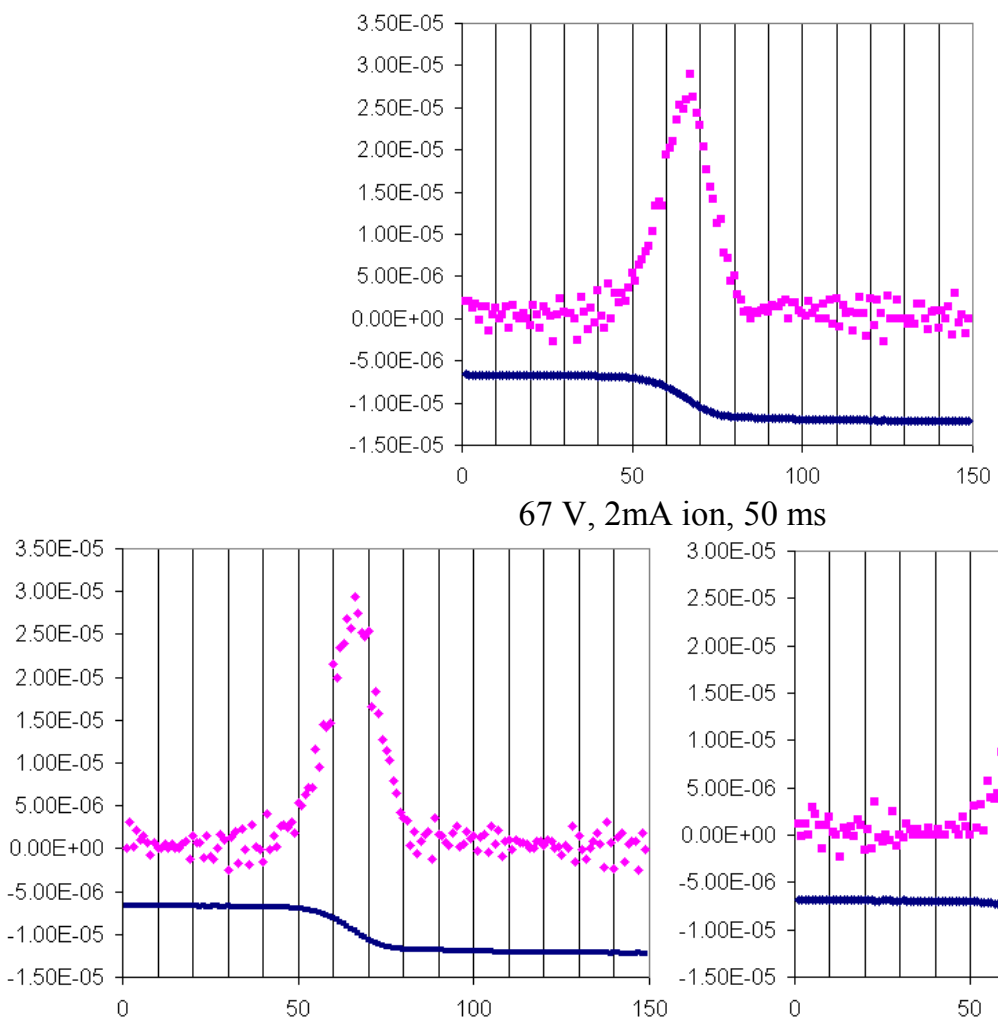

$67 \mathrm{~V}, 2 \mathrm{~mA}$ ion, $200 \mathrm{~ms}$
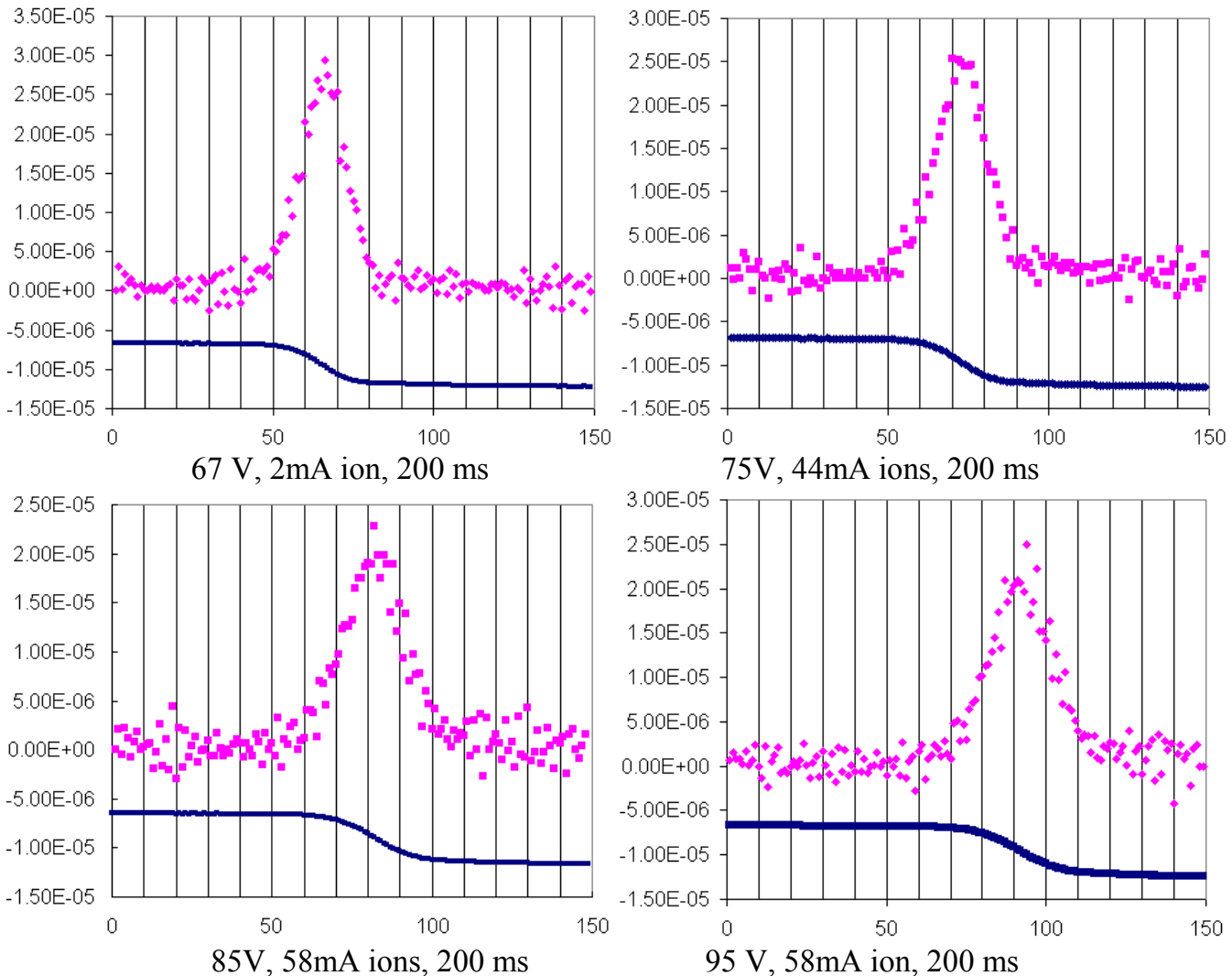

$75 \mathrm{~V}, 44 \mathrm{~mA}$ ions, $200 \mathrm{~ms}$

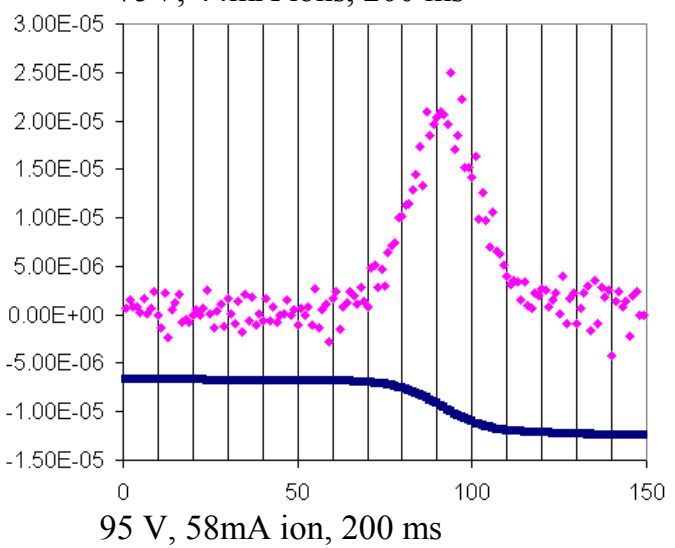

Fig. 3-22 The ion beam energy distributions with different ion beam energies. The peak curve is $\mathrm{dI} / \mathrm{dV}$ derived from I-V curve. 


\section{The Testing with Ion Gun}

The analyzer is tested in the vacuum chamber for ion beams with different energies. The results are in figure 3-22. The red dotted curve is derived from I-V curve, which is proportional to the number of ions with energy in volts denoted on x-axis. The ion gun uses Argon as its working gas; the pressure of Argon inside the chamber is $1.5 \times 10^{-4}$ Torr. During ion gun beam test, the analyzer is at ground potential.

\section{Discussion}

The collector is made of copper foil. The front plate open hole can be variable size. The hole size was increased to $1 / 4$-inch diameter to increase the ion current to the collector, thus increasing the signal to noise ratio. But the increased hole opening allows more ions to get inside of the analyzer. This in return increases the space charge effect inside the analyzer, and changes the ion trajectory, thus increasing the ion energy spread. The negative current level reflects the fact that the electrons from ion gun neutralizer are contributing to the collector current. This effect can be eliminated by increasing the grid one potential. But that is not necessary since the electrons inside the analyzer don't create extra ionization. This is proved by the absence of linear increase of collector current as described in [51]. The electrons from ion gun neutralizer help to reduce the space charge effect of ions inside the analyzer. Overall this effect doesn't affect the ion energy measurement.

\section{3-8. Data acquisition}

A labview data log program is developed to record about 15 parameters during system operation. The data acquisition system serves 4 direct controlled IEEE instruments and 3 power supplies using a DAQ board to log their voltages and currents. 


\section{Chapter 4. Experiment with Argon, Niobium Vapor and Copper Vapor}

\section{4-1. Overview}

The system is tested for copper and niobium vapor. With nitrogen and argon, the plasma reaction is quite well established, since the gas molecules move inside the circular waveguide in every direction, filling the entire volume. The copper or niobium vapor moves in a fixed direction, traveling with higher velocity due to the electron beam heating. To achieve the plasma reaction, the equivalent partial pressure of the metal vapor needs to be higher than that of Argon gas. The minimum argon pressure to sustain the plasma reaction is $1.1 \cdot 10^{-5}$ Torr. While the copper and niobium partial pressure is about $1.0 \times 10^{-4}$ Torr, which comes from metal flux of $65 \AA / s$ for copper and $100 \AA / s$ for niobium.

While there is a minimum metal flux requirement for plasma ignition, the RF power plays the main role to change the ionization rate. In the current system, a moderate 330 watts RF power is used to feed into the plasma reaction. Typically, niobium plasma consumes about 240 watts RF power on a $132 \AA /$ s flux. The neutral flux rate drop of $65 \AA / s$ on the thickness monitor represents around $50 \%$ ionization rate. If one sixth of all the niobium ions are assumed extracted, the substrate deposition rate would be $11 \AA /$ s. The maximum ion extraction is observed when the substrate collection current remains unchanged $(34 \mathrm{~mA})$ with $-12 \mathrm{~V}$ bias. In this chapter, I will describe some of the parameters influencing plasma operations, which are relevant to the deposition.

\section{4-2. Absorbed RF power and the ECR magnetic field}

According to the discussion in chapter three, there were two different magnetic field values for plasma ignition. The first test to ionize the niobium beam revealed exactly the phenomenon of off-resonant ECR plasma ignition. Table 4-1 lists the absorbed RF power versus the current of the magnetic coil for niobium. 
It is worth pointing out that this two-field plasma ignition was not observed in the argon test when argon pressure is high and the electron mean free path is much lower.

Table 4-1 The absorbed RF power and the current level of second current source

\begin{tabular}{|c|c|}
\hline $\begin{array}{c}\text { Second Current } \\
\text { Source }(\mathrm{A})\end{array}$ & RF Absorption $(\mathrm{W})$ \\
\hline 50 & 5.2 \\
135 & 8.7 \\
138 & 8.8 \\
145 & 9 \\
151 & 10 \\
151 & 100 \\
156 & 9.7 \\
165 & 9.1 \\
180 & 7 \\
200 & 5 \\
& \\
250 & 6 \\
270 & 8 \\
280 & 10 \\
298 & 12 \\
298 & 110 \\
300 & 11.8 \\
320 & 8 \\
350 & 5 \\
\hline
\end{tabular}

First current source is at $1134 \mathrm{~A}$

\section{4-3. RF power, evaporation power and the plasma}

While there is no systematic test to understand how the RF power and evaporation power affect the ionization and niobium ion beam, the recorded data for many runs of the system does reveal some interesting correlations between RF power, evaporation power and the plasma operation. The thickness monitor for the neutral niobium beam doesn't give a stable reading for the niobium flux with high deposition rate. The collector current measured by substrate-holder bias power supply does provide stable current readings.

Based on the system running data, it is found that within the same evaporation power, or roughly the same niobium neutral flux, increasing RF power level increases the rate of niobium ionization in terms of collector current, until the RF absorption is maximized. That is shown in figure 4-1. 


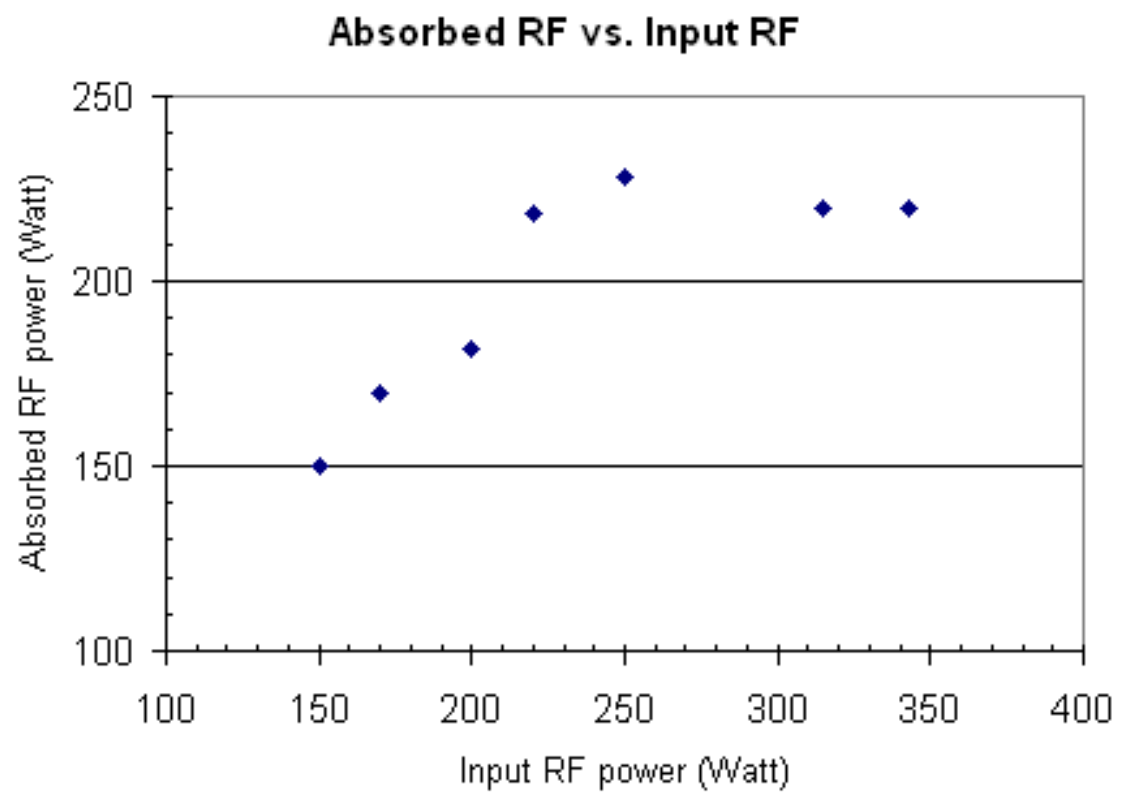

Fig. 4-1 Absorbed RF power saturates as RF input power increases

For most plasma operations, the collector current will be directly affected by the absorbed RF power whether the absorption is saturated or not. As shown in fig. 4-2, the collector current varies almost linearly as a function of increasing RF absorption.

\section{Collector Current}

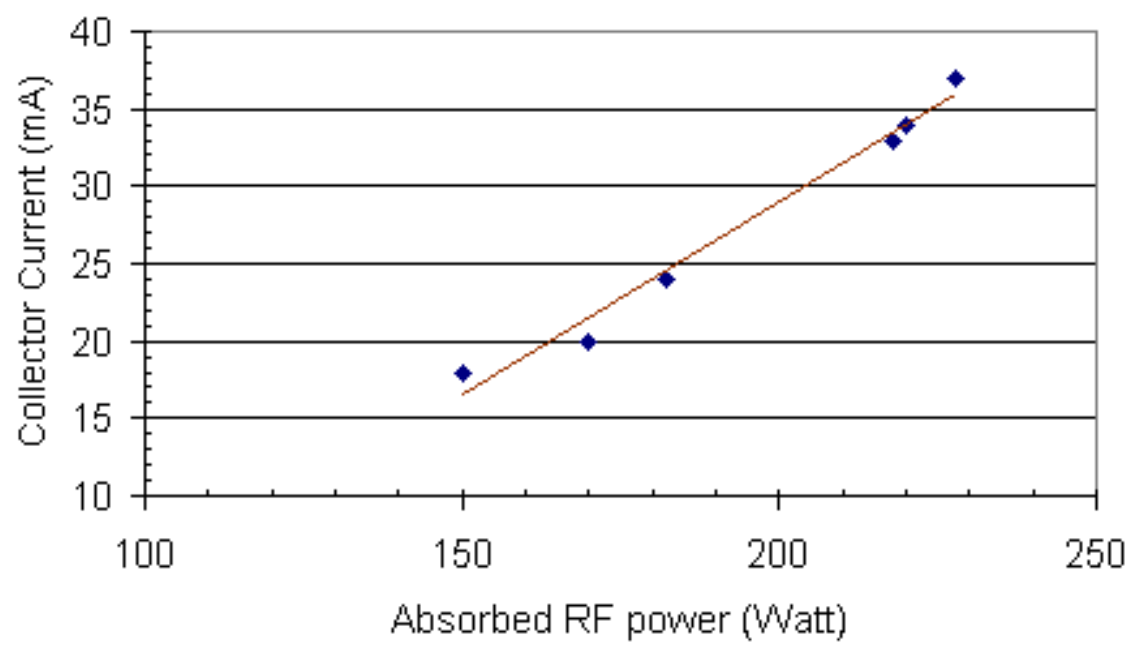

Fig. 4-2 Collector current is almost linear to the absorbed RF power

During plasma operation, the increased beam power increases the niobium evaporation rate. 
As more RF power is absorbed in the plasma, more ions are created to reach the collector. This is observed in several plasma operations. Typical data is listed in table 4-2. Again, there is no systematic running of the system to investigate this effect; so data from separate runs are used. The beam power is not directly recorded since the change of the power supply reading is within the variations for different runs. Also for each single operation, the niobium target is a different shape. That causes the unique correlation between beam power and the niobium evaporation rate. Even though the thickness monitor is not showing stable readings, it nevertheless shows the increased niobium neutral flux as shown in table 4-2.

\section{Table 4-2 Increased Beam Power and the RF absorption}

\begin{tabular}{|c|c|c|c|}
\hline RF Input (W) & RF absorb (W) & Current (mA) & Rate $(\AA / s)$ \\
\hline 343 & 220 & 34 & 46 \\
338 & 270 & 36 & 50 \\
316 & 300 & 40 & 95 \\
\hline
\end{tabular}

In conclusion, if the deposition rate is directly proportional to the collector current, then adjusting the beam power or simply adjusting the RF input power will change the deposition rate at the substrate at the specific bias voltage, as long as the substrate is below the $-12 \mathrm{~V}$ bias voltage.

\section{4-4. Measurement of niobium ion energy at the biased substrate}

During the niobium plasma measurement, the ion analyzer is floated inside the plasma at certain bias voltages through a separate power supply. When the bias voltage power supply is in operation, extra noise from E-beam gun power supply is coupled into the ion analyzer circuit, which adds noise to the $\mathrm{d} / \mathrm{dV}$ data. It is found that the ion velocities didn't follow the Maxwelian distribution at the substrate position. In fact the I-V data can be best fitted with the formula:

$$
I=I_{0}+\frac{a}{1+e^{-\frac{V-V_{0}}{b}}}
$$

$a, b, V_{0}$ and $I_{0}$ are fitted parameters. 


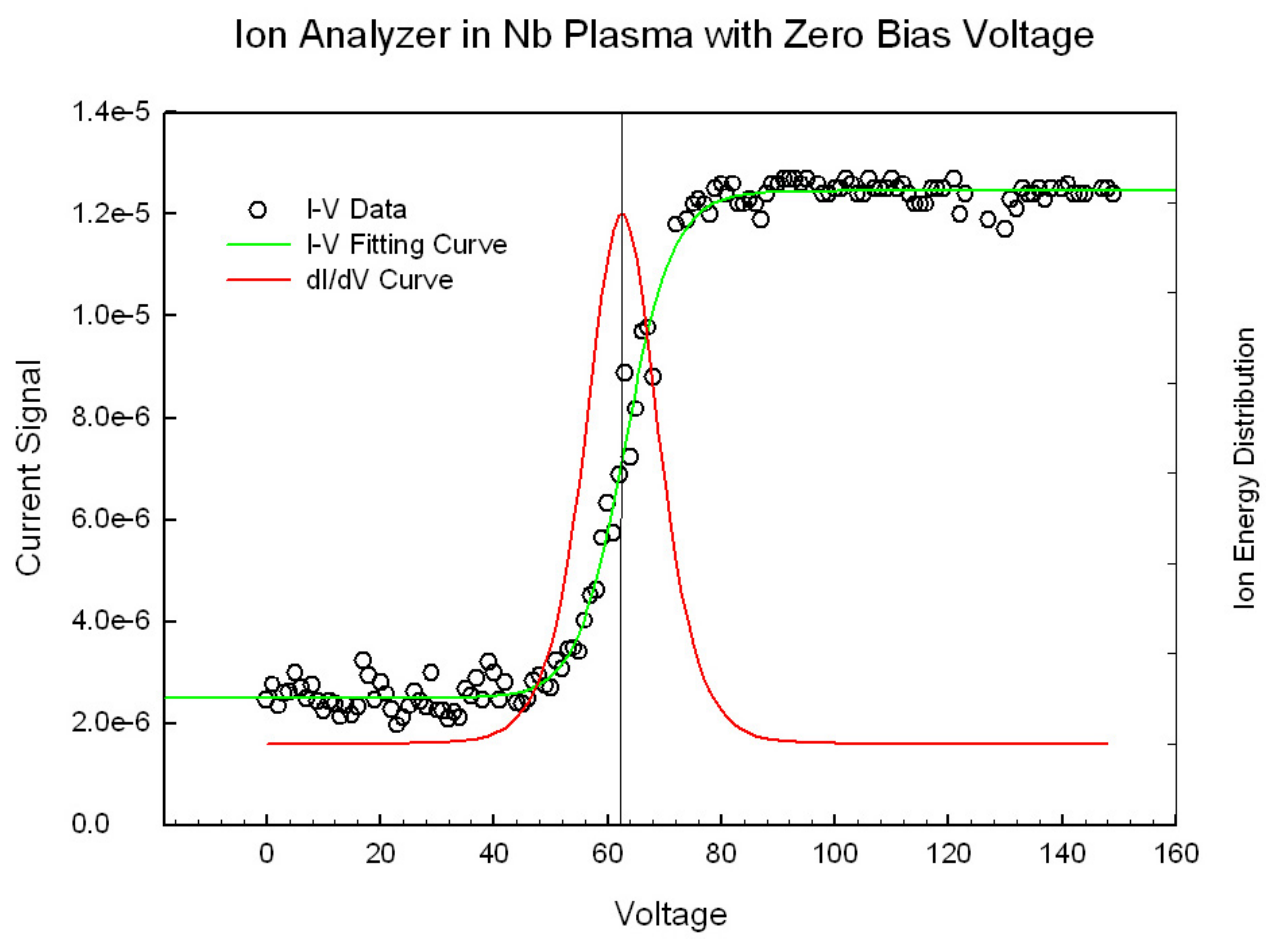

Fig. 4-3 Niobium ion kinetic energy distribution measured at substrate holder

Ion Analyzer in Nb Plasma with -33V Bias Voltage

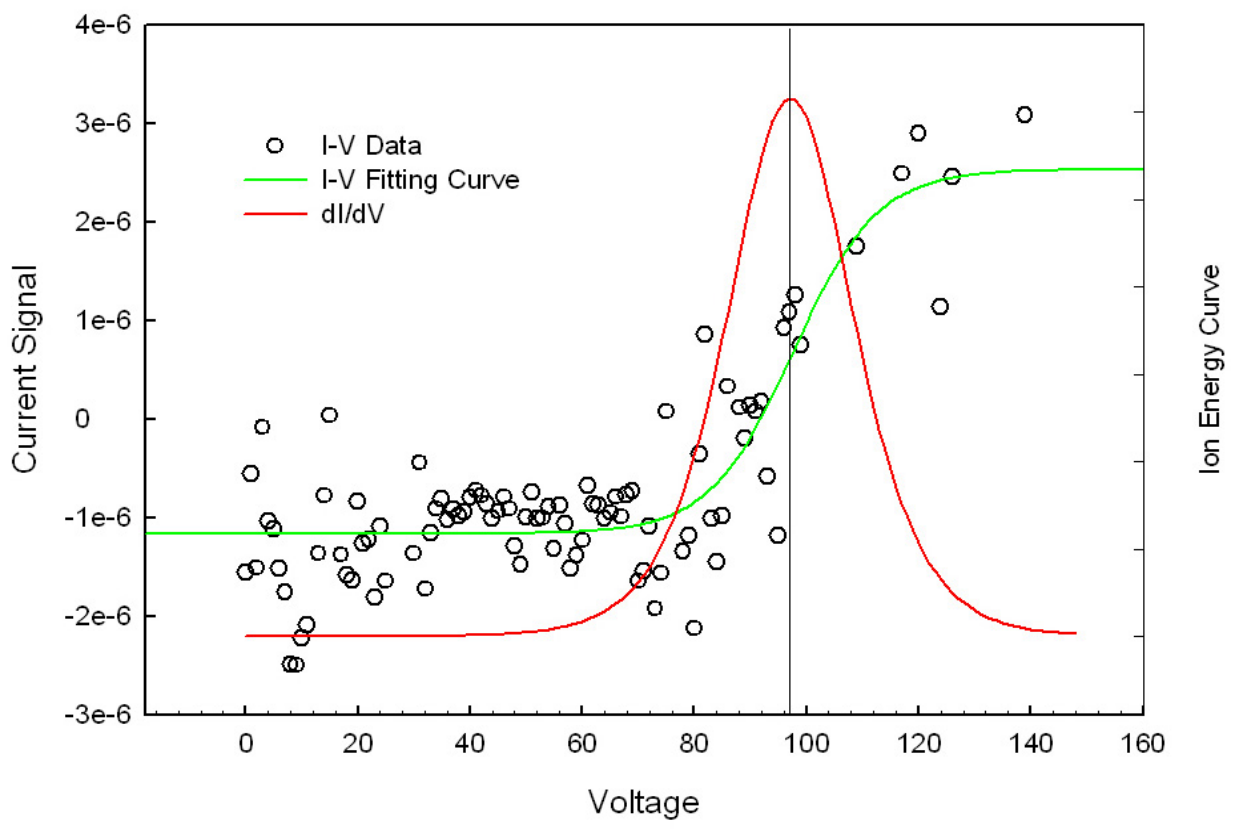

Fig. 4-4 Niobium ion kinetic energy distribution when substrate holder is biased at $-33 \mathrm{~V}$ 
Ion Analyzer in Nb Plasma with -51V Bias Voltage

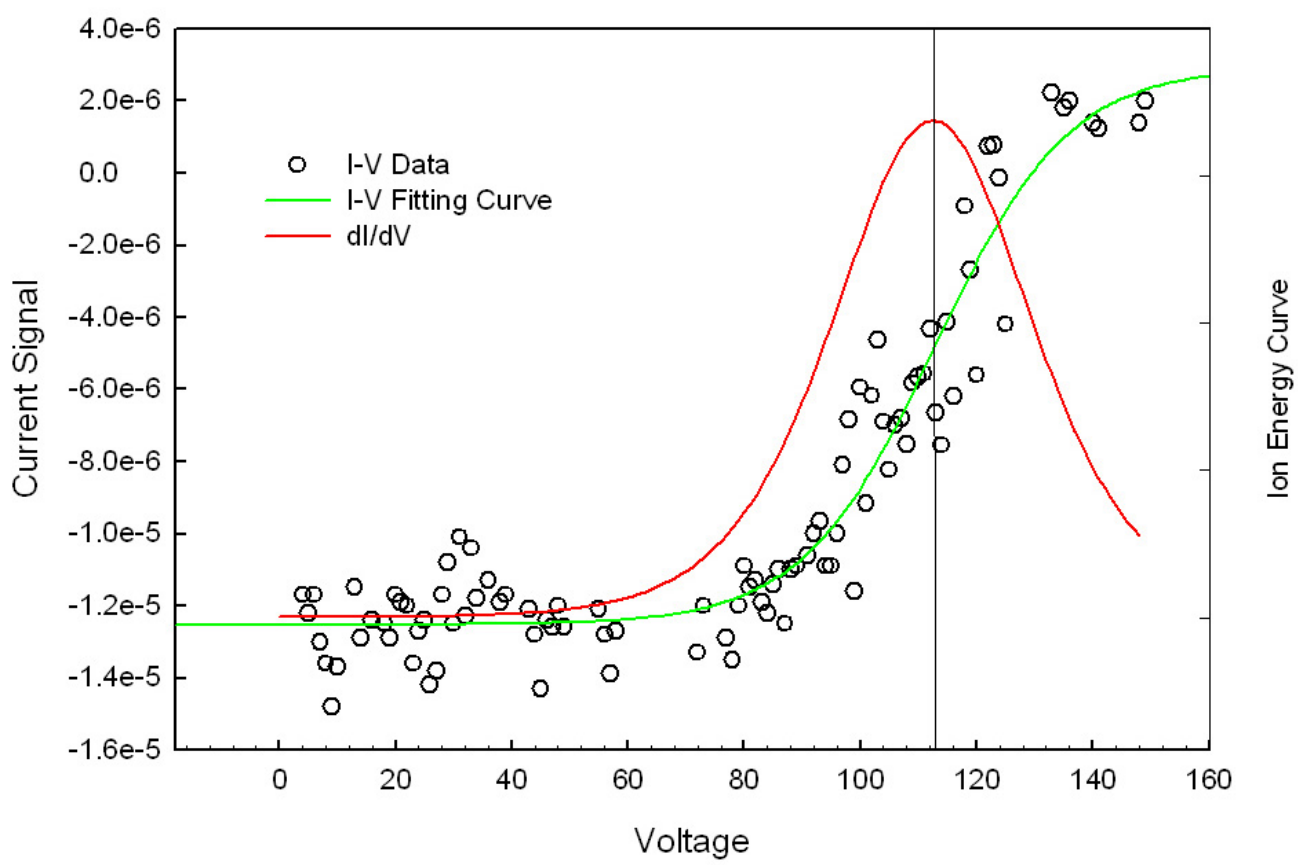

Fig. 4-5 Niobium ion kinetic energy distribution when substrate holder is biased at $-51 \mathrm{~V}$

Ion Analyzer in Nb Plasma with -84V Bias Voltage

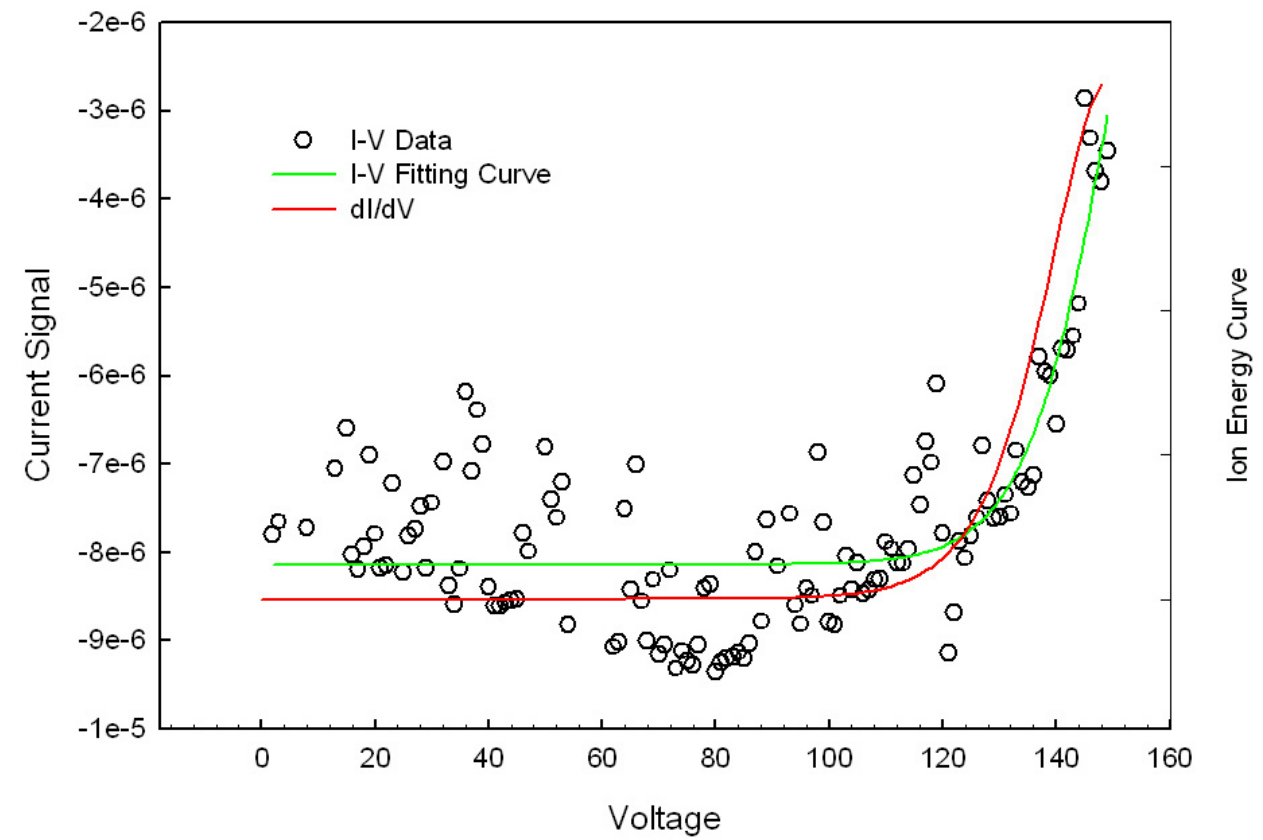

Fig. 4-6 Niobium ion kinetic energy distribution when substrate holder is biased at $-84 \mathrm{~V}$ 
The plasma operating condition can be described as input RF power at 250 Watts, absorbed RF power at 230 Watts (as indicated by the directional coupler). The substrate holder has a current of around $36 \mathrm{~mA}$. Some plasma quenches were observed during the measurement.

The niobium neutral flux of $120 \AA / \mathrm{sec}$ represents a low-pressure unidirectional niobium vapor, and the ions of the plasma can be assumed singly charged. As the fittied results show in fig. 4-3, the niobium ions of the plasma have median energy of $64 \mathrm{eV}$, and the energy spread is around 20 eV. As shown in fig. 4-4 through fig. 4-6, when the bias voltage is added, the ions have on average the added kinetic energy, while the energy spread is increased as a function of increasing bias voltage.

Also observed during the energy distribution measurement, the substrate collector current saturates for a bias voltage below -12 volts. As a result, for substrate biased below $-12 \mathrm{~V}$, the deposition rate would be proportional to the collector current, if re-sputtering of niobium is not significant.

\section{4-5. Deposition Energy versus Deposition Rate}

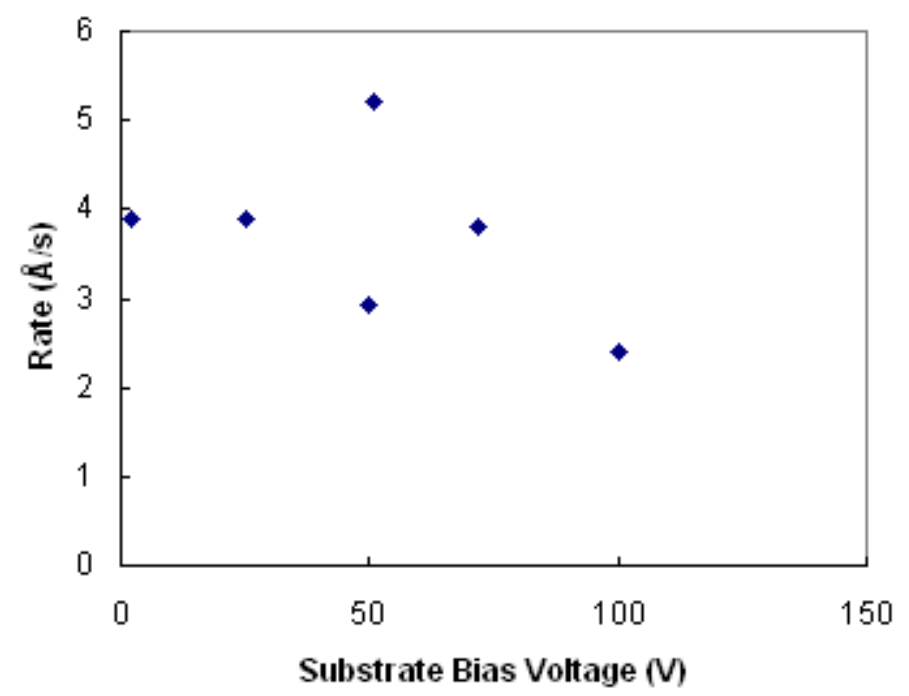

Fig. 4-7 Niobium deposition rate on sapphire versus substrate bias voltage 
It is interesting to note that the re-sputtering of niobium on sapphire is not as significant as originally thought. Figure 4-7 shows that the rate drop is tolerable when the substrate is biased at $-100 \mathrm{~V}$, equivalent to $164 \mathrm{eV}$ niobium ion energy. 


\section{Chapter 5. The Characterization Procedures of Niobium Thin Film}

This chapter describes the parameters characterizing the niobium thin film and the relative techniques or devices to measure these parameters. The results associated with different deposition energies using these measurement techniques are discussed in chapter 6 . The $\mathrm{T}_{\mathrm{c}} / \mathrm{RRR} / \mathrm{B}_{\mathrm{c}}$ measurements are developed in-house. The others are commercial surface analysis tools.

\section{5-1 The transition temperature}

\section{Introduction}

A common induction coil is used to measure the transition temperature of niobium thin film. The induction coil method proves to be one of the easiest ways to characterize a large area superconducting (SC) thin film sample. It provides the overall film quality compared with the resistance method in terms of the transition, and is much simpler compared with the AC susceptibility method. This section describes the effort to develop a coil set to measure the transition temperature by the AC induction method.

\section{The experimental setup}

The simple measurement system is shown in figure 5-1.

The HP function generator described in figure 5-1 can give $\pm 10 \mathrm{~V}$ sine wave over a wide range of frequencies. The lock-in Amplifier is a Stanford Research SR530 with a sensitivity of $0.01 \mu \mathrm{V}$.

Both of the coils are made out of wire-gauge-38 insulated magnet copper wire with about 1000 turns. Coil dimensions are about $10 \mathrm{~mm}$ long, $10 \mathrm{~mm}$ outer diameter and $4 \mathrm{~mm}$ inner diameter. One coil is a little longer than the other. The longer one is selected as pickup coil, with a resistance of $66.2 \mathrm{ohms}$ at room temperature. The driven coil has a resistance of $63 \mathrm{ohms}$ at 
room temperature. Both coils' resistance drops to around $0.987 \mathrm{ohms}$ once the temperature is below 15 Kelvin. One thousandth ohms of resistance change is observed from 10 Kelvin to 6 Kelvin, when the driven signal is a $2 \mathrm{~V}, 50 \mathrm{~Hz}$ sine wave. A $100-\mathrm{Ohm}$ resistor is connected in series with the primary coil in order to further reduce the current change caused by temperature induced coil resistance change, and to protect the coil.

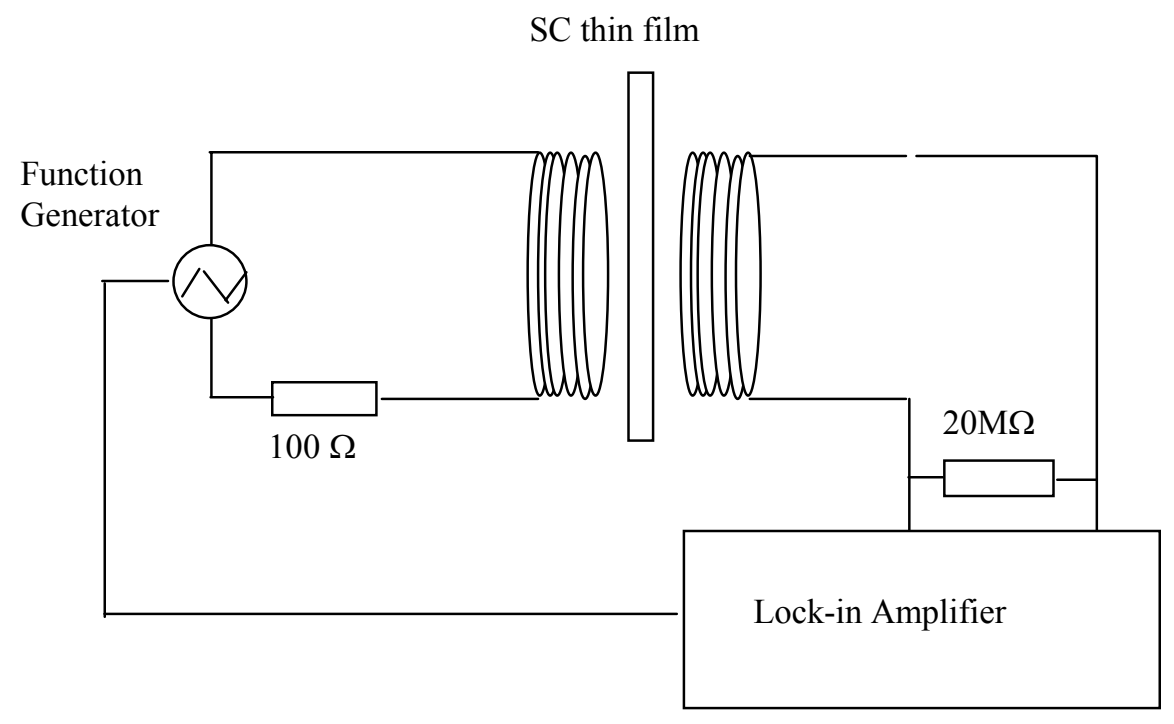

Fig. 5-1 The illustration of the $T_{c}$ measurement

\section{The copper disk magnetic flux shielding}

Due to the copper's high conductivity at low temperature, the copper disk substrate of the niobium thin film shields a large mount of AC magnetic flux created by the driven coil. This is observed by monitoring the pickup coil signal during the cooling down process.
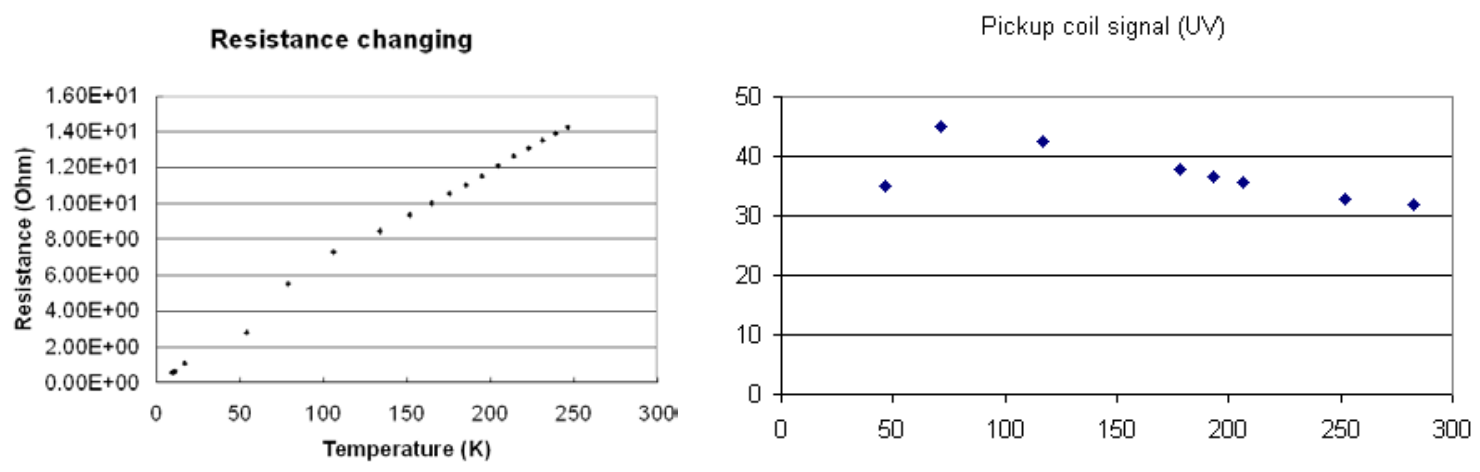

Fig. 5-2 The resistance of a typical copper coil (left chart)

The pickup coil signal, while the primary coil signal maintains the same magnitude of voltage (right chart) 
While the voltage on primary coil remains the same, the decreasing coil resistance should make the coil flux increase as displayed on the left chart of figure 5-2. Even though the pickup coil experienced the same resistance drop, Faraday's law keeps the voltage signal in the pickup coil proportional to the flux it detects. In the right chart of figure 5-2, we see the flux detected by the pickup coil changes only slightly, which reflects the copper disk's increased magnetic field shielding.

\section{Coil self-heating}

By monitoring the pickup coil voltage signal, one should be able to determine whether the coil will exhibit self-heating effects. Since the thermal conductivity decreases as temperature is lowered, and since the coil is tightly wrapped, over heating might be possible at high current. Fortunately, for the primary coil with signal as high as $5 \mathrm{~V}, 50 \mathrm{~Hz}$, there is no observable pickup signal change in 15 minutes duration at 11 Kelvin. That is to say, the resistance of primary coil doesn't change. This suggests the primary coil maintains the same flux magnitude during the $\mathrm{T}_{\mathrm{c}}$ measurement. From table 5-1, we see the quite different thermal conductivity between $7 \mathrm{~K}$ and $11 \mathrm{~K}$. In a separate test, no self-heating is observed below 7 Kelvin.

\section{Table 5-1 The copper thermal conductivity for $R R R=65$}

\begin{tabular}{|c|c|}
\hline Temperature (K) & W/(cm.K) \\
\hline 7 & 7.032315 \\
8 & 8.014042 \\
9 & 8.978411 \\
10 & 9.919377 \\
11 & 10.83126 \\
\hline
\end{tabular}

\section{Edge effect}

Due to the magnetic field enhancement around the sharp edge, the edge effect is unavoidable for superconducting thin films. Magnetic fields perpendicular to the thin film, no matter how low, always cause strong magnetic fields around the edge of the film. The enhanced edge field 
may cause the SC film to lose the SC state. At decreasing temperature, the effective SC film area increases, thus the field shielding capability increases. One should note, in a type II superconductor, the edge may not lose the SC state completely, but the result is the same. The SC film shields more flux as the temperature is lowered. This is quite obvious in both charts in figure 5-3.

\section{$\mathrm{T}_{\mathrm{C}}$}

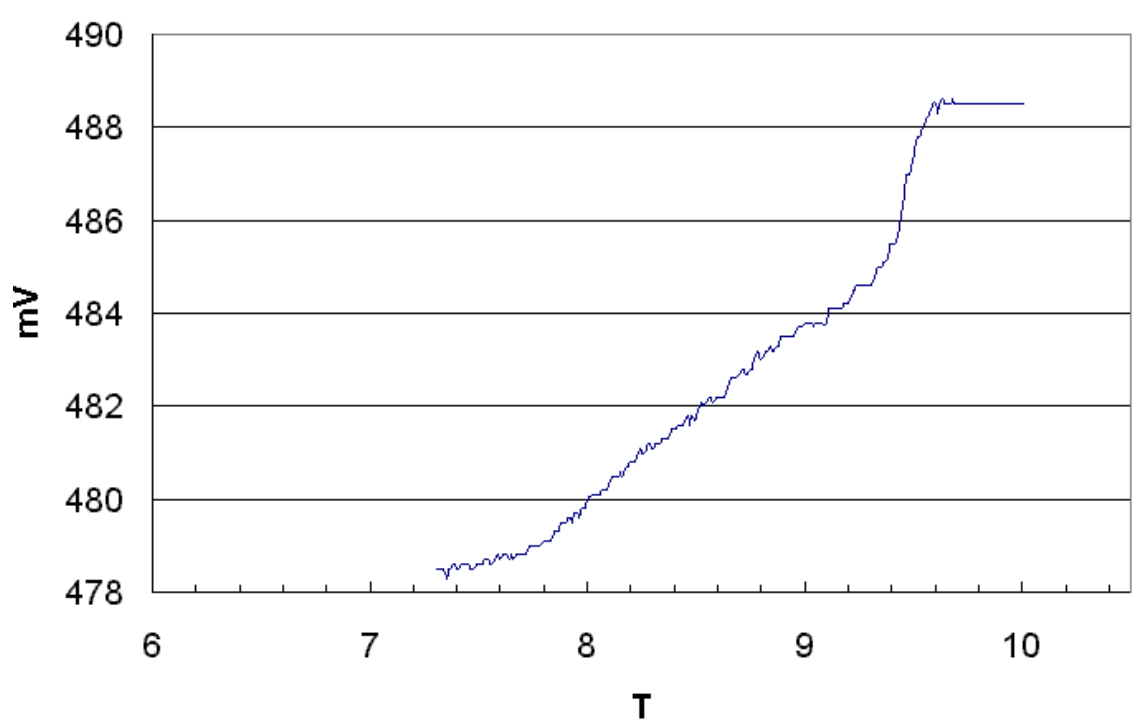

$T_{c}$

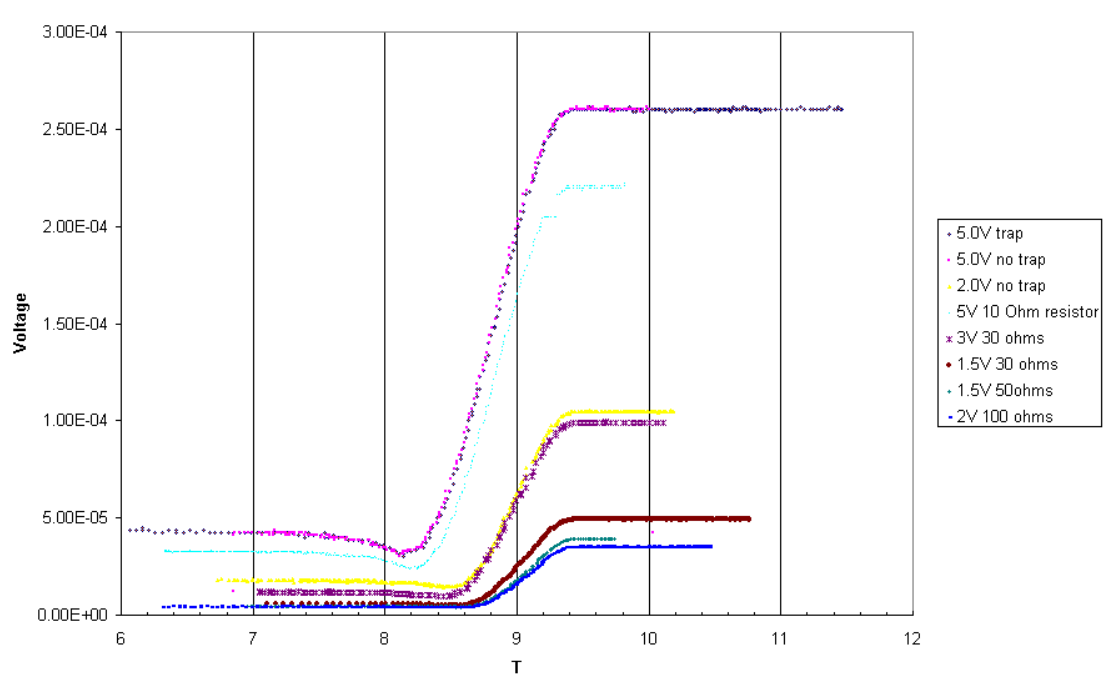

Fig. 5-3 The $T_{c}$ curve measured by a large coil set (upper chart)

The $T_{c}$ curve measured by a small coil set with different driving signals (lower chart)

The temperature in both charts is measured by different diodes, and none of them are calibrated. 
The coils used to obtain the upper chart in figure 5-3 have a size comparable to that of the thin film sample. After reducing the coil size as described at the beginning, the situation is much improved. Also, as the field strength decreases, the transition width narrows. Figure 5-4 is the $T_{c}$ signal curve without much edge effect.

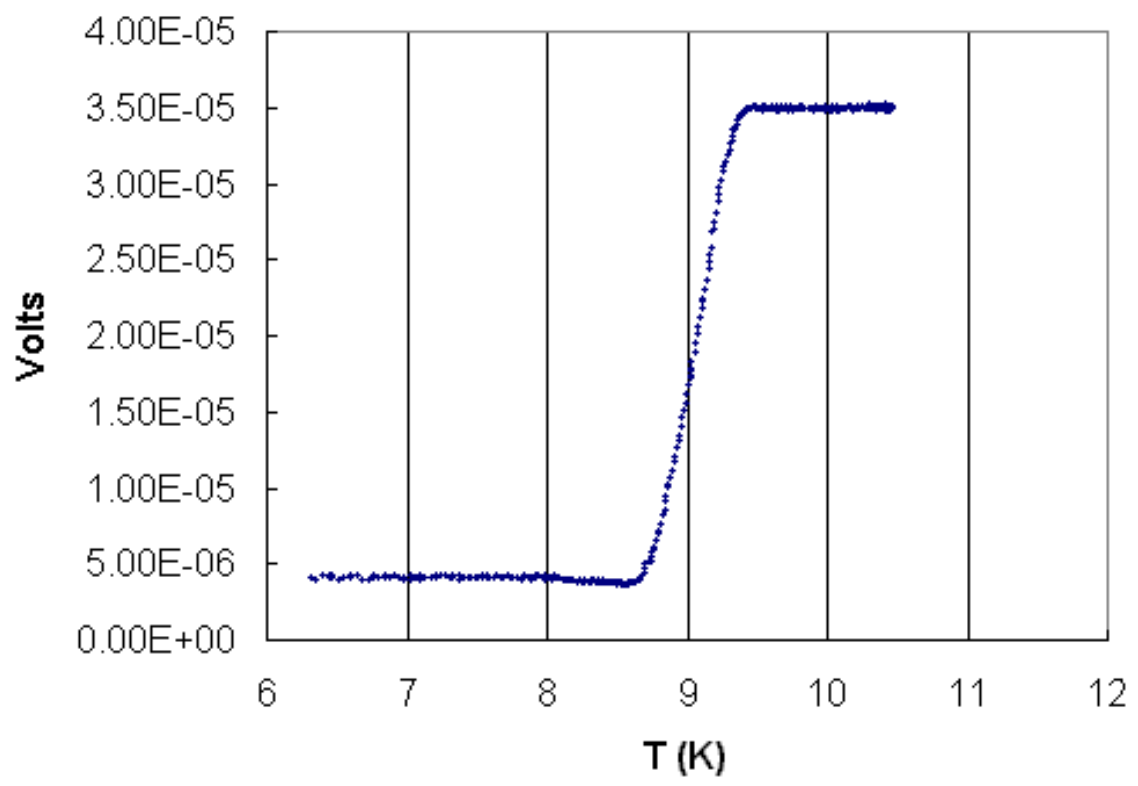

Fig. 5-4 The $T_{c}$ measurement using primary coil signal of $20 \mathrm{~mA}, 50 \mathrm{~Hz}$

The lower end of the transition curve shows a lowered pickup coil signal, which we interpret as the result of superposition from stray fields with a different phase during SC phase transition. This is consistent with the phase information curve in figure 5-5. The transition has started long before the voltage signal starts to show the change. A closed copper can coated with $\mathrm{NbTi}$ or any other superconductor with higher transition temperature would be the best container for the pickup coil.

\section{Conclusion}

One should use as low as possible magnetic flux during $T_{c}$ measurement while not compromising data accuracy; and using an extra shield is expected to improve the measurement. The phase information of SC transition may provide more useful information about the 
transition. The phase transition curve reflects the real transition of SC thin film if no extra shield is used to decrease the stray field effect.

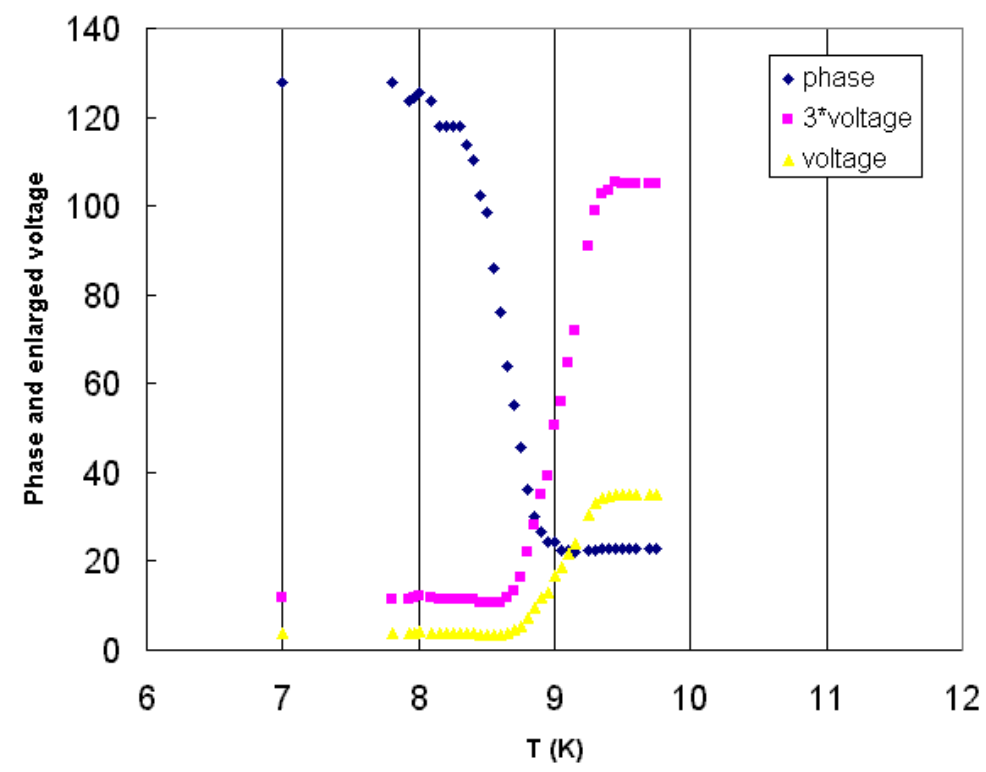

Fig. 5-5 The illustration of the $T_{c}$ measurement with phase information

Tc for Sample 07-22-01

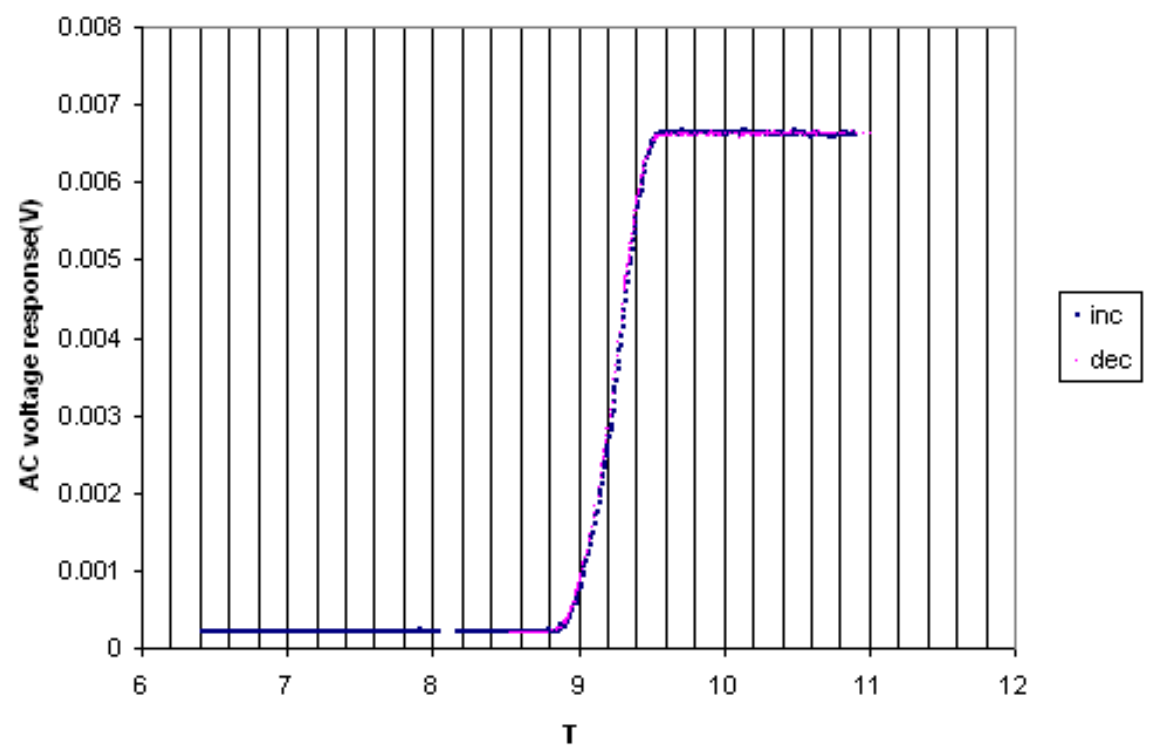

Fig. 5-6 Transition temperature for Niobium thin film on Copper by AC induction measurement 
Figure 5-6 is the transition temperature measured at Cornell University [52]. Since the pickup coil signal is processed by a non-phase locking amplifier, the low end detail gets buried in noise, thus the measurement shows a better transition width than the measurement the author has obtained.

Following tradition, the transition temperature is determined as the mid-point of $10 \%$ and $90 \%$ of the transition signal, and the transition width is the temperature difference between $10 \%$ and $90 \%$ of the transition signal. One has to note that the transition width for the inductive coil method is usually larger than that for the resistive method.

\section{5-2. RRR}

RRR is a short name for Residual Resistivity Ratio. It is defined as the ratio of metal's resistivity at $300 \mathrm{~K}$ over the resistivity at low temperature, which is considered the metal's residual resistivity. In the metal, the electrons get scattered by lattice vibrations, grain boundaries and impurities. When the temperature drops to a certain level, the metal shows the saturated electrical resistivity, which is mainly due to the electron scattering by impurities and grain boundaries. For metals like niobium, the grains are quite big, of micron size. The RRR of niobium is a good physics parameter to represent the purity, since the resistivity caused by grain boundaries is very small.

$$
R R R=\frac{\text { resistivity }(300 K)}{\text { residual_resistivity_at_low_temperature }(10 K, \text { or_lower_at_normal_state })}
$$

At low temperature, electrons are major carriers for heat. High RRR usually reflects the metal's heat conducting ability at low temperature, as is shown in figure 1-1 of chapter one.

It is different for small grain size thin films. The increased density of grain boundary contributes to the electron scattering, which yields a lower RRR for thin film material.

Experimentally, the RRR is measured by the four point resistive method as is shown in figure 5-7. For niobium thin film, the substrate has to be a non-conductor like a sapphire substrate. 


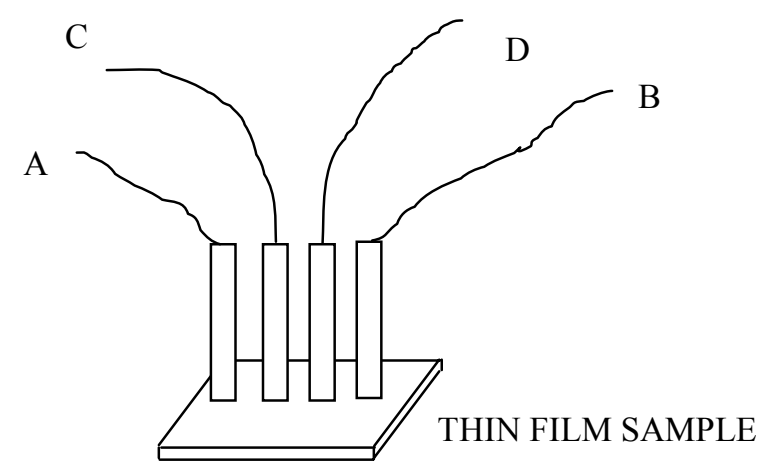

Fig. 5-7 Niobium thin film RRR measurement by the four point resistive method

Gold coated heads, A, B, C, D are spring loaded to provide close contact with the film. A constant current is fed into leads A and B, while a high sensitivity voltmeter reads the voltage across the leads $\mathrm{C}$ and $\mathrm{D}$. In practice, a switching current source is used to reduce the thermal EMF noise, and the voltage data is recorded for a wide range of temperatures. Figure 5-8 shows a typical RRR measurement curve on one niobium thin film sample. The RRR of the film was 27.

\section{RRR Measurement Curve}

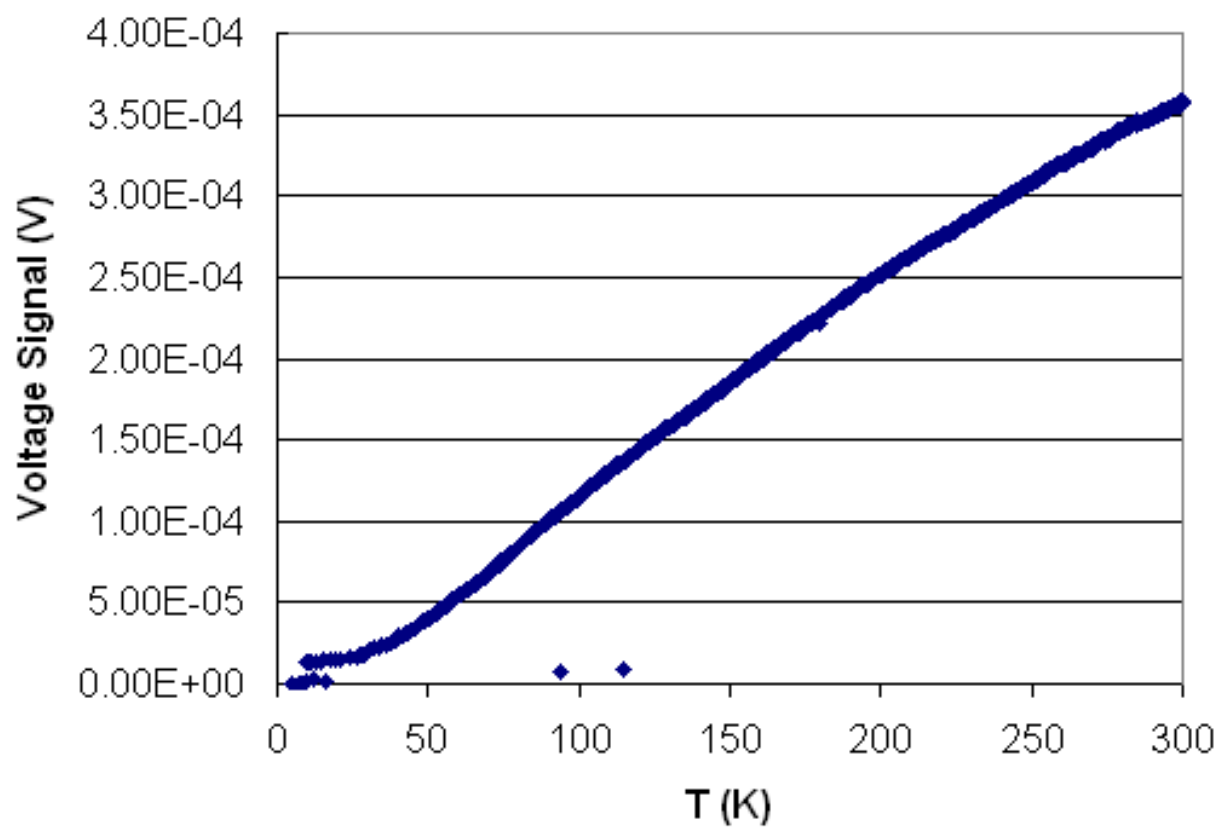

Fig. 5-8 A typical RRR curve measured on $\mathrm{Nb}$ thin film on sapphire 


\section{5-3. Critical magnetic field}

Niobium, as a type II superconductor, has lower and upper critical magnetic fields which characterize its superconducting properties in external magnetic fields. The upper critical magnetic field is one of the parameters affecting the surface resistance as we saw in chapter one. In early niobium thin film experiments, the upper critical magnetic field varies up to 1.5 Tesla [1]. In order to measure the upper critical magnetic field for a thin film sample, a uniform strong magnetic field is essential for good measurement. One superconducting coil has been made to fulfill such a need and is shown in figure 5-9. The Kapton tape covers each layer to smooth the winding of next layer. GE-varnish is coated on the final layer of wires to keep the winding from coming loose.

The magnet wire is made of NbTiN, 0.011-inch diameter. The later measurement shows the wire density at 3110.2 turns per meter for a single layer. With a current of 50 amperes,

$$
B=\mu_{0} n I=4 \pi \cdot 10^{-7} \cdot 3110.2 \cdot 50=0.19542 \text { Tesla. }
$$

A ten-layer winding yields about 1.9542 Tesla roughly. The final coil size is 3.25 -inch inner diameter and 9-inch in length. In table 5-2, the magnetic field is calibrated using a hall probe running through center axis of the coil. The center of the coil is located at $45.55 \mathrm{~cm}$ while the start and end $\mathrm{Z}$ positions of aluminum mandrel are at $32.55 \mathrm{~cm}$ and $62.55 \mathrm{~cm}$, respectively. Within four inches around the center of coil, the field is off from its maximum by $0.44 \%$.

To measure the upper critical magnetic field, the RRR measurement device shown in figure 57 is attached inside the coil mandrel and the film is aligned to be parallel to the magnetic field. By monitoring the resistivity change as a function of increasing coil current, the upper critical field can be obtained by scaling the magnetic field value according to the calibration data.

For thin film, the driven current also contributes to the magnetic field strength. During the experiment, driven currents ranging from $1 \mathrm{~mA}$ to $100 \mathrm{~mA}$ are tested. Most films hold the zero resistivity even at $100 \mathrm{~mA}$, with exception of one very thin sample which quenched at $65 \mathrm{~mA}$ driven current. Thus, $1 \mathrm{~mA}$ driven current is used through out the $\mathrm{B}_{\mathrm{c}}$ measurements. 


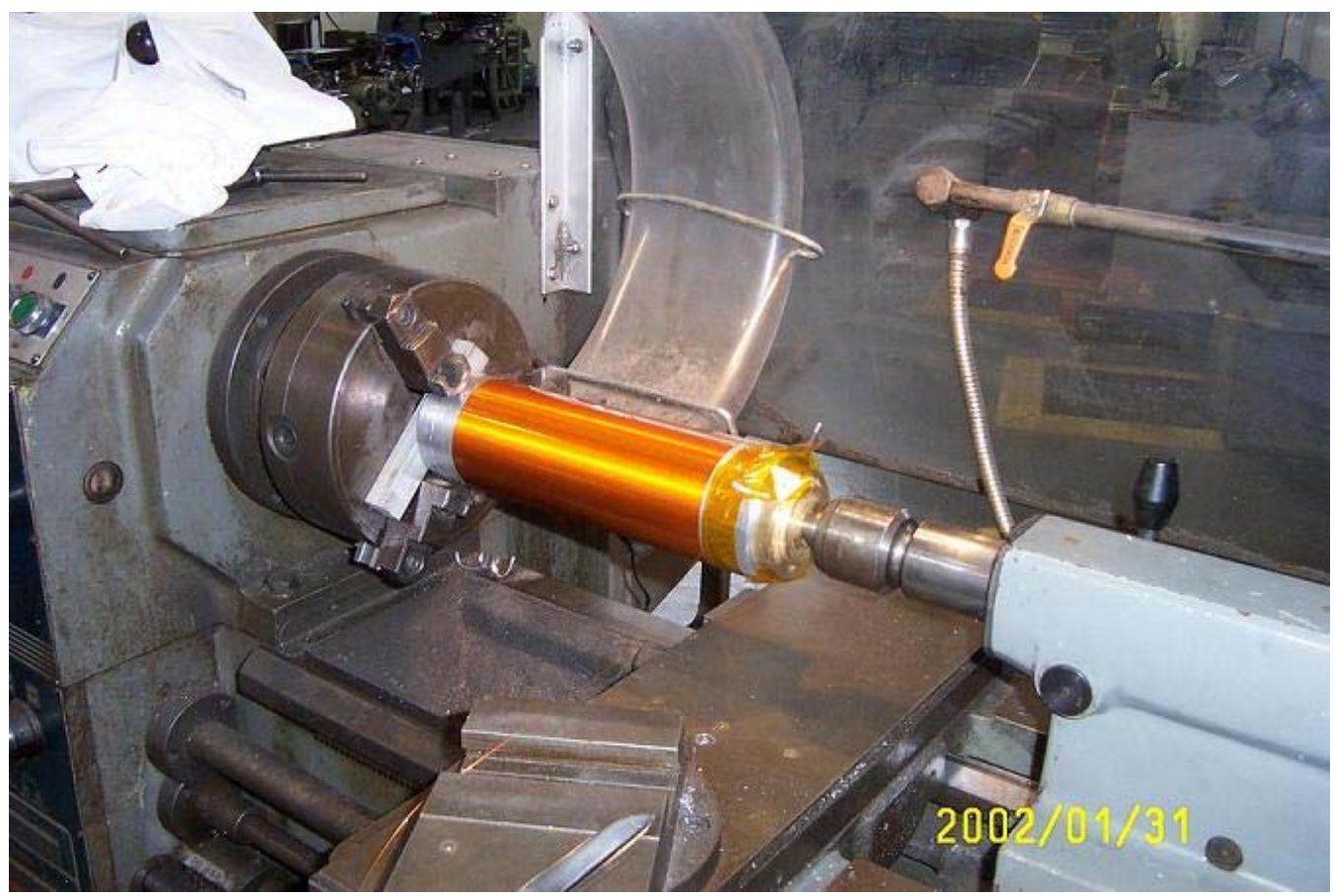

The coil is being wound on a lathe

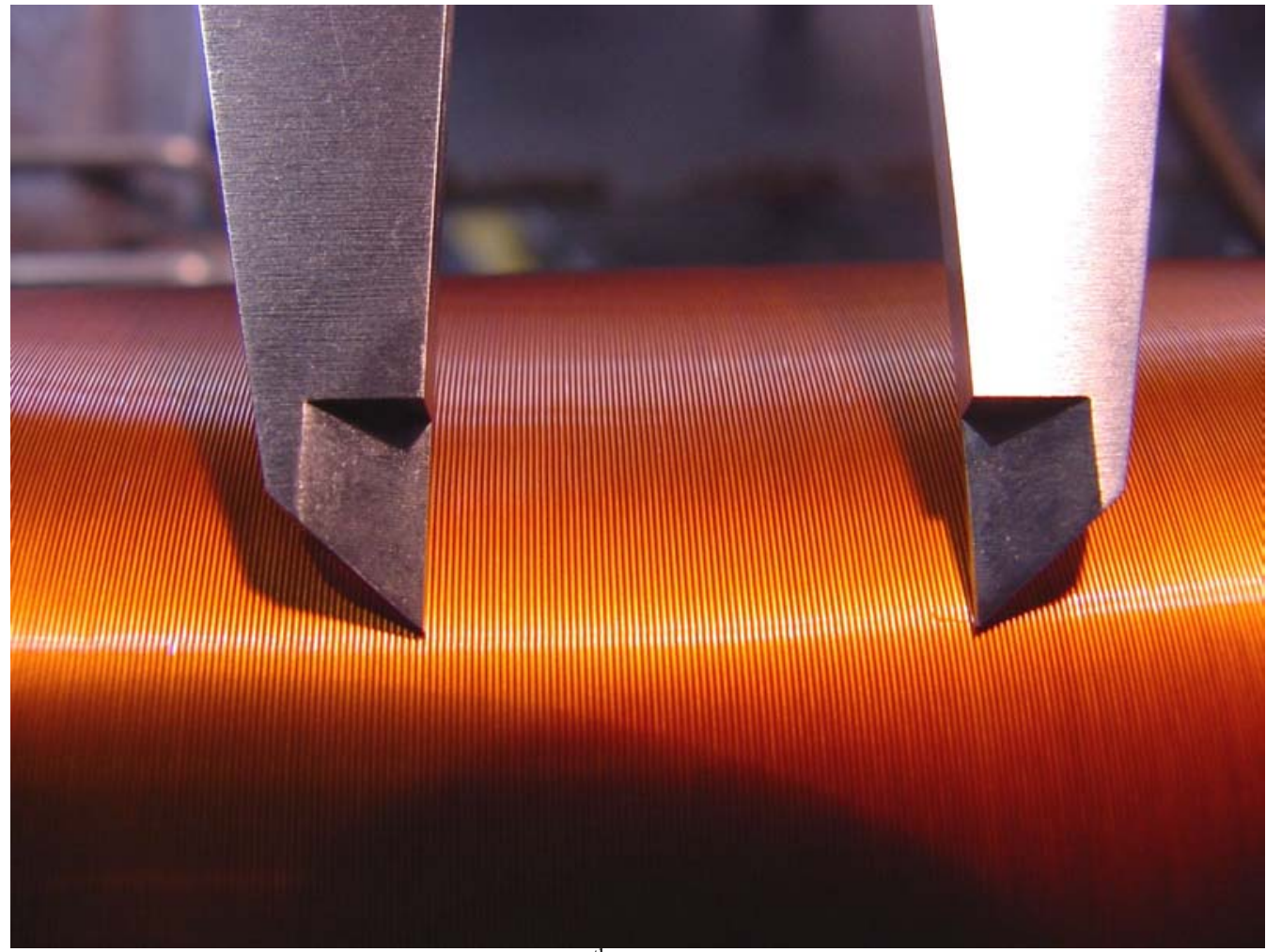

The close-up look for the $10^{\text {th }}$ layer, the caliper was at one inch.

Fig. 5-9 The making of superconducting coil for upper critical magnetic field measurement 
Table 5-2 The calibration data for magnetic coil with $100 \mathrm{~mA}$ current

\begin{tabular}{|l|l|}
\hline$Z(\mathrm{~cm})$ & $\mathrm{B}$ (Gauss) \\
\hline 45.5 & 29.51 \\
46 & 29.57 \\
46.499 & 29.6 \\
47 & 29.63 \\
47.5 & 29.64 \\
48 & 29.64 \\
48.5 & 29.6 \\
49 & 29.58 \\
49.501 & 29.55 \\
\hline
\end{tabular}

\section{Coil field for $100 \mathrm{~mA}$ Current}

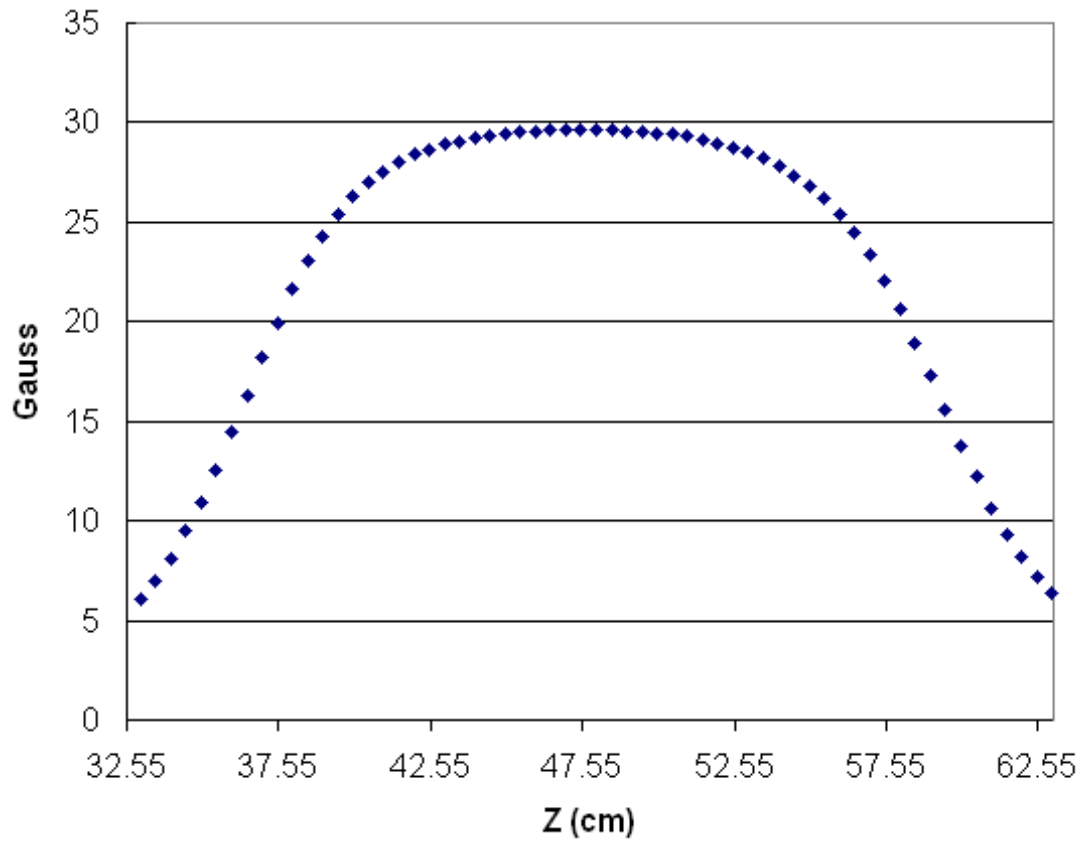

Fig. 5-10 The field contour of superconducting coil at center axis 
Coil field for $100 \mathrm{~mA}$ Current

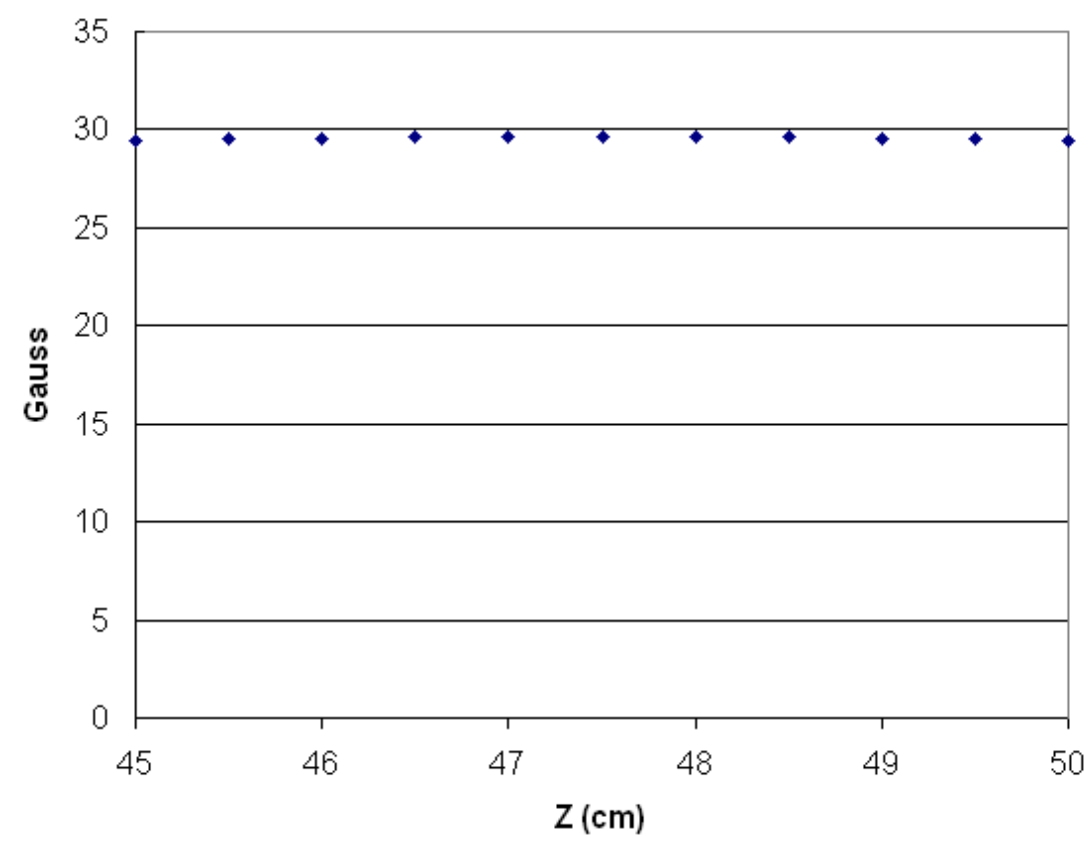

Fig. 5-11 The magnetic field of superconducting coil for center 5-inches

\section{5-4. Film thickness by different methods}

Film thickness can be obtained when the film is being made or after the deposition process. During the energetic deposition process, the thickness monitor should not be placed inside the plasma chamber for many reasons. Three different methods are used throughout the thin film characterization process. SEM and surface profilometer are two common methods. The electron microprobe analysis is also a good tool. When using SEM, the substrates are pre-cut at the backside before depositing the films. Mechanically splitting the substrate reveals a cross section of the film, which can be viewed under SEM to check the thickness and sometimes the film structure. One of such samples is shown in figure 5-12, the film thickness is determined to be 1.7 $\mu \mathrm{m}$. When the film thickness is at sub-micron level or even nanometer level, the microprobe analysis is better suited for the thickness measurement [53]. The SEM method is very accurate, but becomes difficult when the film thickness gets smaller. The surface profilometer measurement can be very quick, but has the risk of scratching a film. The microprobe analysis can be quick and causes no harm to a sample, but the accuracy is only around $5-10 \%$. 


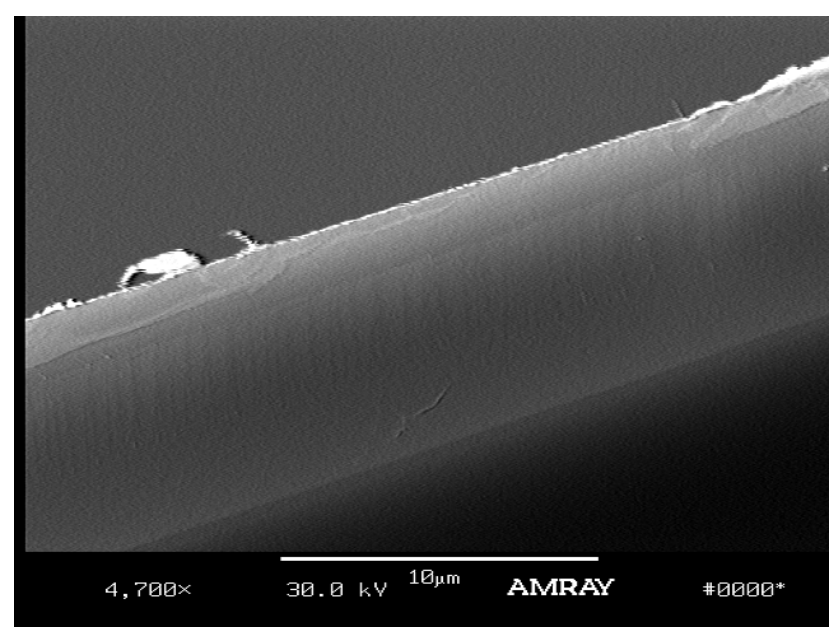

Fig. 5-12 Niobium thin film cross section on silicon. (SEM)

\section{5-5. Crystallite analysis by GADDS x-ray}

By using X-ray diffraction, one can obtain crystallite size and the crystal orientation disorder parameter. Further analysis of X-ray data on thin films can also give us the crystal "lattice constant" which can be used to compare with solid niobium to understand the crystal lattice deformation caused by Hydrogen "contamination" [54], also the residual stress of the film.

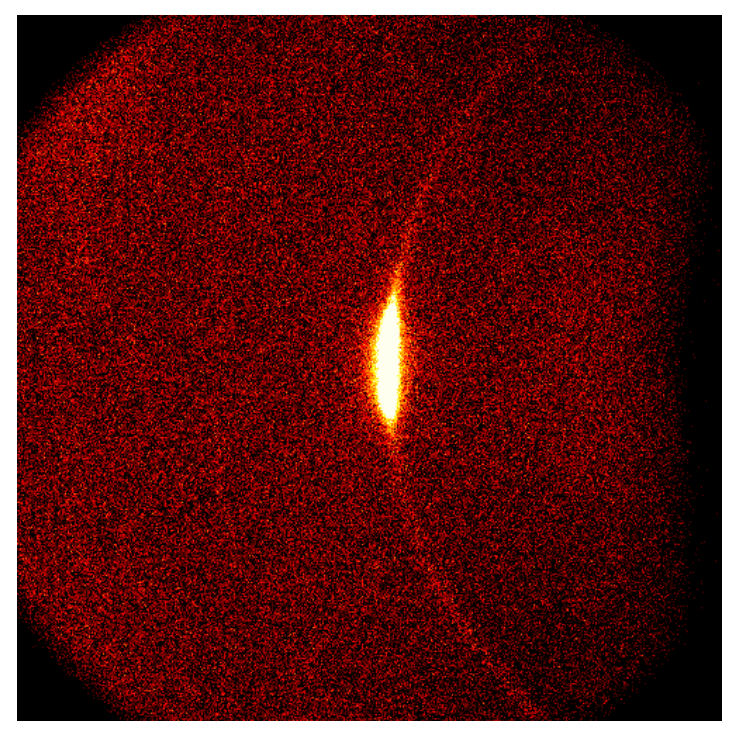

Fig. 5-13 The GADDS X-ray result shows a well-aligned crystal structure for $\mathrm{Nb}$ thin film on sapphire substrate. 
Although the SIMS would provide much more information on depth profile for Hydrogen and other element like oxygen, the X-ray measurement gives us instant and rich information on the sample.

Figure 5-13 shows X-ray diffraction data for one thin film. The crystal mis-orientation is about 5 degrees. The mis-orientation parameter is the FWHM value of the diffraction signal. An ideally aligned crystal would give a perfect round spot. The crystal size and lattice parameter are not measured at this time.

\section{5-6. Surface roughness and grain size by AFM}

The now very popular Atomic Force Microscope is easily accessible, and it is a powerful tool to look at detailed surface structure. From AFM data, we can instantly get the degree of surface roughness and surface grain size and structure. As an example, figure 5-14 shows a very flat niobium surface with grain size about 80-100 nm.

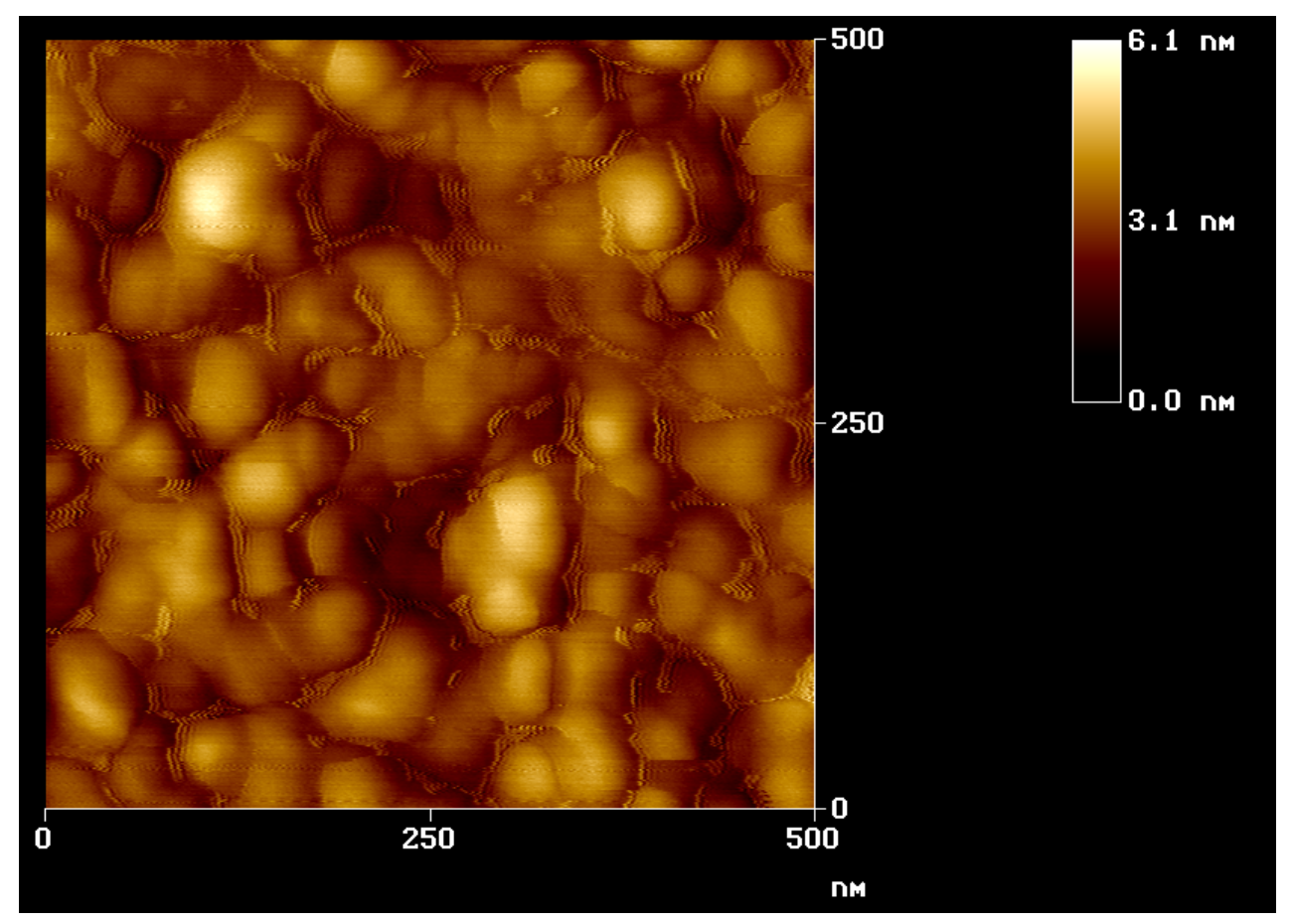

Fig. 5-14 The AFM picture shows a flat, densely packed, niobium thin film on a sapphire substrate with grains of around $80 \mathrm{~nm}$ sizes 


\section{5-7. The surface quality check by Optical microscope and SEM}

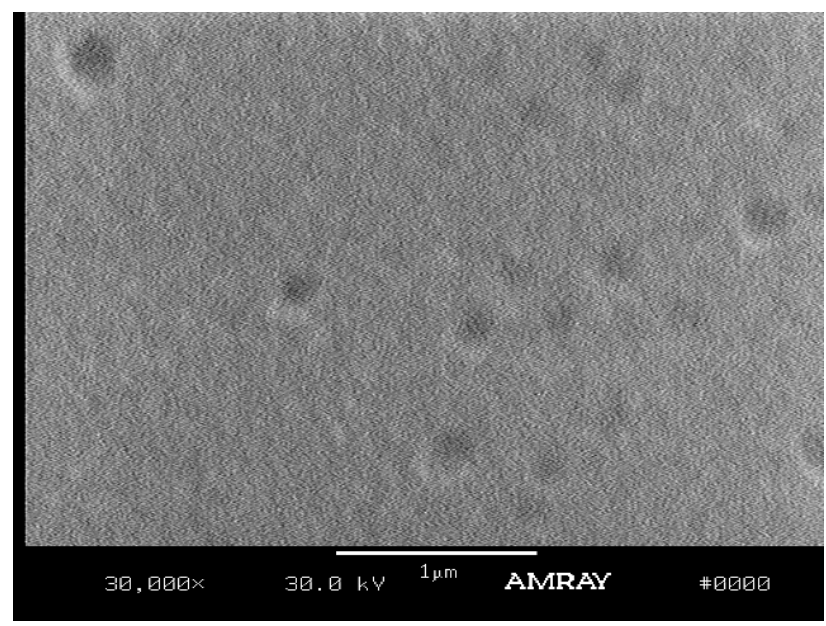

Fig. 5-15 Niobium thin film on copper (SEM)

An optical microscope is utilized to pre-check the surface quality for major defects. Once that is done, SEM is used to do further surface quality checks for small-scale defects and the material composite of the defects can be checked if necessary. Figure 5-15 is a niobium thin film on a copper surface. The small pits have the size from $100 \mathrm{~nm}$ to $200 \mathrm{~nm}$, and are inherited from the copper substrate. 


\section{Chapter 6. Niobium Thin Film Deposition and the Properties at Different Deposition Energies}

\section{6-1. Overview of Niobium Thin Film Deposition}

During niobium thin film deposition, the deposition energy is the main parameter to investigate. All other deposition parameters are roughly kept the same. The vacuum is around $1.0 \times 10^{-7}$ torr before the deposition and around $1.0 \times 10^{-6}$ torr during the deposition. The residual gas content is dominated by hydrogen. The RF power is kept roughly the same at 250 watts input power. The RF power will be the major factor affecting the plasma, thus the voltage potential of plasma is fixed at 64 volts as it is measured by the retarding field energy analyzer. The deposition rate is dependent on the deposition energy, beam power and RF power, and it is the only parameter that is not well controlled. The deposition rate ranges from $2.5 \AA / \mathrm{s}$ to $5.2 \AA / \mathrm{s}$ and is limited by substrate charging during some sapphire based sample preparations. The niobium thin films are made for several sets of substrate bias voltages. Many films are made on sapphire substrates and silicon substrates. Some of the films are made on copper substrates.

The sapphire substrate films are the most extensively explored niobium thin films. The silicon films are made at the same time as the sapphire substrate films. The silicon substrate films are used for thickness monitoring and sometimes for the $T_{c}$ measurement. The sapphire substrate films are extensively characterized through $T_{c} / R R R / B_{c}, X$-ray, AFM and SEM. The copper substrate films are checked for $\mathrm{T}_{\mathrm{c}}$, SEM and DC field emission.

The silicon substrate is cleaned by methanol rinsing and nitrogen blow dry. The sapphire substrate is cleaned very thoroughly by a 5-minute trichloroethylene warm bath and a 20-30 second 5\% HF rinsing, then methanol rinsing. The copper substrate is electropolished by a phosphoric acid and butanol composition in 50-50 ratio. A 200-micron layer is removed during 3-4 hour electropolishing. The thin film samples are categorized as VV-SS-NN, where VV denotes the substrate voltage, SS denotes the substrate and NN denotes the individual films. The films are selected depending on their acceptable thickness. 


\section{6-2. The vacuum condition}

The vacuum chamber has excellent base pressure, as low as mid $10^{-9}$ Torr as mentioned in chapter three. After many deposition runs, the base pressure degraded to mid $10^{-8}$ Torr. That is achieved through 72 hours pump down with partial chamber bake. The partial bake is done though heating the different chamber wall sections, mostly the chamber bottom. Because of the niobium surface pumping effect, the usual pumping time is 24 hours for each film deposition.

During the ECR plasma operation, most of system residual gas comes from E-beam gun operation. The melting point of niobium is about $2468{ }^{\circ} \mathrm{C}$. This is not surprising considering the E-beam gun consumes about $10 \mathrm{~kW}$ power to evaporate the niobium target. Since the E-beam gun itself is effectively cooled by chilled water, the hot source is the niobium target inside the Ebeam gun. The niobium target is made in high purity (RRR as 250). Since the E-beam gun consumes a lot of niobium material, the target is replenished for every film deposition. For convenience, niobium pellets are used. All the niobium pellets are carefully cleaned by acid etching and methanol rinsing. To further reduce the outgasing, niobium pellets are annealed at $800^{\circ} \mathrm{C}$ inside a $10^{-5}$ torr vacuum furnace for 10 straight hours. For every film deposition, the niobium targets will undergo a two-hour target conditioning with the E-beam gun gradually increasing the beam power.

Since niobium is an excellent getter for vacuum residual gases, the E-beam evaporation before the film deposition is a real benefit in order to obtain better vacuum. For a $50{ }^{\circ} \mathrm{C}$ niobium surface, a hydrogen initial sticking coefficient of 0.02 [55] can be used to estimate the pumping speed of niobium surface. Pumping speed has the formula:

$$
S=1000 A \frac{v}{4} S_{i}
$$

where $\mathrm{S}$ is pumping speed in liter per second, $v$ is the gas velocity in $\mathrm{m} / \mathrm{s}, \mathrm{A}$ is the surface area in units of $\mathrm{m}^{2}$ and $s_{i}$ is the initial sticking coefficient. For $1 \mathrm{~cm}^{2}$ niobium surface area, the surface pumping speed for hydrogen is $0.875 \mathrm{l} / \mathrm{s}$, if hydrogen has velocity of $1,600 \mathrm{~m} / \mathrm{s}$. 
As the calculation in section 3-4 in chapter three shows, the evaporation rate of $100 \AA / \mathrm{s}$ of niobium flux represents the niobium number density $n_{n b}$ of $8 \times 10^{17} / \mathrm{m}^{3}$. The hydrogen number density $n_{\mathrm{H} 2}$ can be computed through:

$$
n_{H_{2}}=\frac{3 P}{m v^{2}},
$$

where $\mathrm{P}$ is the partial pressure, $\mathrm{m}$ is the hydrogen mass and $\mathrm{v}$ is the hydrogen velocity. For hydrogen partial pressure of $1.0 \times 10^{-6}$ Torr, the hydrogen number density is $4.7 \times 10^{16} / \mathrm{m}^{3}$. The number ratio of hydrogen and niobium is

$$
\frac{2 n_{H_{2}} v_{H} s_{i} \frac{1}{6}}{n_{N b} v_{N b}}=0.001,
$$

where $v_{H}$ is hydrogen velocity of $1,600 \mathrm{~m} / \mathrm{s}, v_{N b}$ is niobium velocity of $630 \mathrm{~m} / \mathrm{s}$. The factor of $1 / 6$ is geometric factor for a flat surface where hydrogen hits. At room temperature, the solubility limit for hydrogen in niobium is 7\% [56]. The hydrogen ratio of 0.001 is well within the limit. The fast rate of niobium impinging on the surface can also cause the enhanced sticking coefficient because the niobium intends to bury or hit back the hydrogen escaping from surface. Inside the circular waveguide, fast rate niobium hits a surface area around $20 \mathrm{~cm}^{2}$, which represents $16 \mathrm{l} / \mathrm{s}$ local pumping speed just for hydrogen. That helps to overcome the pumping impedance introduced by two small openings. One has to note the situation can be complicated by the circular waveguide heating and the complex geometry of the vacuum system.

The typical vacuum condition change during film deposition is shown in Appendix A. Initially, the residual gas contains mostly hydrogen, nitrogen, carbon dioxide and dominating water vapor. After the E-beam gun starts to warm up, extra water vapor is released from the niobium targets, e-beam gun body and nearby surface areas. When the E-beam gun increases its power, more water vapor is released, and hydrogen starts to become more dominant. In about two hours of E-beam gun running, all the residual gases' partial pressure starts to decrease. A small increase of E-beam gun power will start to evaporate a small amount of niobium vapor. The newly evaporated niobium deposits onto the surfaces inside the vacuum chamber, and further decreases the residual gas content as surface pumping comes into play. By the time the deposition starts, residual gases are dominated by hydrogen. As the deposition continues, the E- 
beam gun power is kept at a high level to evaporate large amount of niobium vapor. About one fourth of the niobium vapor travels into the plasma reactor, the rest of niobium deposits onto the surfaces inside the vacuum chamber and also inside the plasma reactor. The speedy $100 \AA / \mathrm{s}$ newly deposited niobium surfaces act as a huge absorption pump to reduce residual gas pressure very effectively. By the time deposition stops and the E-beam gun power level is reduced, the system vacuum reaches lowest pressure. The time needed to reach the base pressure is days instead of weeks.

\section{6-3. The deposition parameters and film properties}

\section{6-3-1. Deposition Rate}

Deposition time for each film is recorded. Deposition rate is then obtained after thickness measurement through surface profilometer or electron microprobe analysis. The rate is not well controlled but ranges typically from 3 to $4 \AA$ /s as seen in table 6-1.

\section{Table 6-1 Deposition rate for the films being tested}

\begin{tabular}{|l|r|}
\hline Film & Rate $(\AA / s)$ \\
\hline $02-S A-1$ & 3.9 \\
$25-S A-3$ & 3.9 \\
$51-S A-1$ & 2.92 \\
$51-S A-3$ & 5.2 \\
72-SA-5 & 3.8 \\
100-SA-1 & $\sim 2.4$ \\
\hline
\end{tabular}

It is observed that substrate charging is present. One deposition test on silicon substrate with and without gold coating yields film thickness of $700 \AA$ and $220 \AA$, respectively. All the sapphire substrates are gold coated along the edge. However, the same batch of films still show large variations of thickness from time to time; suggesting the substrate holder is in poor contact with the substrate gold tab. This could explain the relatively low and variable deposition rates. 


\section{$\mathbf{T}_{\mathrm{c}}$ vs. Bias Voltage}

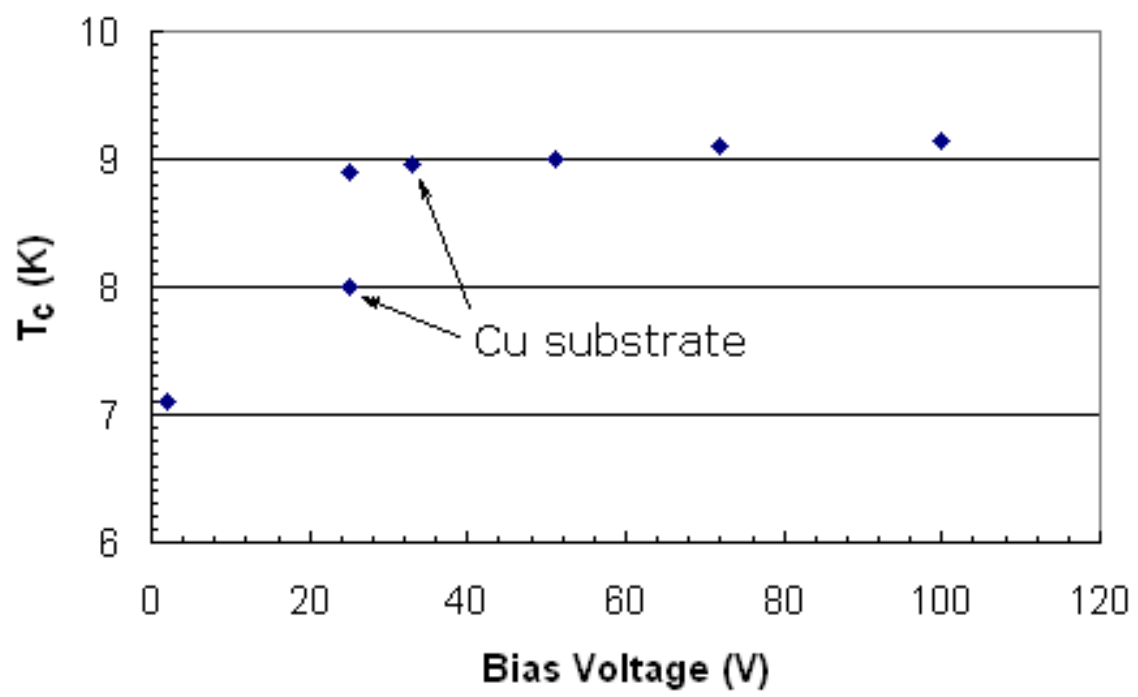

Fig. 6-1 Bias voltage effect on transition temperature measured by inductive method

\section{$\Delta \mathbf{T}_{\mathrm{c}}$ vs. Bias Voltage}

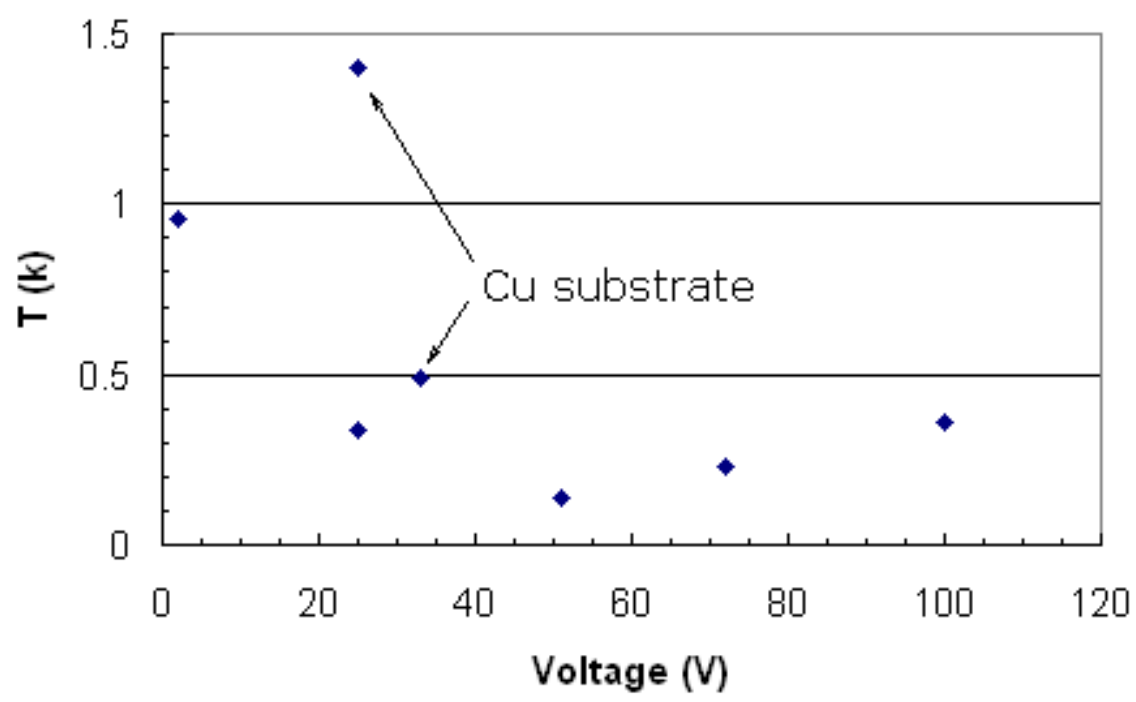

Fig. 6-2 Bias voltage effect on transition width 


\section{6-3-2. Transition Temperature}

For sapphire substrates, the thermal expansion coefficient is $5.6 \times 10^{-6} / \mathrm{K}$, while for niobium it is about $4 \times 10^{-6} / \mathrm{K}$. After cooling down to the liquid helium temperature, the niobium films on sapphire will experience compressive stress. It is interesting to notice that all the films have a slightly lower transition temperature than solid niobium. Whether it is caused by the film compressive stress can be determined by the future measurements of the lattice spacing. From figure 6-1, it is interesting to notice that high deposition energy shifts the transition temperature close to that of solid niobium. The first data in the figure shows a much lowered transition temperature. The possible cause is that there is methane content during the deposition. A vacuum part had trapped excessive methanol during chemical cleaning. It is also worth noting that the substrate is collecting the niobium just like in the sputtering deposition. The niobium ions come to the substrate solely by plasma expansion, possibly not at the preferred 90-degree incident deposition angle.

As stated in chapter five, if the transition width reflects the overall film quality, then the results in figure 6-2 suggest that a substrate voltage around $-51 \mathrm{~V}$ is preferable, or a niobium kinetic energy around $115 \mathrm{eV}$ is the preferred deposition energy.

\section{6-3-3. RRR, $B_{c}$ and Film Thickness}

Due to the limitation of the cryogenic system at Jefferson Lab, the upper critical magnetic field is measured at a fixed temperature. The maximum current allowed to the cryogenic dewar is also limited at $50 \mathrm{~A}$. The result of the $\mathrm{B}_{\mathrm{c}}$ measurement is incomplete at this stage.

At least three more factors will contribute to the resistivity at low temperature. They are impurity and crystal defects, electron scattering caused by dense grain boundaries, and electron surface scattering $[57,58]$. The surface scattering decreases the effective electron mean free path in very thin films. A coarse estimation [58] shows that the resistivity could double for films with thickness comparable to the electron mean free path in the solid. Since the thinner niobium film has roughly the same grain size as the thickness, it is believed that surface scattering could double the electrical resistivity at most. The small grains in those thinner films are likely 
responsible for the low RRR.

Table 6-2 RRR and upper critical field for different films

\begin{tabular}{|c|c|c|c|}
\hline Film & RRR & $\begin{array}{c}\text { Thickness } \\
(\mathrm{nm})\end{array}$ & $\mathrm{B}_{\mathrm{c} 2}(4.2 \mathrm{~K})$ \\
\hline 02-SA-1 & 2.31 & 68.2 & $>1.5 \mathrm{~T}$ \\
25-SA-3 & 6.8 & 126 & $>1.5 \mathrm{~T}$ \\
51-SA-1 & 2 & 74.1 & $>1.5 \mathrm{~T}$ \\
51-SA-3 & 50.2 & 235 & $0.96 \mathrm{~T}$ \\
72-SA-5 & 26.7 & 181 & $1.28 \mathrm{~T}$ \\
100-SA-1 & 4 & 79 & \\
\hline
\end{tabular}

\section{6-3-4. GADDS X-Ray Diffraction}

The films are also investigated by X-ray diffraction analysis. Table 6-3 shows the crystal alignment for different films. The 51 film shows extremely well aligned crystal orientation. The X-ray picture shown in figure 6-3 is almost identical to that of epitaxial niobium thin film.

Table 6-3 Crystal alignment by X-ray diffraction

\begin{tabular}{|l|l|}
\hline Film & X-ray \\
\hline $02-S A-1$ & $\sim 10-15$ \\
25-SA-3 & $\sim 3-4$ \\
$51-S A-1$ & $<1$ \\
$51-S A-3$ & \\
72-SA-5 & $\sim 5-6$ \\
100-SA-1 & $\sim 5$ \\
\hline
\end{tabular}

\section{6-3-5. AFM surface structure}

The films listed in the table are investigated by AFM. The Pictures are shown in figure 6-4.

The 02-SA film and 100-SA film have small round grains, while the 25-SA film shows elongated grains. For the 51-SA film and 72-SA film, the grains become extremely long, as is shown in figure 6-5. The difference between the long grained film and round grained film may be caused by the film thickness. Further TEM studies should reveal more about the grain structures. 


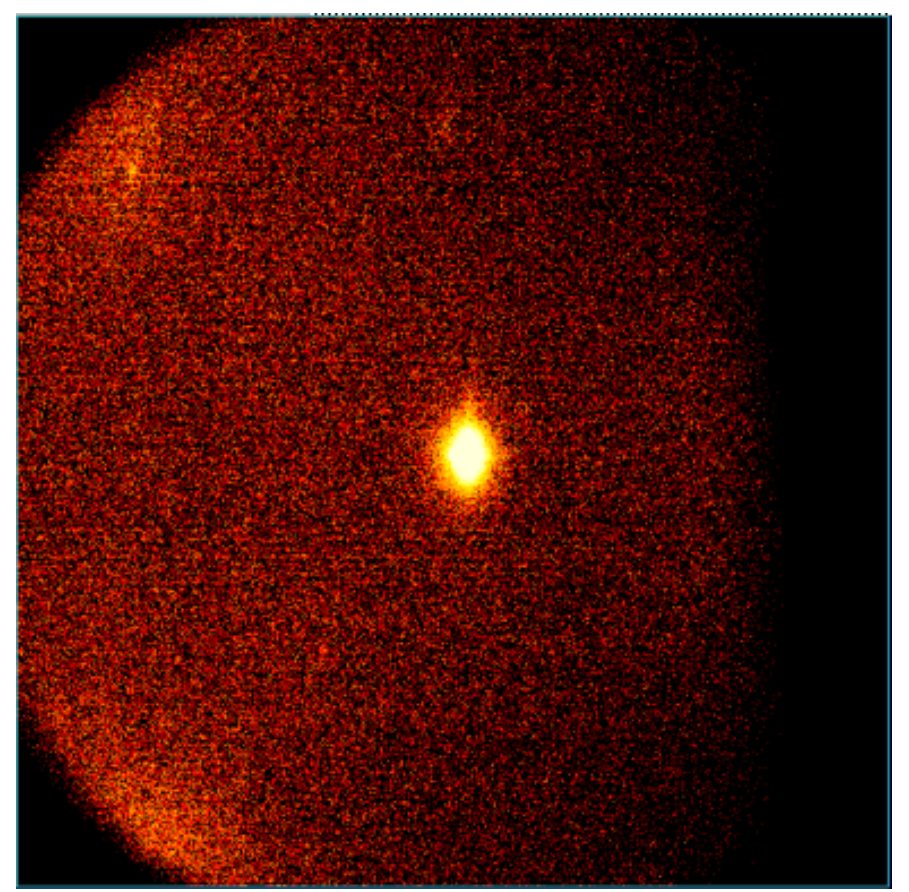

Fig. 6-3 X-ray diffraction image of 51 film 


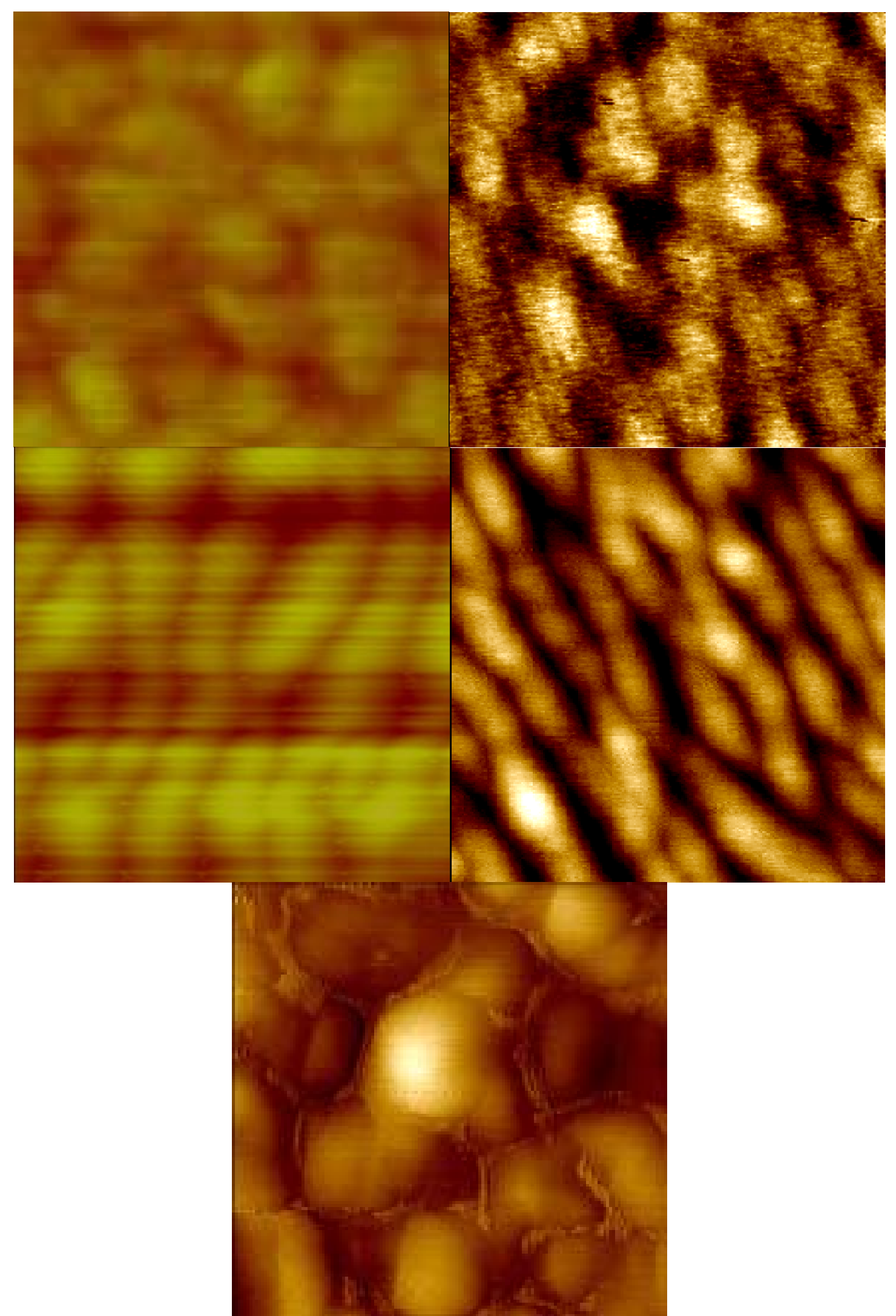

Fig. 6-4 From left to right, and top to down, the AFM images are for $02 \mathrm{~V}, 25 \mathrm{~V}, 51 \mathrm{~V}, 72 \mathrm{~V}, 100 \mathrm{~V}$ films, image sizes at 200 by $200 \mathrm{~nm}$ 


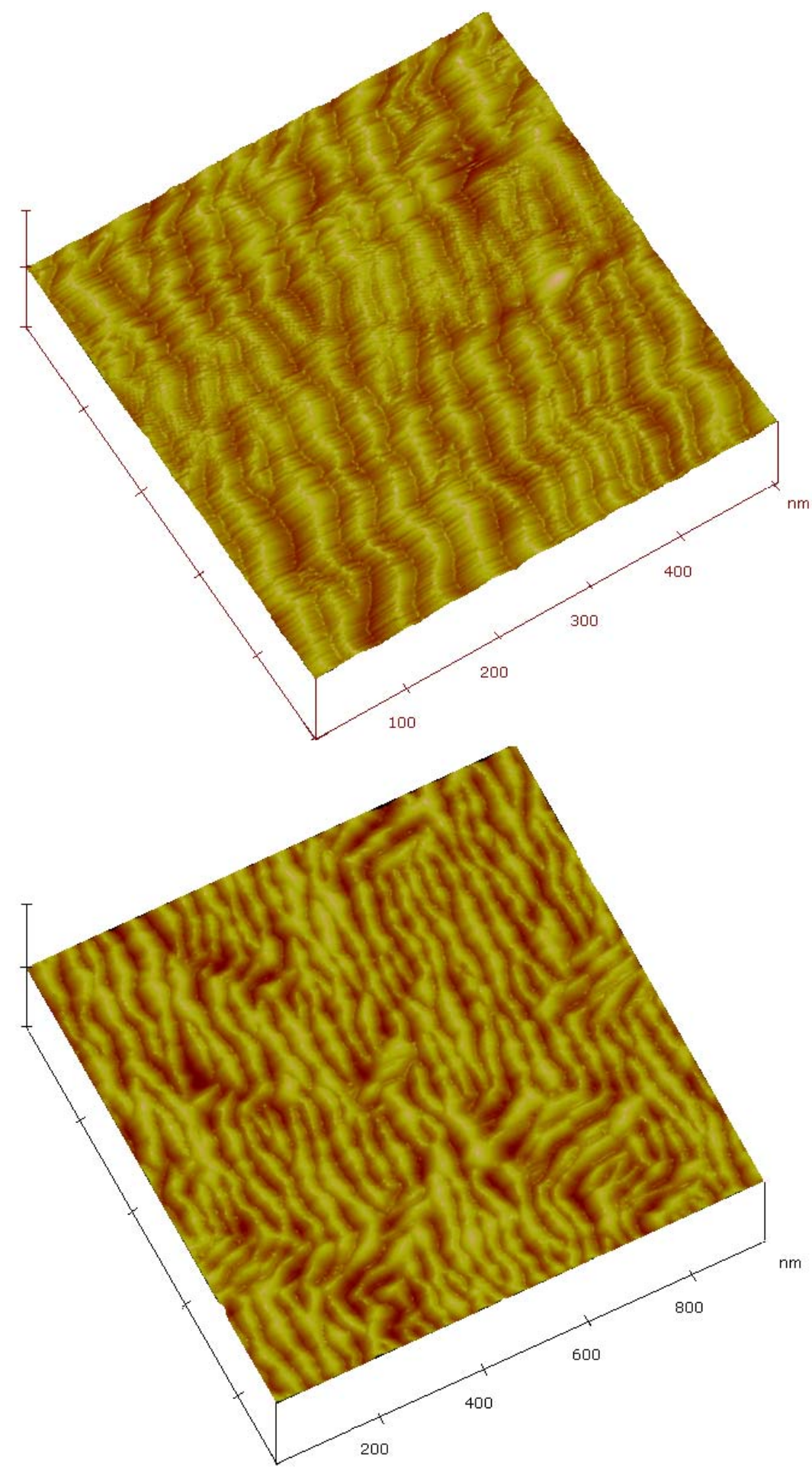

Fig. 6-5 Long continuous grains of 51V film 


\section{6-3-6. Field Emission Test of Copper based Sample}

DC field emission studies are conducted on solid niobium samples [20]. It is found that the DC field emission is a perfect tool to identify the various kinds of defects for niobium thin film. One niobium film deposited on a copper substrate at -25 bias voltage was scanned for field emission at $140 \mathrm{MV} / \mathrm{m}$.

The $\mathrm{Cu}$ substrate was electropolished to remove $200 \mu \mathrm{m}$ before the deposition. The copper substrate shows numerous small pits after the polishing process.

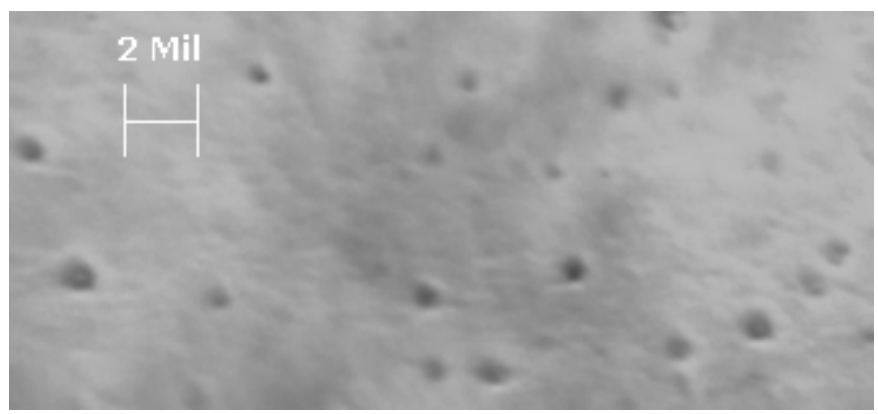

Fig. 6-6 Electropolished Copper surface under optical microscope

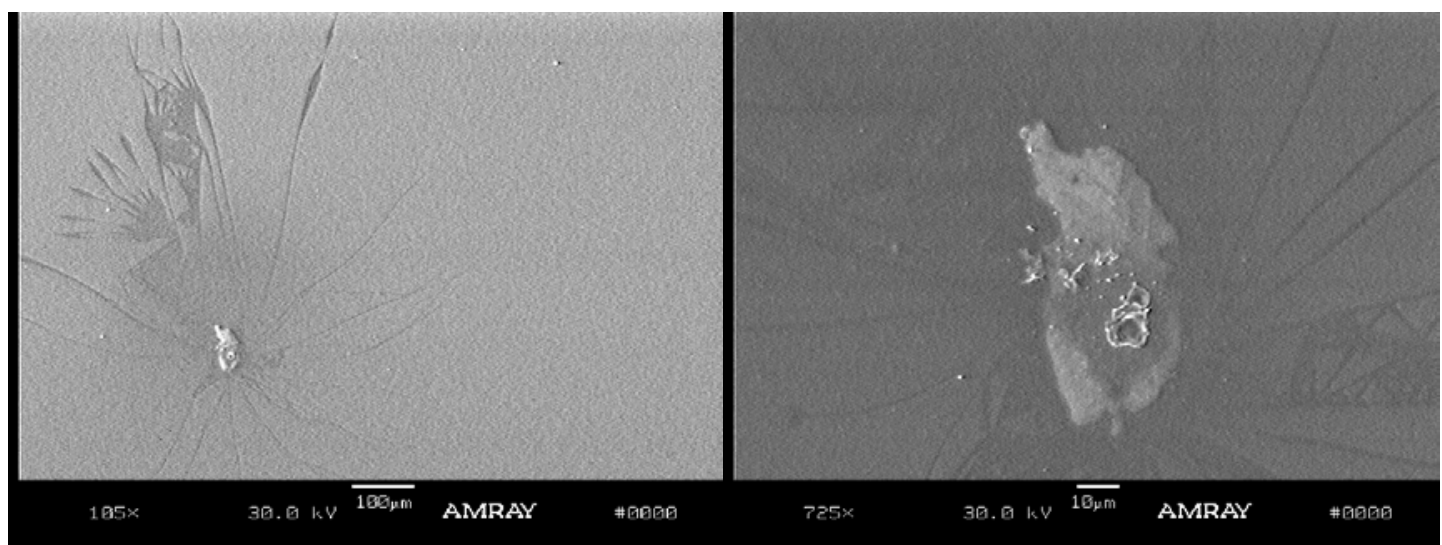

Fig. 6-7 One emitter at different magnifications (contains $\mathrm{Cu}, \mathrm{Fe}, \mathrm{Ni}, \mathrm{Cr}$ )

(Courtesy of T. Wang)

After the film was deposited, the sample was transferred in clean dessicator to the field emission chamber. A 2- $\mathrm{cm}^{2}$ sample surface area was scanned. Twenty sites had emission fields between 30 and $140 \mathrm{MV} / \mathrm{m}$. There were also 20 arcs. It was confirmed that dust particles and surface pits inherited from copper substrate caused these arcs. Later composition studies by EDX 
in SEM revealed that many emitting sites contain $\mathrm{Cu}, \mathrm{Fe}, \mathrm{Ni}, \mathrm{Cr}$, likely caused by the dust particles introduced during sample transfer. One such emitter site is shown in figure 6-7.

In some locations where neither dust particles nor surface pits are present, a surface area of $1 \mathrm{~mm}^{2}$ was scanned. No emission or arcing up to $140 \mathrm{MV} / \mathrm{m}$ was found; in other words, no evidence of intrinsic emission was observed from the film.

\section{6-4. Discussion}

It is understandable that the surface resistance together with maximum magnetic field and maximum electric field would be the decisive parameters when judging a film's quality for cavity production purposes. The other parameters can also reflect the quality of thin films. The superconductor transition under an inductive AC field is generally believed to reflect the overall film quality for relative large samples. RRR usually represents the purity level in solid niobium. For film, RRR can reflect overall quality in combination with purity level, grain boundary quality and crystal defects level inside a grain. Further studies from SIMS analysis and TEM analysis should be able to determine all three factors mentioned above. While the film thickness may play some role in the film structure, it is necessary to conduct more film depositions to verify the effect. As to the X-ray diffraction analysis, it is believed that some columnar films may also exhibit well-aligned crystal orientation even though their superconducting properties are poor. One possible advantage of thin films with a well-aligned crystal structure is that the electron mean free path may be greater.

\section{6-5. Conclusions}

Niobium ion creation by ECR plasma is successful. The controllable deposition energy is achieved through changing substrate bias voltage. The surface morphology can be changed by the deposition energy together with other deposition parameters. Based on results from the current collection of films, a relatively high deposition energy around $115 \mathrm{eV}$ is preferable. Most of the films have consistent sharp superconducting transitions around $9 \mathrm{~K}$. This suggests that energetic deposition in vacuum is generally better than other traditional coating processes (as shown in chapter 2). Field emission tests show no serious field limitations if the substrate is well polished and the surface is free of dust. 


\section{Appendix A. RGA Monitoring during One Deposition Run}

The RGA reading is not calibrated. The RGA total pressure is about half of the ion gauge pressure reading.

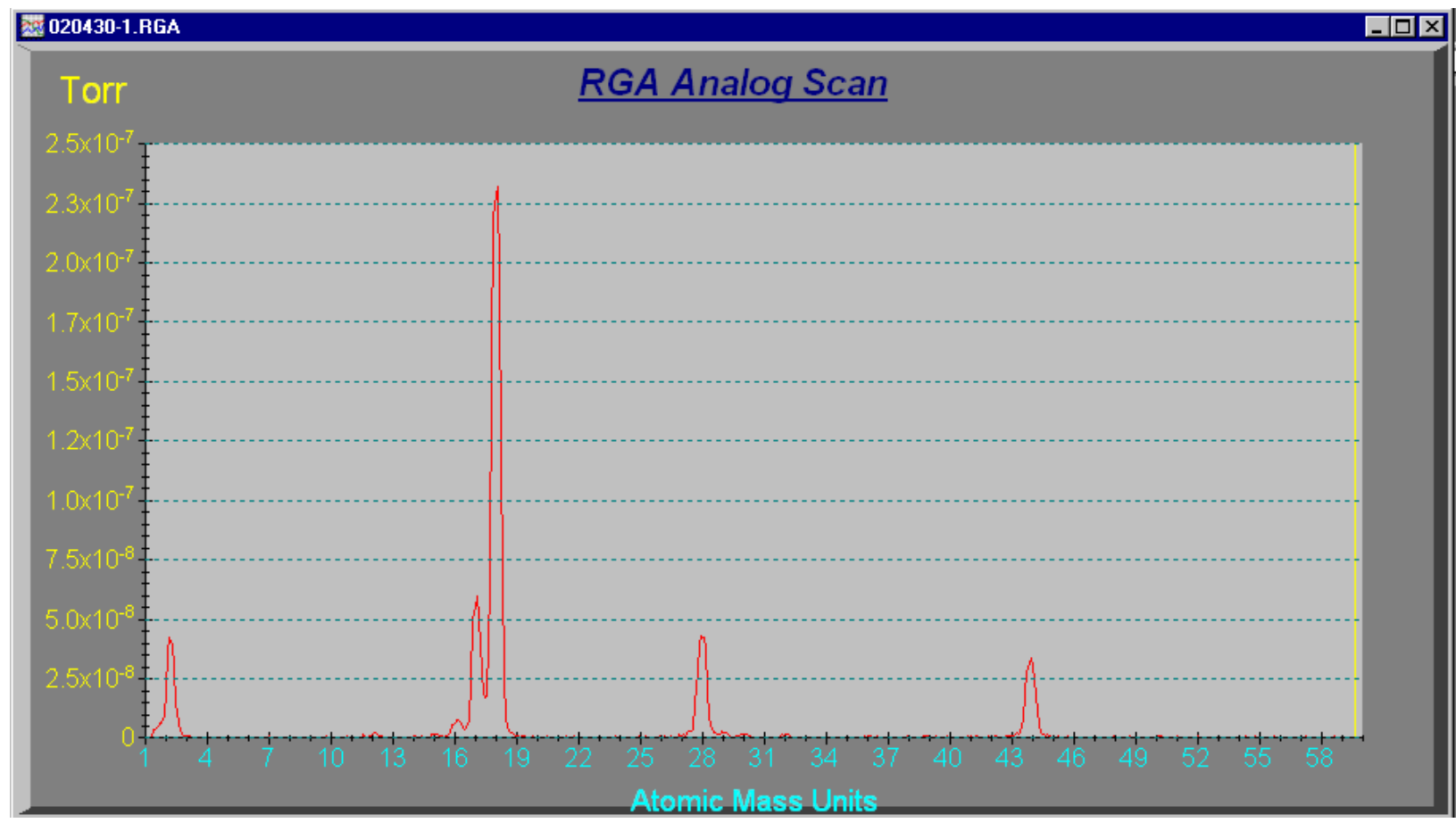

Initial Vacuum condition

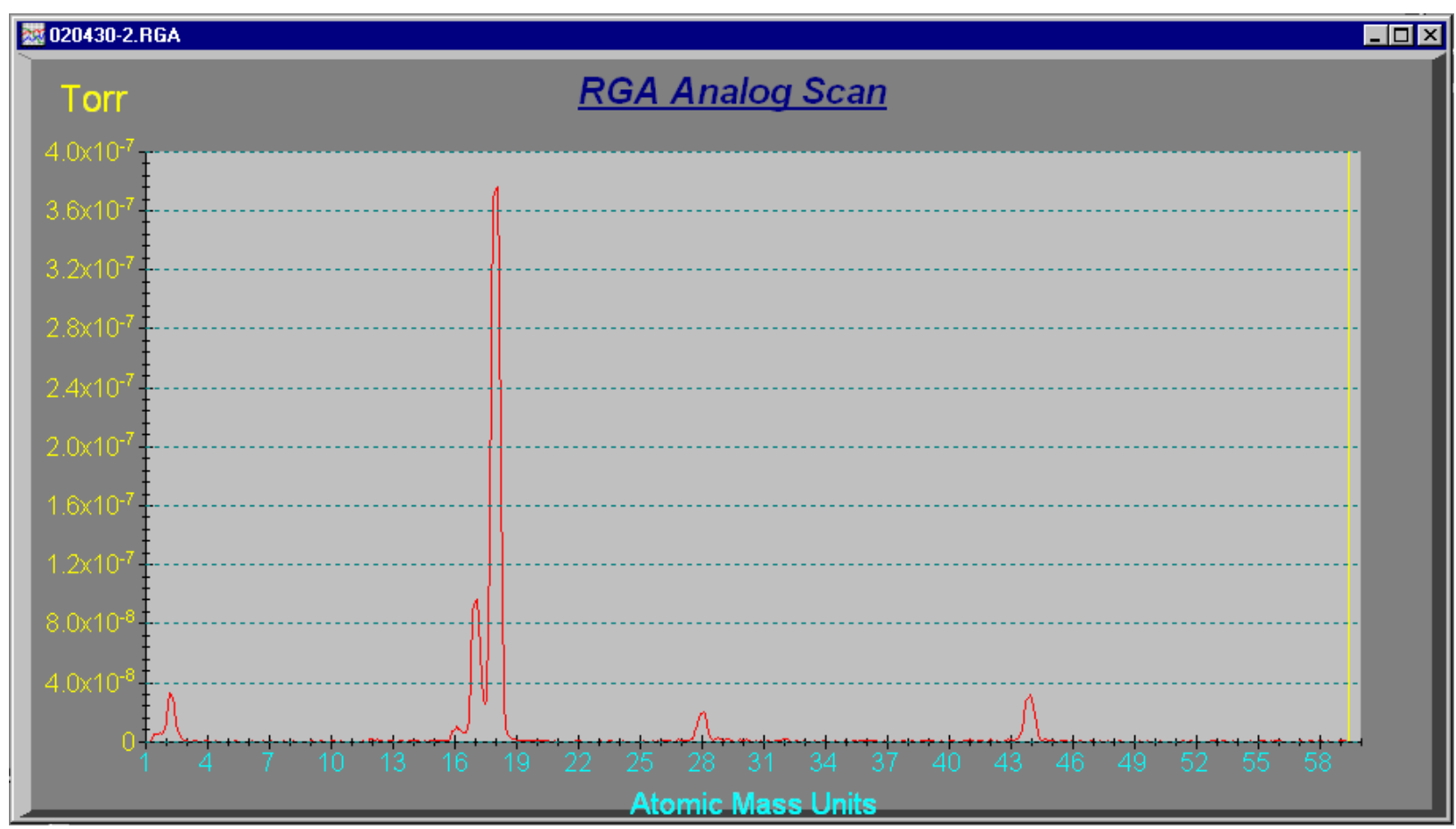

E-beam gun starts to warm up 


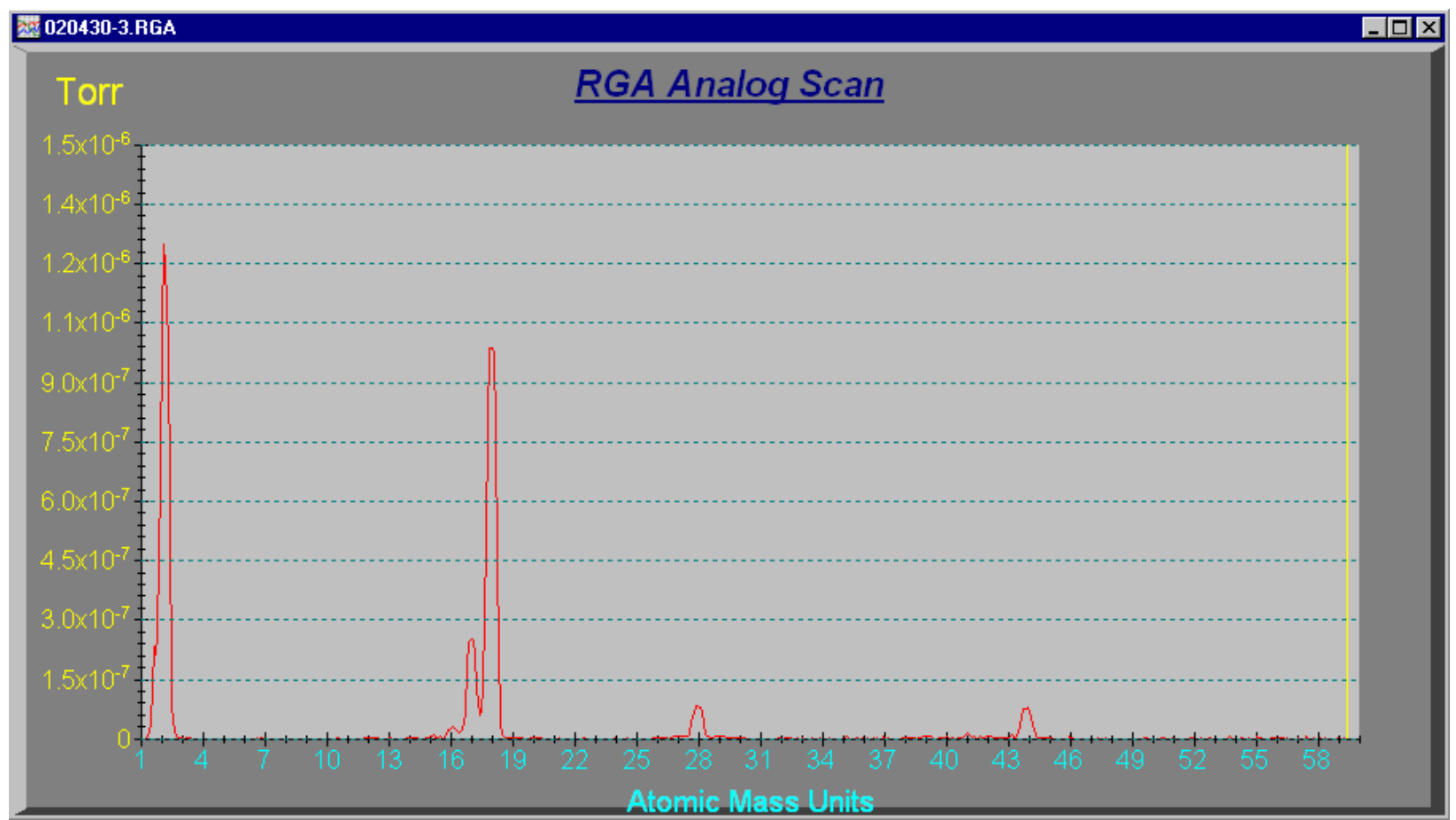

E-beam gun power increases

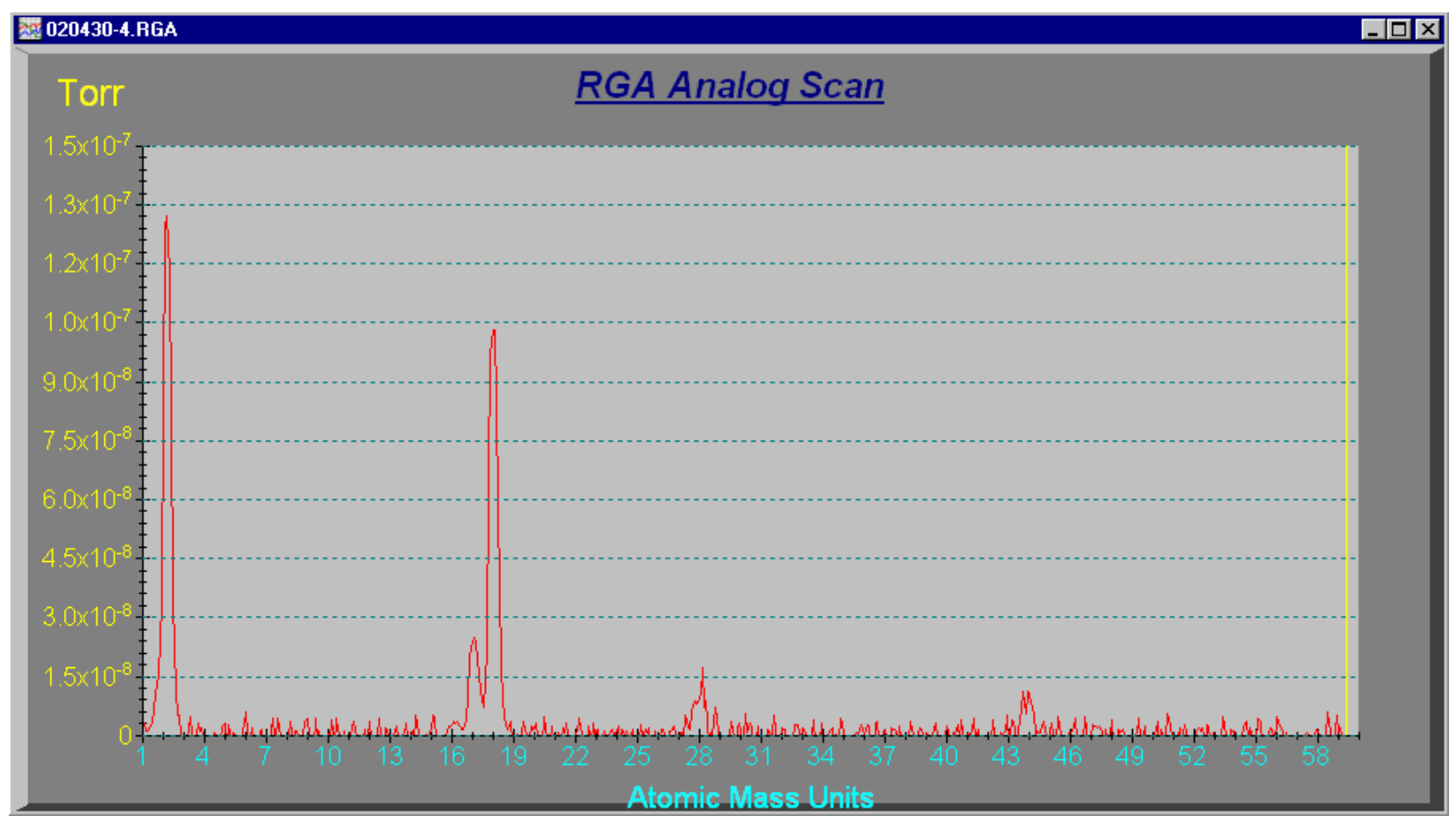

E-beam gun stabilizes 


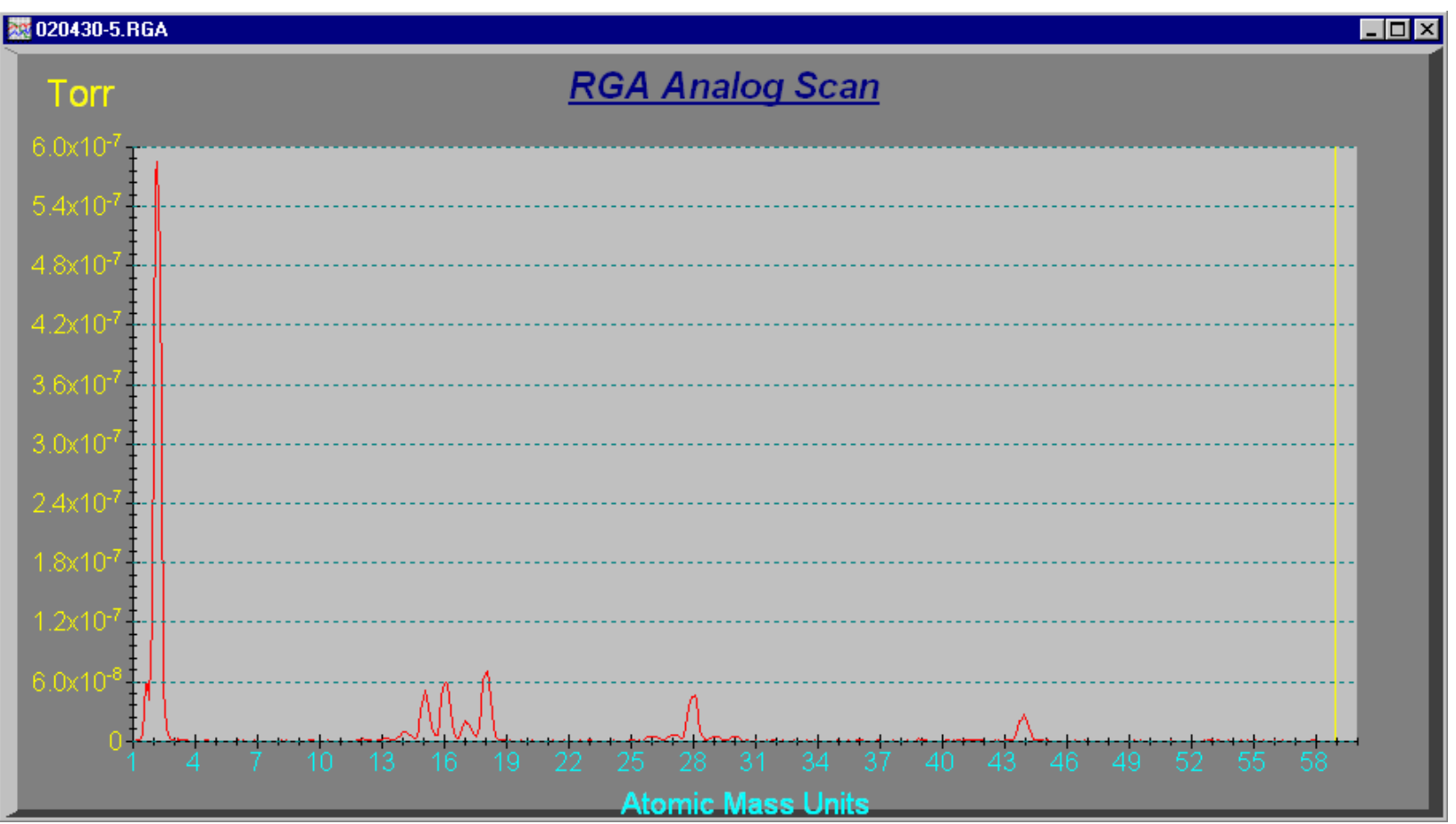

Deposition starts

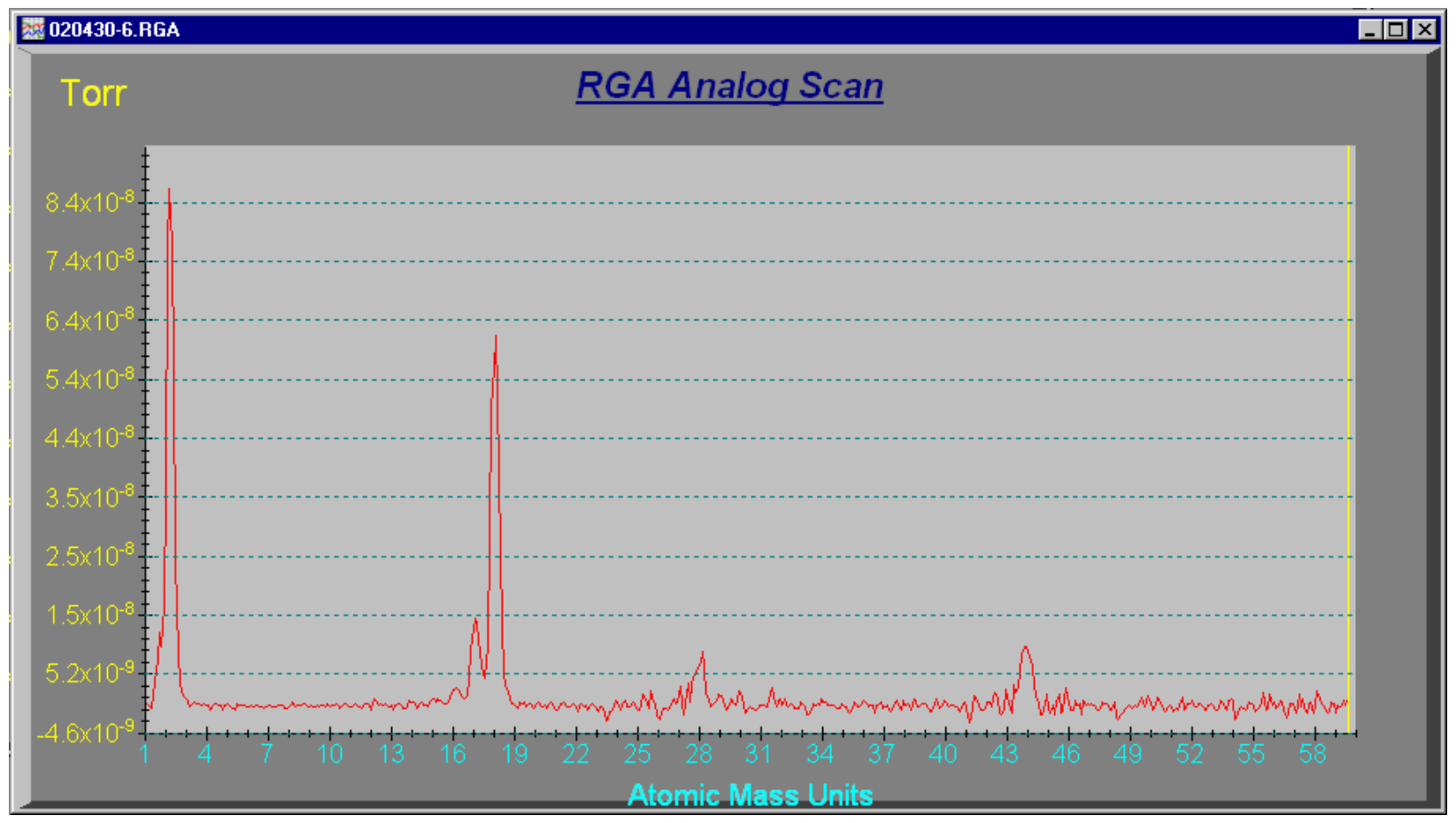

Deposition continues (Deposition almost done) 


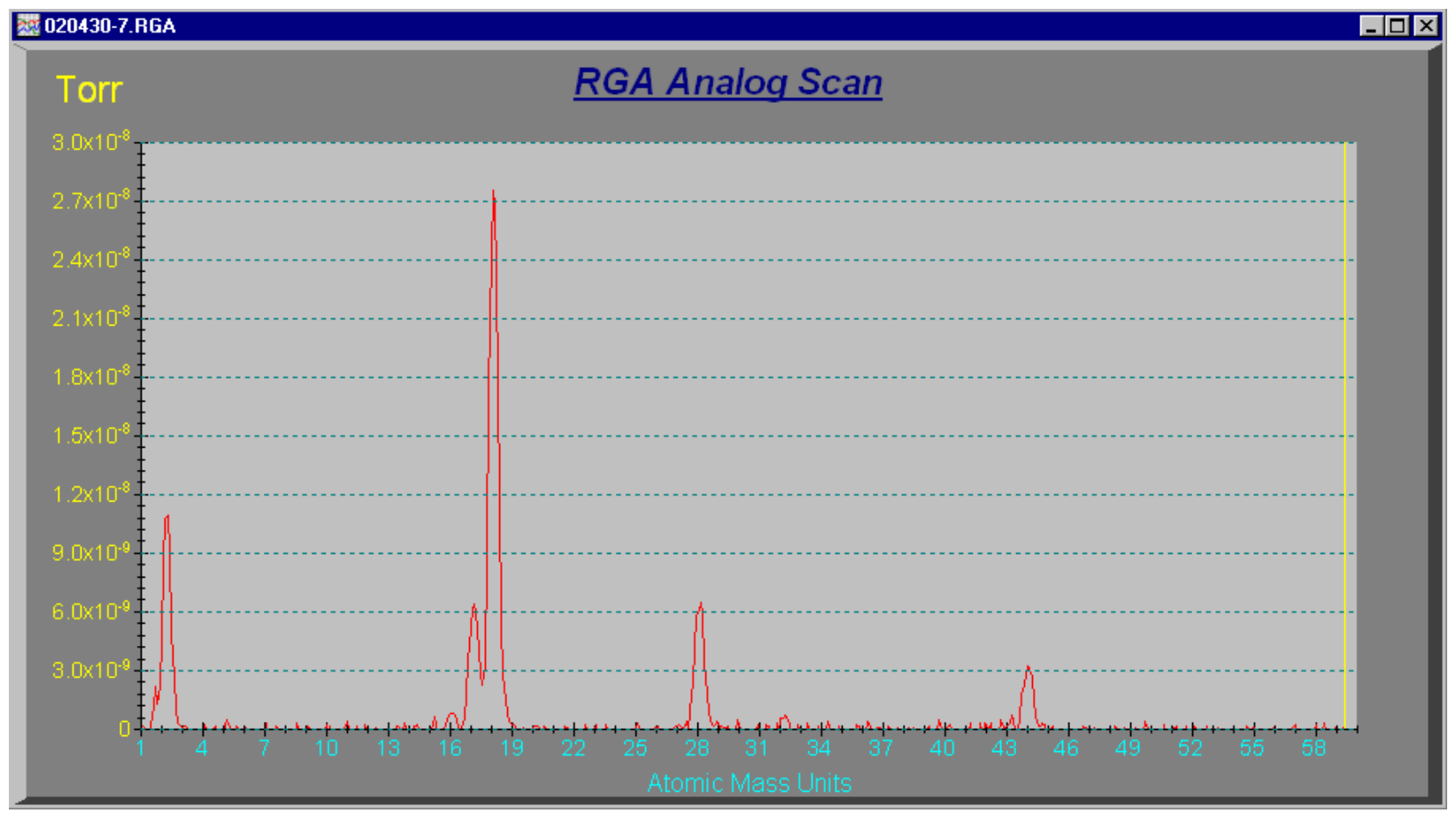

Deposition stopped 
Appendix B. The simulation code of electron movement inside the ECR RF field

\section{The electromagnetic field of circular waveguide mode TE11}

Bessel Function has the formulae:

$$
\begin{aligned}
& \frac{d}{d x}\left(x^{n} J_{n}(x)\right)=x^{n} J_{n-1}(x) \\
& n x^{n-1} \cdot J_{n}(x)+x^{n} J_{n}^{\prime}(x)=x^{n} J_{n-1}(x) \\
& J_{n}^{\prime}(x)=\frac{x^{n} J_{n-1}(x)-n x^{n-1} J_{n}(x)}{x^{n}}
\end{aligned}
$$

For $\mathrm{n}=1$, we have

$J_{1}^{\prime}(x)=\frac{x J_{0}(x)-J_{1}(x)}{x}=J_{0}(x)-\frac{J_{1}(x)}{x}$

There is also Bessel function formula:

$$
\frac{J_{1}(x)}{x}=\frac{J_{0}(x)+J_{2}(x)}{2} \stackrel{x \rightarrow 0}{\longrightarrow} 0.5 \quad \text { equation (1) }
$$

So, we know:

$J_{1}^{\prime}(0)=J_{0}(x)-\frac{J_{1}(x)}{x}=0.5$

For $\mathrm{TE}_{\mathrm{mn}}$ mode, all need a transmission factor of $e^{i h z-i \omega t}$

$$
\begin{aligned}
& H_{r}=i h K_{c} A J_{m}^{\prime}\left(K_{c} r\right) \cos (m \phi) \\
& H_{\phi}=-i h \frac{m}{r} A J_{m}\left(K_{c} r\right) \sin (m \phi) \\
& H_{z}=K_{c}^{2} A J_{m}\left(K_{c} r\right) \cos (m \phi) \\
& E_{r}=-i \omega \mu \frac{m}{r} A J_{m}\left(K_{c} r\right) \sin (m \phi) \\
& E_{\phi}=-i \omega \mu K_{c} A J_{m}^{\prime}\left(K_{c} r\right) \cos (m \phi) \\
& E_{z}=0
\end{aligned}
$$

$\mathrm{TE}_{11}$ mode:

$$
\begin{aligned}
& H_{r}=i h K_{c} A J_{1}^{\prime}\left(K_{c} r\right) \cos \phi \\
& H_{\phi}=-i h \frac{1}{r} A J_{1}\left(K_{c} r\right) \sin \phi
\end{aligned}
$$$$
H_{z}=K_{c}^{2} A J_{1}\left(K_{c} r\right) \cos \phi
$$$$
E_{r}=-i \omega \mu \frac{1}{r} A J_{1}\left(K_{c} r\right) \sin \phi
$$ 


$$
\begin{aligned}
& E_{\phi}=-i \omega \mu K_{c} A J_{1}^{\prime}\left(K_{c} r\right) \cos \phi \\
& E_{z}=0
\end{aligned}
$$

In those equations,

$h=\sqrt{k^{2}-\left(\frac{j_{11}}{b}\right)^{2}}, k=\sqrt{\mu \varepsilon} \omega, k_{c}=\frac{j_{11}}{b}, j_{11}=\left|J_{1}^{\prime}\left(j_{11}\right)=0\right|$ which equals 1.841 , (data from EM textbook). $\mathrm{b}$ is the radius of the circular waveguide.

If we pick $H_{0}=k_{c}^{2} A$, we have transmission power of TE11 mode:

$$
P_{t}=\frac{\pi b^{2}}{4}\left(\frac{\omega}{\omega_{c}}\right)^{2} \sqrt{1-\left(\frac{\omega_{c}}{\omega}\right)^{2}} \eta H_{0}^{2}\left(1-\frac{1}{k_{c}^{2} b^{2}}\right)\left[J_{1}\left(k_{c} b\right)\right]^{2}, \omega_{c}=\frac{j_{11}}{\sqrt{\mu \varepsilon} b}
$$

Also, $\sin (\phi)=y / r, \cos (\phi)=x / r$.

Once we have EM field in cylindrical system, we have them in decarte system,

$E_{x}=E_{r} \cos \phi-E_{\phi} \sin \phi$

$E_{y}=E_{r} \sin \phi+E_{\phi} \cos \phi$

$E_{z}=0 \quad$ The same for the $\mathrm{H}$ field.

For computing procedure, we have to consider the special position like $r=0$, which is in axial line.

When both $\mathrm{x}$ and $\mathrm{y}$ are zero, according to equation (1) for Bessel function,

$H_{r}=i h K_{c} A \times 0.5 \times \cos \phi$

$H_{\phi}=-i h \frac{1}{r} A J_{1}\left(K_{c} r\right) \sin \phi=-i h A \frac{K_{c}}{K_{c} r} J_{1}\left(K_{c} r\right) \sin \phi=-i h K_{c} A \times 0.5 \times \sin \phi$

$H_{z}=K_{c}^{2} A J_{1}\left(K_{c} r\right) \cos \phi=0$

$E_{r}=-i \omega \mu \frac{1}{r} A J_{1}\left(K_{c} r\right) \sin \phi=-i \omega \mu \frac{K_{c}}{K_{c} r} A J_{1}\left(K_{c} r\right) \sin \phi=-i \omega \mu A K_{c} \times 0.5 \times \sin \phi$

$E_{\phi}=-i \omega \mu K_{c} A J_{1}^{\prime}\left(K_{c} r\right) \cos \phi=-i \omega \mu A K_{c} \times 0.5 \times \cos \phi$

$E_{z}=0$

Transfer to de-carte system,

$H_{x}=i h K_{c} A \times 0.5$

$H_{y}=0$

$H_{z}=0$

$E_{x}=0$

$E_{y}=-i \omega \mu A K_{c} \times 0.5$

$E_{z}=0$

As a precaution, HFSS is used to do a dummy test for a 4.5-in diameter circular waveguide. It shows the E-field maximum at $445 \mathrm{~V} / \mathrm{m}$. My code gives about $443.9 \mathrm{~V} / \mathrm{m}$. 


\section{The computer code in $C$ language}

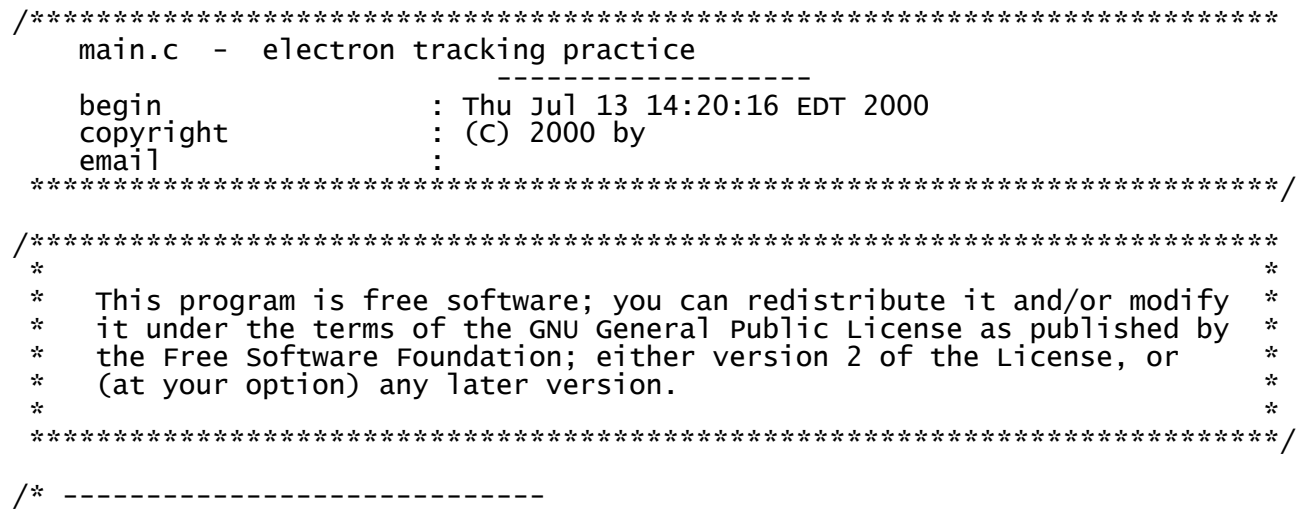

This new code applies to Circular waveguide with ECR H field, no external electric field

the system orientation : now the xy is the cross section of circular 


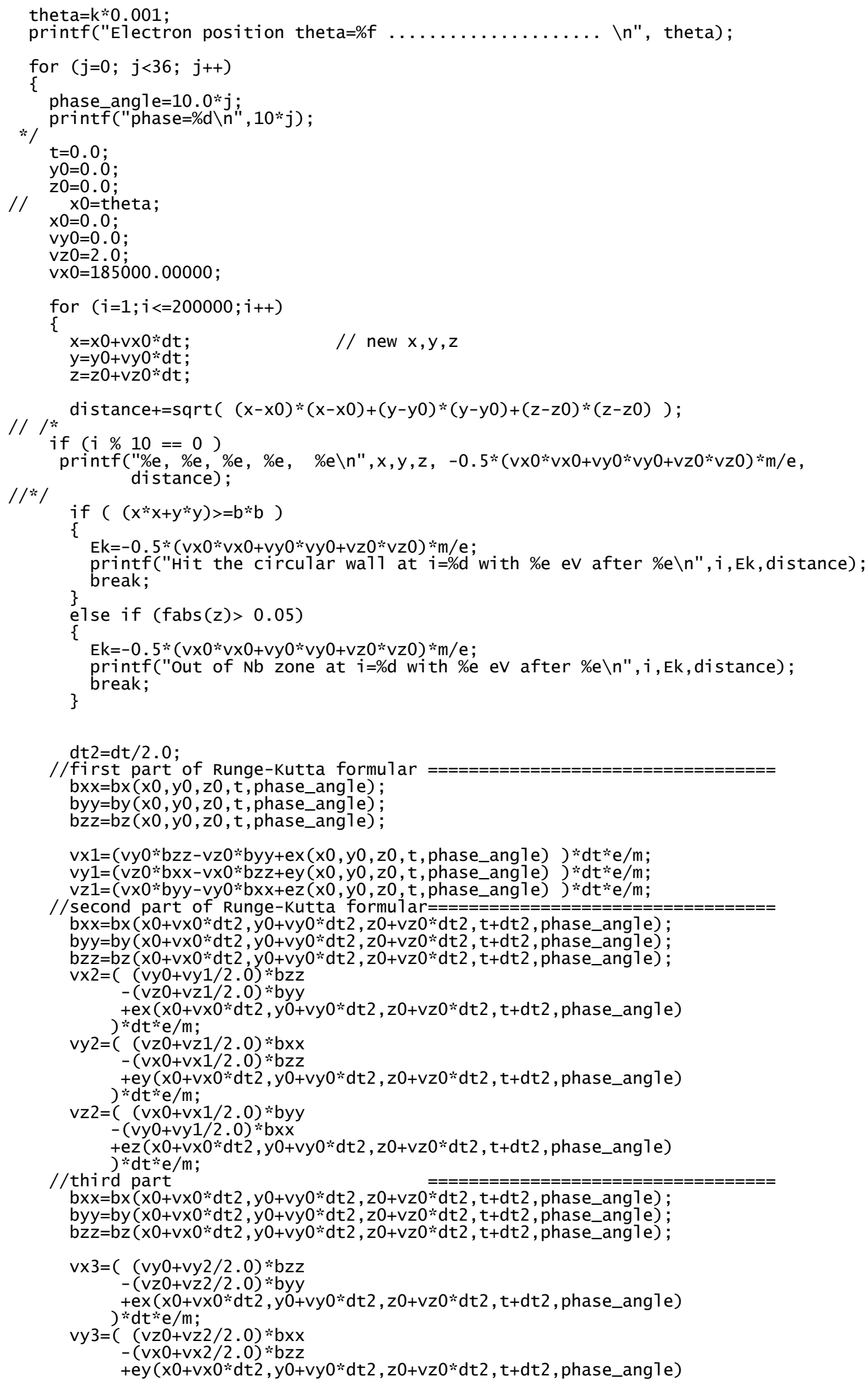




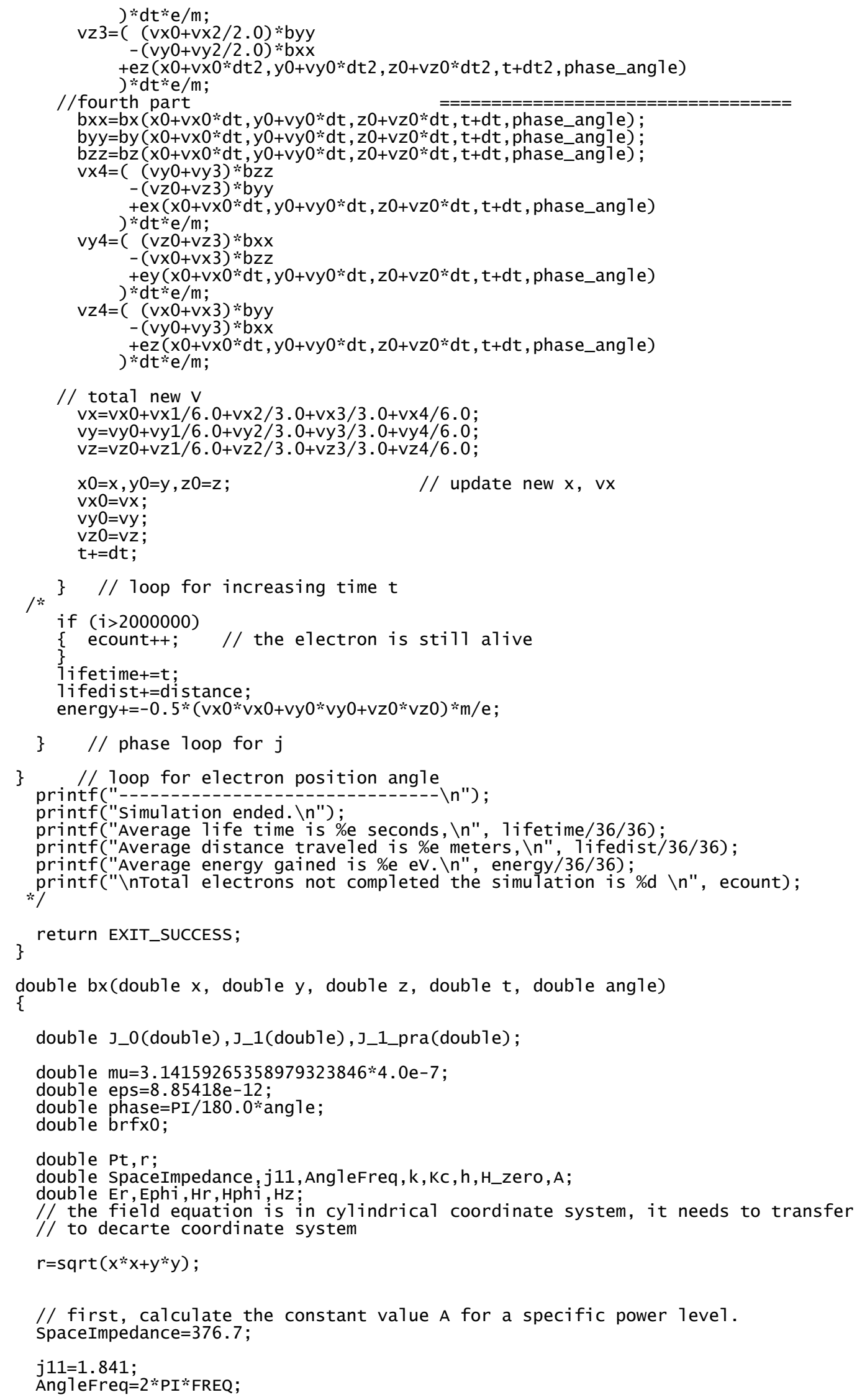




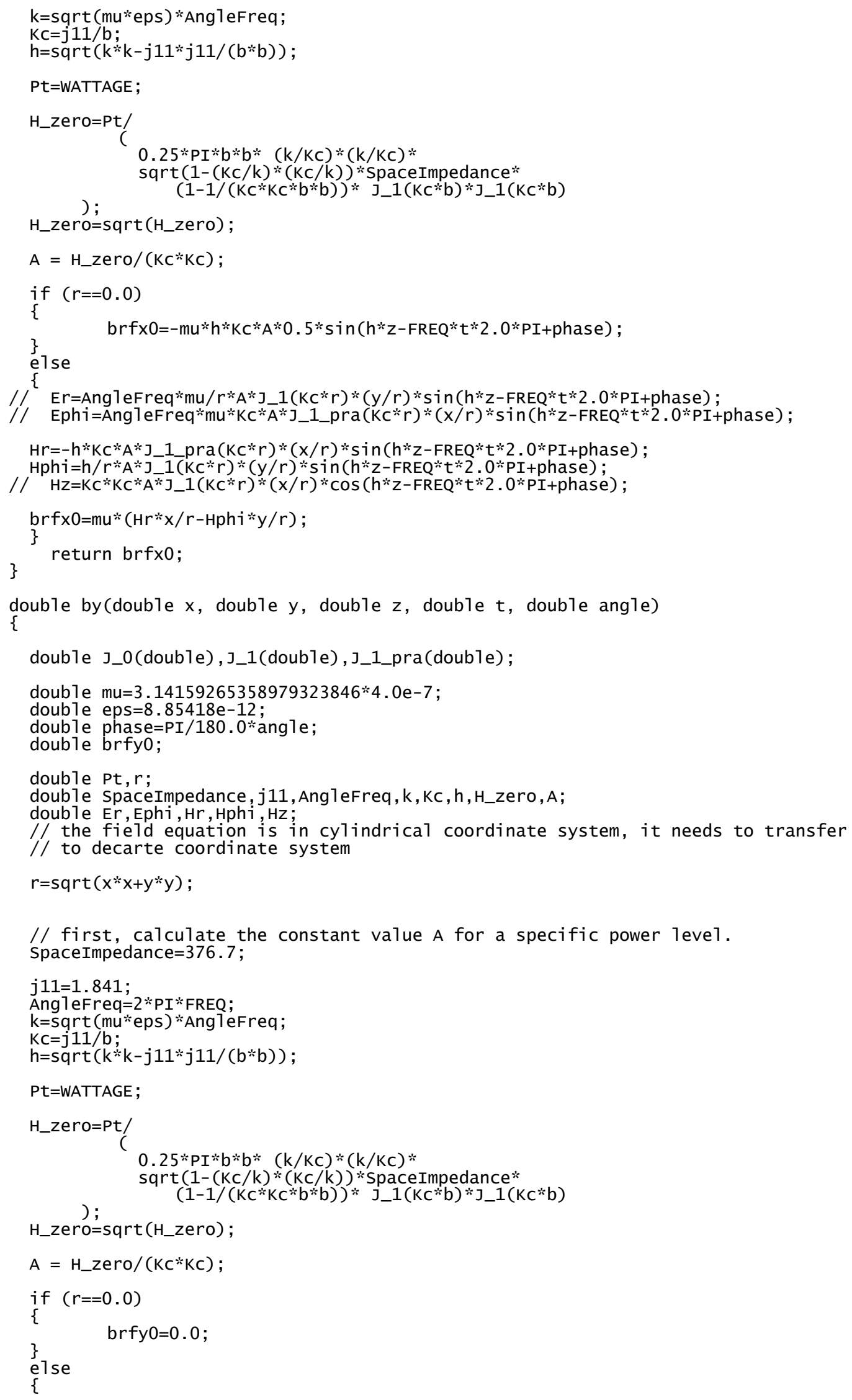


// Er=AngleFreq*mu/r*A*J_1(KC*r)*(y/r)*sin $(h * z-F R E Q * t * 2.0 * P I+$ phase $)$;

// Ephi=AngleFreq*mu*Kc*A*J_1_pra $(K C * r) *(x / r) * \sin (h * z-F R E * * * 2.0 * P I+p h a s e)$;

$H r=-h * K C * A * J \_1 \_p r a(K c * r) *(x / r) * \sin (h * z-F R E Q * t * 2.0 * P I+$ phase $)$;

Hphi=h/r*A*J_1(KC*r)*(y/r)*sin $(h * z-F R E Q * t * 2.0 * P I+$ phase $)$;

// $\mathrm{HZ}=\mathrm{KC} * K C * A * J \_1(K C * r) *(x / r) * \cos (h * Z-F R E Q * t * 2.0 * P I+p h a s e)$;

brfy $0=m u *(H r * y / r+H p h i * x / r)$;

\}

return brfy0;

double bz(double $x$, double $y$, double $z$, double $t$, double angle)

\{

double J_0(double),J_1(double),J_1_pra(double);

double $\mathrm{mu}=3.14159265358979323846 * 4.0 \mathrm{e}-7$;

double eps $=8.85418 \mathrm{e}-12$;

double phase $=P I / 180.0 *$ ang $7 e$;

double brfz0,bze=ECRFIELD;

double Pt, r;

double SpaceImpedance, j11, AngleFreq, k, Kc,h,H_zero, A;

double Er,Ephi,Hr,Hphi, Hz;

// the field equation is in cylindrical coordinate system, it needs to transfer

// to decarte coordinate system

$r=\operatorname{sqrt}\left(x^{*} x+y * y\right)$

// first, calculate the constant value A for a specific power level. SpaceImpedance $=376.7$;

$\mathrm{j} 11=1.841$;

AngleFreq $=2 * P I *$ FREQ;

$\mathrm{k}=\operatorname{sqrt}(\mathrm{mu} * \mathrm{eps}) *$ AngleFreq;

$\mathrm{KC}=\mathrm{j} 11 / \mathrm{b}$;

$h=\operatorname{sqrt}(k * k-j 11 * j 11 /(b * b))$;

Pt=WATTAGE ;

H_zero=Pt/

$0.25 * \mathrm{PI} * \mathrm{~b} * \mathrm{~b} *(\mathrm{k} / \mathrm{Kc}) *(\mathrm{k} / \mathrm{Kc}) *$

$\operatorname{sqrt}(1-(K c / k) *(K c / k)) *$ SpaceImpedance*

) ;

$(1-1 /(K c * K c * b * b)) *$ J_1 Kc*b)*J_1(Kc*b)

H_zero=sqrt (H_zero);

$A=H \_z e r o /(K C * K C)$;

if $(r==0.0)$

$\{\mathrm{brfz0}=0.0$

$/ /$ Er=AngleFreq*mu/r*A*J_1(KC*r)*(y/r)*sin (h*z-FREQ*t*2.0*PI+phase);

// Ephi=AngleFreq*mu*Kc*A*J_1_pra $(K C * r) *(x / r) * \sin (h * z-F R E * t * 2.0 * P I+p h a s e)$;

// $\mathrm{Hr}=-\mathrm{h} * \mathrm{KC} * A * J \_1 \_p r a(K C * r) *(x / r) * \sin (h * z-F R E Q * t * 2.0 * P I+$ phase $)$;

// Hphi=h/r*A*J 1(Kc*r)*(y/r)*sin $(h * z-F R E Q * t * 2.0 * P I+p h a s e)$;

$\mathrm{HZ}=\mathrm{KC} * \mathrm{KC} * \mathrm{~A} * \mathrm{~J} \_1(\mathrm{KC} * \mathrm{r}) *(\mathrm{x} / \mathrm{r}) * \cos (h * \mathrm{z}-\mathrm{FREQ} * \mathrm{t} * 2.0 * \mathrm{PI}+\mathrm{phase})$;

brfzO=mu*Hz;

\}

\}

return bze+brfz0;

double ex(double $x$, double y, double $z$, double $t$, double angle)

doub1e J_0(double),J_1(doub1e),J_1_pra(doub1e);

double mu=3.14159265358979323846*4.0e-7;

double eps $=8.85418 \mathrm{e}-12$;

double phase=PI/180.0*angle;

double Erfx0;

double Pt, r;

doub7e SpaceImpedance, $\mathrm{j} 11$, AngleFreq, $\mathrm{k}, \mathrm{Kc}, \mathrm{h}, \mathrm{H} \_z e r o, A$; 


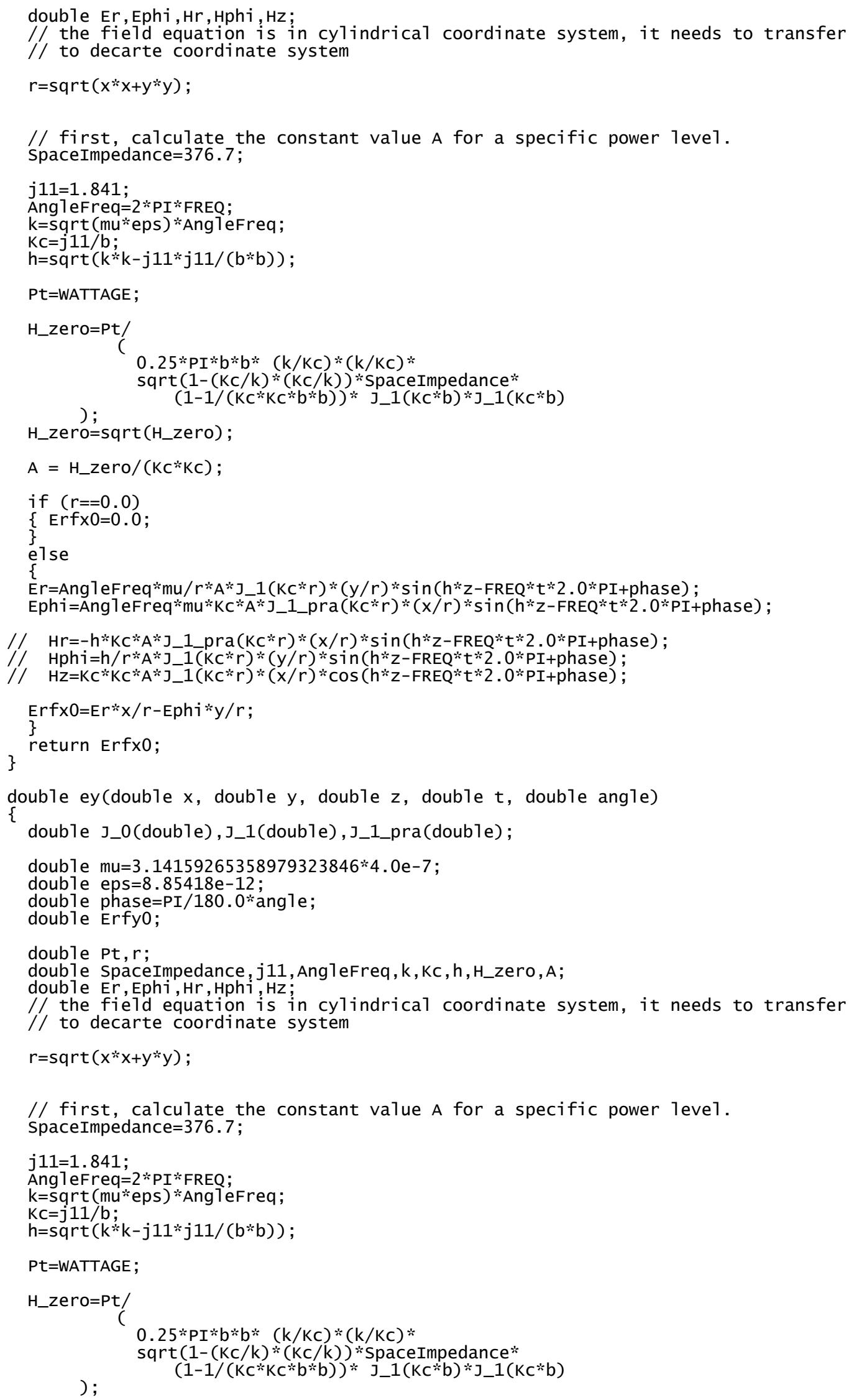




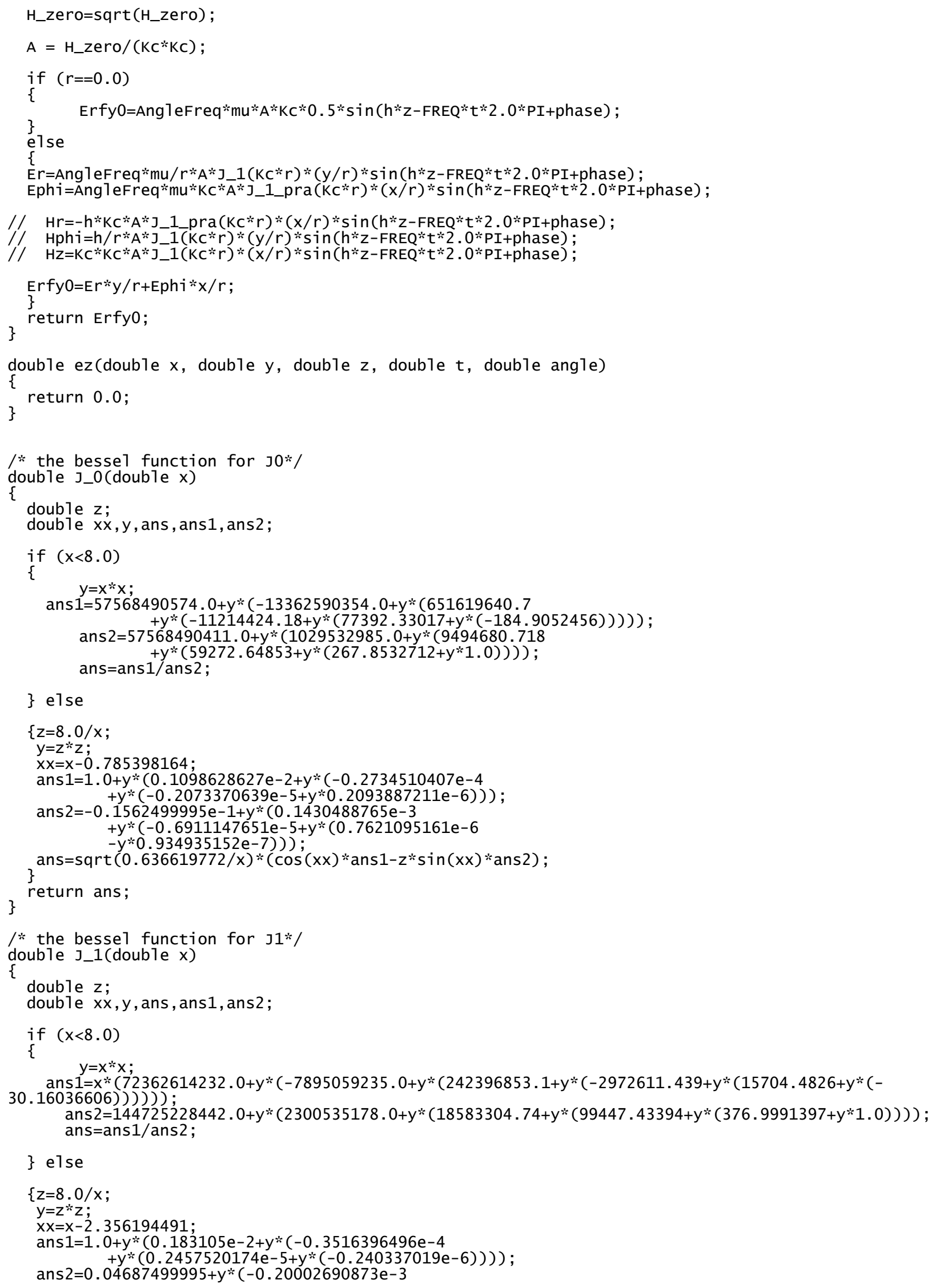


Appendix B. The simulation code of electron movement inside the ECR RF field

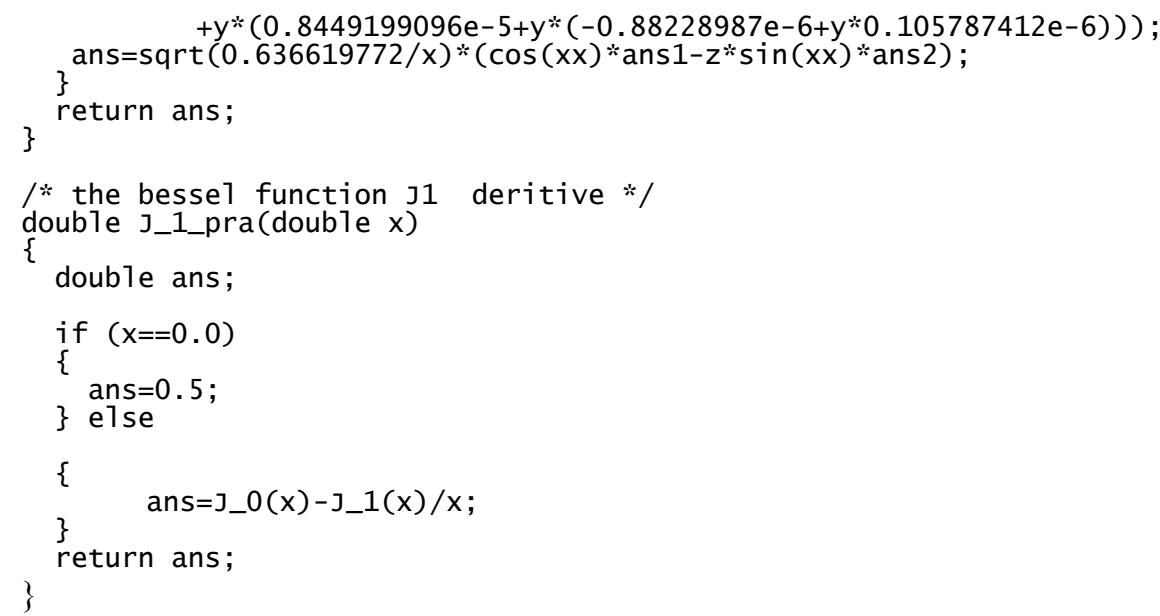




\section{Bibliography}

$1 \mathrm{~W}$. Weingarton, Progress in thin film techniques, Proc. Of $7^{\text {th }}$ workshop on RF superconductivity, Gif-sur-Yevette (1995) p.129,130

2 V. Abet-Engels, C. Benvenuti, S. Calatroni, P. Darriulat, M.A.Peck, A.-M. Valente, C.A. Van't Hof, NIM A 463 (2001)

3 H. Padamsee, et., al., RF superconductivity for Particle Accelerators, 1997

4 N. Arkani-Hamed, et., al., Physics Today, February 2002

5 G. Neil et al., Sustained Kilowatt Lasing in a Free-Electron Laser with Same-Cell Energy Recovery, Phys. Rev. Letter 84 (4), 662-665 (2000)

6 E.J.Minehara, et., al., Road Maps of the JAERI High Power FELs, Proceedings of FEL 2001 Int'l, conf., Darmstadt, Germany, 2001

7 W. Colson, et., al., Simulations of the $100 \mathrm{~kW}$ TJNAF FEL Using a Step-Tapered Undulator, Proceedings of FEL 2001 Int'l, conf., Darmstadt, Germany, 2001

8 J. Rossbach, "New Developments on Free Electron Lasers based on Self-Amplified Spontaneous Emission", PAC2001, 1-11, 2001

9 C. Wang, et., al., "Taiwan Light source", $10^{\text {th }}$ workshop on RF superconductivity, Tsukuba, Japan, 2001

10 Y. Cho, "The Evolution of SNS project", $10^{\text {th }}$ workshop on RF superconductivity, Tsukuba, Japan, 2001

11 D. Proch for the TESLA collaboration, Cost Reduction in Cavity Fabrication, $10^{\text {th }}$ workshop on RF superconductivity, Tsukuba, Japan, 2001

12 SnowMass 2001, http://www.hep.anl.gov/pvs/dpb/Snowmass.pdf, http://www.snowmass2001.org/

13 K. Shepard, Proc. Of $10^{\text {th }}$ workshop on RF superconductivity, Tsukuba, Japan, 2001

14 H. Padamsee, $10^{\text {th }}$ workshop on RF superconductivity, Tsukuba, Japan, 2001

15 P.Brown et., al., Performance of the LEP200 superconducting RF system, $9^{\text {th }}$ workshop on RF superconductivity, Santa Fe, USA, 1999

16 M. Cyrot, D. Pavuna, Introduction to Superconductivity and High-Tc Materials, World Scientific, 1999

17 L. D. Landau, E. M. Lifshitz, L. P. Pitaevskii, Statistical Physics: Part 2, 1980

18 P. G. de Gennes, Superconductivity of Metals and alloys, 1966

19 H. Padamsee, et., al., IEEE Trans. Magnetics, 19-1322, 1983

20 T. Wang, et., al., DC field emission studies, $10^{\text {th }}$ workshop on RF superconductivity, Tsukuba, Japan, 2001

21 K.M. Shirm, E. Chiaveri, B. Thony and W. Weingarten, Analysis of the coating success rate in the series production of $\mathrm{NbCu}$ superconducting RF cavities for LEP2, Proceedings of $7^{\text {th }}$ workshop on RF superconductivity, Gif-sur-Yevette (1995) p.461

22 C. Durand, W. Weingarten, P. Bosland, J. Mayer, Non Quadratic RF Losses in Niobium Sputter Coated Accelerating Structures, CERN SL/94-86 (RF), LEP2 Note 94-23

23 P. Nguyen et., al., Physical Review B 48 (1993) 6400

24 M. D. Malev, D. C. Weisser, Oxygen desorption during niobium sputtering for superconducting RF accelerators, NIM in physics research A 364 (1995), p409-415 
$25 \mathrm{~J}$. Halbritter, $10^{\text {th }}$ workshop on RF superconductivity, Tsukuba, Japan, 2001

$26 \mathrm{~W}$. Weingarten, A possible explanation of the dependence of the Q-value on the accelerating gradient for the LEP superconducting cavities, CERN-SL-96-72 RF

27 J. Knobloch et., al., Proc. Of $9^{\text {th }}$ workshop on RF superconductivity, Los Alamos, 1999

28 P. Darriulat, C. Durand, P. Janot, N. Rensing and W. Weingarten, and P. Bosland, J. Gobin and J. Martignac, Dependence of the surface resistance of Niobium coated copper cavities on the coating temperature, Proc. Of $7^{\text {th }}$ workshop on RF superconductivity, Gif-sur-Yevette (1995) p.467

29 G. Orlandi, C. Benvenuti, S. Calatroni, F. Scalambrin, Expected dependence of Nb-coated RF cavity performance on the characteristics of niobium, Proc. Of $6^{\text {th }}$ workshop on RF Superconductivity, CEBAF,USA (1993) p.718

$30 \mathrm{~J}$. A. Thornton, "Influence of apparatus geometry and deposition conditions on the structure and topography of thick sputtered coatings", JVST., Vol. 11, No. 4, (1974) p666

31 A. C. Raghuram, R. F. Bunshah, JVST Vol 9, (1972) p1389

32 G. M. Schucan, C. Benvenuti, S. Calatroni, Niobium films produced by magnetron sputtering using an Ar-He mixture as discharge gas, CERN LEP2 Note 94-23

$33 \mathrm{C}$. Benvenuti, et., al., Rare gas trapping in sputtered Nb films, Proc. Of $7^{\text {th }}$ workshop on RF superconductivity, Gif-sur-Yevette (1995) p.473

34 P.Kneisel, B.Lewis, Advanced surface cleaning methods - three years of experience with high pressure ultra-pure water rinsing of superconducting cavities, Particle Accelerator, Vol. 53, (1996), p97,110

35 V. Palmieri, REVIEW OF FABRICATION OF SC CAVITY STRUCTURES, Proceedings of Linac98, Chicago, Illinois, USA, 1998

36 M. Marino, Proc. Of $8^{\text {th }}$ workshop on RF superconductivity, Abano Terme, Italy, 1997

37 K.Geissler, etc.,al., A device for laser annealing of $\mathrm{Nb}$ films in $\mathrm{Nb}$ coated $\mathrm{Cu}$ cavities, Proc. Of $7^{\text {th }}$ workshop on RF superconductivity, Gif-sur-Yevette (1995) p.485

38 V.Palmieri, etc., al., A DC Post-magnetron configuration for niobium sputtering into $1.5 \mathrm{GHz}$ Copper monocells, Proc. Of $7^{\text {th }}$ workshop on RF superconductivity, Gif-sur-Yevette (1995) p. 485

39 R. Russo, The Report of ARCO Project, Proc. Of $10^{\text {th }}$ workshop on RF superconductivity, Tsukuba, Japan, 2001

40 J. Nagamatsu, et., al., Nature 410, 63 (2001).

41 A. Facco, et., al., SUPERCONDUCTING RF ACTIVITIES AT LNL, Proc. Of $10^{\text {th }}$ workshop on RF superconductivity, Tsukuba, Japan, 2001

42 A. Andreone, et., al., proceedings of the conference EUCAS 2001, Lyngby, Copenhagen, Denmark, 2001

43 K. Zhao, et., al., R\&D ON THE NIOBIUM-COPPER SUPERCONDUCTING CAVITY AT

PEKING UNIVERSITY, Proc. Of 9th workshop on RF superconductivity, Los Alamos, 1999

44 W. M. Holber, et., al., JVST A. Vol.11(6), 1993

45 Ansoft HFSS by Ansoft Inc., http://www.ansoft.com/

46 J. Billen et., al., Poisson/Superfish, Los Alamos, ftp://sfuser:ftpsuperfish@laacg1.lanl.gov/

47 G. Alton et., al., A High-temperature, "Volume-Type", ECR Ion Source for RIB Generation, PAC 1999, Chicago, USA

48 I. N. Bronshtein, K. A. Semendyayev, Handbook of Mathematics, Springer-Verlag 1998 
49 A.J. Lichtenberg, M.A. Lieberman, Principles of Plasma Discharges and Materials Processing, published by John Wiley and Sons, 1994

50 Y. Kim and M. Rudd, Phys. Rev. A 503594

$51 \mathrm{C}$. Bohm and J. Perrin, Retarding field analyzer for measurements of ion energy distributions and secondary electron emission coefficients in low-pressure radio frequency discharges, Review Scientific Instrumentation, 64(1), January 1993

$52 \mathrm{H}$. Padamsee, private communications

$53 \mathrm{H}$. Yakowitz et., al., A simple analytical method for thin film analysis with massive pure element standards, Proc. Of $9^{\text {th }}$ Annual SEM symposium, Chicago, Illinois, USA, 1976

54 L.N. Hand, "Our Niobium Films Contain Hydrogen”, SRF Technote, 000410-06, Cornell University, 2000

55 M. Pick, Physical Review B, Vol. 24, No. 8, p4287, 1981

56 T.Schober,H.Wenzl,Hydrogen in Metals II, vol 29 Topics in Applied Physics, SpringerVerlag 1978

57 X.-G. Zhang and W. H. Butler, Conductivity of Metallic Films and Multilayers, http://theory.ms.ornl.gov/ xgz/freekubo4/freekubo4.html

58 H. M. Rosenburg, Low Temperature Solid State Physics, Oxford University Press, 1963 


\section{Vita}

\section{Genfa Wu}

350 Adwood CT.

Hampton, VA 23605

E-mail: jeffwu2002@yahoo.com

\section{Education:}

Ph.D. of Physics, June 2002, Virginia Tech, Blacksburg, VA 24061

M.S. of Physics, June 1995, Peking University, Beijing, China

B.S. of Physics, June 1992, Peking University, Beijing, China

\section{Membership:}

Sigma Pi Sigma, Physics Honorary Society

American Physical Society 